\title{
Zero-one laws for graphs with edge probabilities decaying with distance. Part I
}

by

Saharon Shelah (Jerusalem and New Brunswick, NJ)

\begin{abstract}
Let $G_{n}$ be the random graph on $[n]=\{1, \ldots, n\}$ with the possible edge $\{i, j\}$ having probability $p_{|i-j|}=1 /|i-j|^{\alpha}$ for $j \neq i, i+1, i-1$ with $\alpha \in(0,1)$ irrational. We prove that the zero-one law (for first order logic) holds.
\end{abstract}

0. Introduction. On 0-1 laws see expository papers, e.g. Spencer [Sp]. In Euczak-Shelah [LuSh 435] the following probabilistic context was investigated. Let $\bar{p}=\left\langle p_{i}: i \in \mathbb{N}\right\rangle$ be a sequence of probabilities, i.e. real numbers in the interval $[0,1]_{\mathbb{R}}$. For each $n$ we draw a graph $G_{n, \bar{p}}$ with set of nodes $[n]:=\{1, \ldots, n\}$; for this we make the following independent drawing:

- for each (unordered) pair $\{i, j\}$ of numbers from $[n]$ we draw yes/no with probabilities $p_{|i-j|} / 1-p_{|i-j|}$, and let

$$
R_{n}=\{\{i, j\}: i, j \text { are in }[n] \text { and we draw yes }\} .
$$

We view $R_{n}$ as a symmetric irreflexive two-place relation. So we have obtained a random model $\mathcal{M}_{n, \bar{p}}^{0}=\left([n], R_{n}\right)$ (i.e. a graph), but we also consider the graph expanded by the successor relation, $\mathcal{M}_{n, \bar{p}}^{1}=\left([n], S, R_{n}\right)$, where $S=\{(l, l+1): l \in \mathbb{N}\}$ (more exactly we use $S_{n}=S \uparrow[n]$ ), and we may also consider the graph expanded by the natural order on the natural numbers, $\mathcal{M}_{n, \bar{p}}^{2}=\left([n],<, R_{n}\right)$. (We will give a little background on this structure below. But the question whether the 0-1 law holds is not discussed here.) Though we shall start dealing generally with random models, the reader can restrict himself to the case of graphs without losing comprehensibility.

In [LuSh 435] much information was obtained on when the 0-1 law holds (see Definition 1.2(1)) and when the convergence law holds (see Definition $1.2(2)$ ), depending on conditions such as $\sum_{i \in \mathbb{N}} p_{i}<\infty$ and $\sum_{i \in \mathbb{N}} i p_{i}<\infty$.

2000 Mathematics Subject Classification: 03C13, 60F20, 03C10.

Research partially supported by the United States-Israel Binational Science Foundation; publication no. 467 . 
The sequences $\bar{p}$ considered in [LuSh 435 ] were allowed to be quite chaotic, and in those circumstances the theorems were shown to be the best possible, e.g. counterexamples were obtained by replacing $\bar{p}$ by $\bar{p}^{\prime}$ where for some fast increasing sequence $\left\langle i_{k}: k \in \mathbb{N}\right\rangle$ we let

$$
p_{j}^{\prime}= \begin{cases}p_{k}, & j=i_{k} \\ 0, & (\forall k) j \neq i_{k} .\end{cases}
$$

In [Sh 463] a new version of the 0-1 law was introduced, the very weak $0-1$ law (see 1.2(3); the $h$ variant says that the difference between the probabilities for $n$ and for $m_{n}$ when $\left|n-m_{n}\right| \leq h(n)$, converges to zero), and it was proved for $\mathcal{M}_{n, \bar{p}}^{2}$ when $\sum_{i} p_{i}<\infty$ (we omit $h$ when $h(n)=1, m_{n}=n+1$ and investigate only the very weak $0-1$ law). In [Sh 548] the very weak 0-1 law was proved for models with a random two-place function and for graphs; Boppana and Spencer [BoSp] continue this by determining the best $h$ for which it holds.

Naturally arises the question what occurs if the $p_{i}$ 's are "well behaved". As in Shelah-Spencer [ShSp 304] this leads to considering $p_{i}=1 / i^{\alpha}$ (independently of $n$ ). By the results of [LuSh 435], and (essentially) [ShSp 304], the "real" cases are (for the definition of $\mathcal{M}_{n, \bar{p}}^{l}$ see above):

(A) $\mathcal{M}_{n, \bar{p}}^{0}$ where $p_{i}=1 / i^{\alpha}$ for $i>1, \alpha \in(0,1)_{\mathbb{R}}$ irrational and $p_{1}=p_{2}$,

(B) $\mathcal{M}_{n, \bar{p}}^{1}$ where $p_{i}=1 / i^{\alpha}, \alpha \in(0,1)_{\mathbb{R}}$ irrational,

(C) $\mathcal{M}_{n, \bar{p}}^{2}$ where $p_{i}=1 / i^{\alpha}, \alpha \in(1,2)_{\mathbb{R}}$.

The main aim of this work is to show that in case (A) we have the 0-1 law, also in case (B) we prove the convergence law but at present we do not know the answer to problem (C) (actually analysis indicates that the problem is whether there is a formula $\varphi(x)$ which holds in $\mathcal{M}_{n}^{2}$ for $x$ small enough and fails for $n-x, x$ small enough). Here we do not consider the linear order case. For external reasons the work is divided into two parts, the second is [Sh 517]. Note: if we let $p_{i}=1 / i^{\alpha}$ for $i \geq 1$, surely $\{l, l+1\}$ is an edge, so it is fine, but case (A) essentially becomes case (B). To preserve the distinction between $(\mathrm{A})$ and $(\mathrm{B})$ we set $p_{1}=1 / 2^{\alpha}$ in case $(\mathrm{A})$. This is one of many ways to preserve this distinction; the choice does not matter.

MaIn AND ORIGINAL CONTEXT: Random graph on $[n]$, with $p_{i}=1 / i^{\alpha}$ for $i>1$ and $p_{1}=p_{2}$; i.e. probability of the edge $\{i, j\}$ is $p_{|i-j|}$ and $\alpha \in$ $(0,1)_{\mathbb{R}} \backslash \mathbb{Q}$, i.e. is irrational.

But the proofs apply to a wider family of cases. We can make a case such that both [ShSp 304] and [LuSh 435] are particular cases: the probability for $\{i, j\}$ being an edge of $\mathcal{M}_{n}$ for $i, j \in[n]$ is $p_{i, j}^{n}$. So in [ShSp 304], $p_{i, j}^{n}=p_{n}$ and in [LuSh 435], $p_{i, j}^{n}=p_{|i-j|}$. We can consider $p_{i, j}^{n}=p_{|i-j|}^{n}$. We shall show in another paper that we shall get the same theory as in case (A) above 
in the limit, while simplifying the probabilistic arguments, if we change the context to:

SECOND CONTEXT: $\mathcal{M}_{n}$ (graph on $\{1, \ldots, n\}$ ) with probability of $\{i, j\}$ being an edge equal to

$$
p_{i, j}^{n}=\frac{1}{n^{\alpha}}+\frac{1}{2^{|i-j|}} .
$$

So the probability basically has two parts:

1) $1 / 2^{|i-j|}$ : depends only on the distance, but decays fast, so the average valency it contributes is bounded.

2) $1 / n^{\alpha}$ : does not depend on the distance, is locally negligible (i.e. for any particular $\{i, j\}$ ) but has "large integral". Its contribution to the valency of a node $i$ is on the average "huge" ( still $\ll n)$.

We can think of this as two kinds of edges. The edges of the sort $n^{-\alpha}$ are as in [ShSp 304]. The other ones still give large probability for some $i$ to have valency with no a priori bound (though not compared to $n$, e.g. $\log n$ ). In this second context the probability arguments are simpler (getting the same model theory), but we shall not deal with it here.

Note: If we look at all the intervals $[i, i+k)$, and want to get some graph there (see on $H$ below) and the probability depends only on $k$ (or at least has a lower bound $>0$ depending only on $k$ ), then the chance that for some $i$ we get this graph (by "second kind edges") is $\sim 1$; essentially this behaviour stops where $k \approx(\log n)^{b}$ for some appropriate $b>0$ (there is no real need here to calculate it). Now for any graph $H$ on $[k]$ the probability that for a particular $i<n-k$ the mapping $l \mapsto i+l$ embeds $H$ into $\mathcal{M}_{n}$ is $\geq\left(1 / k^{\alpha}\right)\left(\begin{array}{l}k \\ 2\end{array}\right)$ but is $\leq\left(1 /(k / 3)^{\alpha}\right)^{(k / 3)^{2}}$ (exactly

$$
\begin{aligned}
\prod_{\{l, m\} \in J_{1}} \frac{1}{|l-m|^{\alpha}} \cdot \prod_{\{l, m\} \in J_{2}}\left(1-\frac{1}{|l-m|^{\alpha}}\right) p_{1}^{\mid\{l:(l, l+1) \text { is an edge }\} \mid} \\
\times\left(1-p_{1}\right)^{\mid\{l:(l, l+1) \text { is not an edge }\} \mid}
\end{aligned}
$$

where $l, m \leq k$ and $J_{1}=\{\{l, m\}:(l, m)$ is an edge and $|l-m|>1\}$, $J_{2}=\{\{l, m\}:(l, m)$ is not an edge and $|l-m|>1\}$. Hence the probability that for no $i<\lfloor n / k\rfloor$ does the mapping $l \mapsto k i+l$ embed $H$ into $\mathcal{M}_{n}$ is

$$
\leq\left(1-\left(\frac{1}{k^{\alpha}}\right)^{\left(\begin{array}{c}
k \\
2
\end{array}\right)}\right)^{n / k}
$$

Hence if $\beta k^{\alpha\left(\begin{array}{l}k \\ 2\end{array}\right)}=n / k$, that is, $\beta=n / k^{\alpha\left(\begin{array}{c}k \\ 2\end{array}\right)+1}$ then this probability is $\leq e^{-\beta}$. This is because $e^{-\beta} \sim(1-\beta / n)^{n}$. We obtain

$$
\frac{k \beta}{n} \leq \frac{1}{k^{\alpha\left(\begin{array}{c}
k \\
2
\end{array}\right)} \text {. }}
$$


So the probability is small, i.e. $\beta$ large, if $k \geq\left(\frac{2}{\alpha} \log n\right)^{1 / 2}$; note that the bound for the other direction has the same order of magnitude. So with parameters, we can interpret, using a sequence of formulas $\bar{\varphi}$ and parameter $\bar{a}$, a quite long initial segment of the arithmetic (see definition below). This is very much unlike the irrational case in [ShSp 304], where a first order formula $\varphi(\bar{x})$ really says little about $\bar{x}$ : normally it says just that the $\mathrm{cl}^{k}$-closure of $\bar{x}$ is $\bar{x}$ itself or something about the few elements which are in $\mathrm{cl}^{k}(\bar{x})$ (so the first order sentences say not little about the model, but inside a model the first order formula says little). So this sounds more like the $\alpha$ rational case of [ShSp 304]. This had seemed like a sure sign of failure of the 0-1 law, but if one goes in this direction one finds it problematic to define $\bar{a}_{0}$ such that $\bar{\varphi}$ with the parameter $\bar{a}_{0}$ defines a maximal such initial segment of arithmetic, or at least find $\psi(\bar{y})$ such that for random enough $\mathcal{M}_{n}$, there is $\bar{a}_{0}$ satisfying $\psi(\bar{y})$ and if $\bar{a}_{0}$ satisfies $\psi(\bar{y})$ then $\varphi$ with this parameter defines an initial segment of arithmetic of size, say, $>\log \log \log n$. For $\bar{\varphi}$ and $\bar{a}_{0}$, to interpret an initial segment of arithmetic of size $k$ in $\mathcal{M}_{n}$ means that $\bar{\varphi}=\left\langle\varphi_{1}\left(\bar{x}^{0}, \bar{y}\right), \varphi_{2}\left(\bar{x}^{1}, \bar{y}\right), \varphi_{3}\left(\bar{x}^{2}, \bar{y}\right)\right\rangle$ is a sequence of (first order) formulas, and $\bar{a}_{0}$ is a sequence of length $\lg (\bar{y})$ such that the set $\left\{x: \mathcal{M}_{n} \models \varphi_{0}\left(x, \bar{a}_{0}\right)\right\}$ has $k$ elements, say $\left\{b_{0}, \ldots, b_{k-1}\right\}$, satisfying:

$$
\begin{aligned}
\mathcal{M}_{n}=\varphi_{1}\left(x_{0}, x_{1}, \bar{a}_{0}\right) & \Leftrightarrow \underset{l<m<k}{\bigvee}\left(x_{0}, x_{1}\right)=\left(b_{l}, b_{m}\right), \\
\mathcal{M}_{n}=\varphi_{2}\left(x_{0}, x_{1}, x_{2}, \bar{a}_{0}\right) & \Leftrightarrow \underset{l_{0}, l_{1}, l_{2}<k, l_{2}=l_{0}+l_{1}}{\bigvee}\left(x_{0}, x_{1}, x_{2}\right)=\left(b_{l_{0}}, b_{l_{1}}, b_{l_{2}}\right), \\
\mathcal{M}_{n}=\varphi_{3}\left(x_{0}, x_{1}, x_{2}, \bar{a}_{0}\right) & \Leftrightarrow \underset{l_{0}, l_{1}, l_{2}<k, l_{2}=l_{0} l_{1}}{\bigvee}\left(x_{0}, x_{1}, x_{2}\right)=\left(b_{l_{0}}, b_{l_{1}}, b_{l_{2}}\right) .
\end{aligned}
$$

But it is not a priori clear whether our first order formulas distinguish between large size and small size in such interpretation.

Note: all this does not show why the 0-1 law holds; we just explain the situation, and show we cannot prove the theory is too nice (as in [ShSp 304]) on the one hand but that this is not sufficient for failure of the 0-1 law on the other hand. Still what we say applies to both contexts, which shows that results are robust. A nice result would be if we can characterize $\left\langle p_{i}: i \in \mathbb{N}\right\rangle$ such that $\operatorname{Prob}\{i, j\}=p_{i} \Rightarrow 0-1$ holds (see below).

Our idea (to show the 0-1 law) is that though the "algebraic closure" (suitably defined) is not bounded, it is small and we can show that a first order formula $\varphi(\bar{x})$ is equivalent (in the limit case) to one "speaking" only about the algebraic closure of $\bar{x}$.

Model theoretically we do not get in the limit a first order theory which is stable and generally "low in the stability hierarchy" (see Baldwin-Shelah [BlSh 528]) for cases with probability $\sim n^{-\alpha}$ (the reason is of course that restricted to "small" formulas in some cases there is a definable linear or- 
der (or worse)). However, we get a variant of stability over a predicate: on "small" definable sets the theory is complicated, but for types with no small formulas we are in the stable situation. In fact the model theoretic setting is similar to the one in [Sh 463], but we shall not pursue this.

Note that Baldwin-Shelah [BlSh 528] deal with random models with more relations $R$ with probabilities $n^{\alpha(R)}$ (satisfying the parallel to irrationality of $\alpha$ ). There, the almost sure theory is stable. In [Sh 550] we define a family of 0-1 contexts where further drawings of relations give us a new context in this family and in all such contexts, elimination of quantifiers to the algebraic closure (as in [ShSp 304], [BlSh 528]) holds, but the context is possibly "almost nice" not nice, i.e. we allow that every $\bar{a}$ has a nontrivial closure, as in the case in which we have the successor function. Here this is dealt within the general treatment of the elimination, but not used in the main case $\mathcal{M}_{n}^{0}$. Also here we could deal with an abstract version allowing further drawing as in [Sh 550].

See more in [B196], [Sh 637].

We have chosen here a quite extreme interpretation of " $\bar{p}$ is simple, simply defined". It seems desirable to investigate the problem under more lenient conditions. A natural such family of $\bar{p}$ 's is the family of monotonic ones. Can we in this family characterize

$\left\{\bar{p}: \bar{p}\right.$ a monotone sequence, $\mathcal{M}_{n, \bar{p}}^{0}$ satisfies the $0-1$ law $\} ?$

This will be addressed and solved in [Sh 581].

The two cases considered above are protypes of some families with the 0-1 law, but there are some others, for example with the value of the exponent $\alpha$ "in the appropriate neighbourhood" of a rational (and some degenerate ones of course).

Let us review the paper.

Note: in $\S 1-\S 3$ we deal with general contexts. In these three sections sufficient conditions are proven for the $0-1$ law to hold in $0-1$ context; for notational simplicity we restrict ourselves to a vocabulary which contains finitely many predicate relations (not only a symmetric irreflexive two-place relation). The proof is based on elimination of quantifiers with the help of the closure without using probability arguments. Note that in the application we have in mind, the closure has order of magnitude up to $\sim \log \left|\mathcal{M}_{n}\right|$. In [ShSp 304] $\mathrm{cl}$ is bounded, i.e. $|\operatorname{cl}(A)|$ has a bound depending on $|A|$ (and $\alpha$ of course) only, while here it is not bounded. In the second part, $\S 4-\S 6$ deals with $\mathcal{M}_{n}^{0}$ and $\S 7$ deals with $\mathcal{M}_{n}^{1}$.

In $\S 1$ we give the basic definitions, including $A<{ }_{i} B$ (intended to mean: $B$ is the algebraic closure of $A$ but this closure has no a priori bound). The restriction to: $\mathcal{M}_{n}$ has set of elements $[n]$ (rather than some finite set) is not important for the proof. In $\S 1, A<_{i} B$ and $A<_{s} B$ are defined in terms 
of the number of embeddings of $B$ into $\mathcal{M}_{n}$ extending any embedding of $A$ into $\mathcal{M}_{n}$ in a sufficiently random model, and from $<_{i}$ we define $\mathrm{cl}^{k}(A, M)$.

In $\S 2$ a fundamental relation (i.e. given a priori) on structures $M$ is cl${ }^{k}$. From it the notions of $A<_{i} B$ and $A<_{s} B$ are defined in terms of embeddings $f \subseteq g$ of $A, B$ into a sufficiently random $\mathcal{M}_{n}$ and the relations between $g(B)$ and $\operatorname{cl}_{\mathcal{M}_{n}}^{k}\left(f(A), \mathcal{M}_{n}\right)$. Then these definitions are reconciled with those in $\S 1$, when the closure is chosen as in $\S 1$. Two axiomatic frameworks for an abstract elimination of quantifiers argument are presented. (This generalizes [BlSh 528].) These frameworks and further conditions on $\mathrm{cl}^{k}$ provide sufficient conditions for 0-1 laws and convergence laws.

Note: in $\S 2$ we retain using "relation free amalgamation" (as in [BlSh 528], but in [Sh 550] we will use a more general one). However we waive "random $A$ has no nontrivial closure", hence use "almost nice" rather than nice (and also waive the a priori bounds on closure).

In $\S 3$ we deal with the case where the natural elimination of quantifiers is to monadic logic. This seems natural, although it is not used later.

We now proceed to describe part II. The main point of $\S 4$ is to introduce a notion of weight $\mathbf{w}(A, B, \lambda)$ which depends on an equivalence relation $\lambda$ on $B \backslash A$. (Eventually such $\lambda$ will be defined in terms of the "closeness" of images of points in $B$ under embeddings into $\mathcal{M}_{n}$.) Relations $A \leq_{i}^{*} B$ and $A \leq_{s}^{*} B$ are defined in terms of $\mathbf{w}$. The intention is that $\leq_{i}^{*}$ is $\leq_{i}$ etc., thus we will have a direct characterization of the latter.

$\S 5$ contains the major probability estimates. The appropriate $\lambda$ is defined and thus the interpretations of $<_{i}^{*}$ and $<_{s}^{*}$ in the first context $\left(\mathcal{M}_{n}^{0}, p_{i}=\right.$ $1 / i^{\alpha}$ for $i>1$ ). Several proofs are analogous to those in [ShSp 304] and [BlSh 528], so we treat them only briefly. The new point is the dependence on distance, and hence the equivalence relations $\lambda$.

In $\S 6$ it is shown that the $<_{i}^{*}$ and $<_{s}^{*}$ of $\S 5$ agree with the $<_{i}$ and $<_{s}$ of $\S 1$. Further, if $\mathrm{cl}^{k}$ is defined from the weight function in $\S 4$, these agree with $<_{i},<_{s}$ as in $\S 2$ and we prove the "simple almost niceness" of Definition 2.13, so the "elimination of quantifiers modulo quantification on (our) algebraic closure" result applies. This completes the proof of the $0-1$ law for the first context. The model theoretic considerations in the proof of this version of niceness (e.g. compactness) have been less easy than I expected.

$\S 7$ deals with the changes needed for $\mathcal{M}_{n, \bar{p}}^{1}$ where only the convergence law is proved.

Note: our choice " $\mathcal{M}_{n}$ has set of elements $[n]$ " is just for simplicity (and tradition), we could have $\mathcal{M}_{n}$ with set of elements a finite set (not even fixed) and replace $n^{\varepsilon}$ by $\left\|\mathcal{M}_{n}\right\|^{\varepsilon}$ as long as "for each $k$ for every random enough $\mathcal{M}_{n}$ we have $\left\|\mathcal{M}_{n}\right\|>k$ ". Also the choice of $n^{\varepsilon}$ in Definition 1.4 is the most natural but not unique. The paper is essentially self contained, assuming only basic knowledge of first order logics and probability. 
Notation 0.1 . $\mathbb{N}$ is the set of natural numbers $(\{0,1,2, \ldots\})$.

- $\mathbb{R}$ is the set of reals.

- $\mathbb{Q}$ is the set of rationals.

- $i, j, k, l, m, n, r, s, t$ are natural numbers.

- $p, q$ are probabilities.

- $\alpha, \beta, \gamma, \delta$ are reals.

- $\varepsilon, \zeta, \xi$ are positive reals (usually quite small) and also $\mathbf{c}$ (for constant in inequalities).

- $\lambda$ is an equivalence relation.

- $M, N, A, B, C, D$ are graphs or more generally models (that is, structures, finite of fixed finite vocabulary, for notational simplicity with predicates only, if not said otherwise; the reader can restrict himself to graphs).

- $|M|$ is the set of nodes or elements of $M$, so $\|M\|$ is the number of elements.

- $\mathcal{M}$ denotes a random model.

- $\mu$ denotes a distribution (in the probability sense).

- $[n]$ is $\{1, \ldots, n\}$.

- $A \subseteq B$ means $A$ is a submodel of $B$, i.e. $A$ is $B$ restricted to the set of elements of $A$ (for graphs: induced subgraph). We shall not always distinguish strictly between a model and its set of elements. If $X$ is a set of elements of $M$, then $M \uparrow X$ is $M$ restricted to $X$.

- $a, b, c, d$ are nodes of graphs / elements of models.

- $\bar{a}, \bar{b}, \bar{c}, \bar{d}$ are finite sequences of nodes/elements.

- $x, y, z$ are variables.

- $\bar{x}, \bar{y}, \bar{z}$ are finite sequences of variables.

- $X, Y, Z$ are sets of elements.

- $\tau$ is a vocabulary for simplicity with predicates only (we may restrict a predicate to being symmetric and/or irreflexive (as for graphs)).

- $\mathcal{K}$ is a family of models of fixed vocabulary, usually $\tau=\tau_{\mathcal{K}}$.

- The vocabulary of a model $M$ is $\tau_{M}$.

- $\bar{a}^{\wedge} \bar{b}$ or $\bar{a} \bar{b}$ is the concatenation of the two sequences, $\bar{a}^{\wedge} b$ or $\bar{a} b$ is $\bar{a}^{\wedge}\langle b\rangle$.

- The extensions $g_{1}, g_{2}$ of $f$ are disjoint if $x_{1} \in \operatorname{dom}\left(g_{1}\right) \backslash \operatorname{dom}(f)$, $x_{2} \in \operatorname{dom}\left(g_{2}\right) \Rightarrow x_{1} \neq x_{2}$.

Acknowledgements. We thank John Baldwin and Shmuel Lifsches and Çiğdem Gencer and Alon Siton for helping in various ways and stages to make the paper more user-friendly.

1. Weakly nice classes. We interpret here "few" by: "for each $\varepsilon$ for every random enough $\mathcal{M}_{n}$, there are (for each parameter) $<n^{\varepsilon}$ ". We could use other functions as well. 
General Context 1.1. (i) Let $\tau$ be a fixed vocabulary which for simplicity is finite and has only predicates, i.e. symbols for relations.

(ii) Let $\mathcal{K}$ be a class of finite $\tau$-models closed under isomorphism and submodels. For $n \in \mathbb{N}, \mathcal{K}_{n}$ is a set of $\tau$-models which usually have universe $[n]=\{1, \ldots, n\}$ (just for notational simplicity).

(iii) Let $\mathcal{M}_{n}$ be a random model in a fixed vocabulary $\tau$ which is an element of $\mathcal{K}_{n}$, that is, we have a function $\mu_{n}: \mathcal{K}_{n} \rightarrow[0,1]_{\mathbb{R}}$ such that $\sum\left\{\mu_{n}(M): M \in \mathcal{K}_{n}\right\}=1$. Then $\mu_{n}$ is called a distribution and $\mathcal{M}_{n}$ the random model for $\mu_{n}$; we restrict ourselves to finite or countable $\mathcal{K}_{n}$. We omit $\mu_{n}$ when clear from the context.

(iv) We call $\left(\mathcal{K},\left\langle\left(\mathcal{K}_{n}, \mu_{n}\right): n<\omega\right\rangle\right)$ a $0-1$ context and denote it by $\mathfrak{K}$ and usually consider it fixed; we may "forget" to mention $\mathcal{K}$. So,

(v) The probability of $\mathcal{M}_{n} \models \varphi$ is

$$
\operatorname{Prob}\left(\mathcal{M}_{n} \models \varphi\right)=\sum\left\{\mu_{n}(M): M \in \mathcal{K}_{n}, M \models \varphi\right\} .
$$

(vi) The meaning of "for every random enough $\mathcal{M}_{n}$ we have $\Psi$ " is

$$
\left\langle\operatorname{Prob}\left(\mathcal{M}_{n}=\Psi\right): n\langle\omega\rangle \text { converges to } 1 ;\right.
$$

alternatively, we may write "almost surely $\mathcal{M}_{n} \models \Psi$ ".

Definition 1.2. (1) The $0-1$ law (for $\mathfrak{K}$ ) says: whenever $\varphi$ is a f.o. (= first order) sentence in vocabulary $\tau$,

$$
\left\langle\operatorname{Prob}\left(\mathcal{M}_{n} \models \varphi\right): n<\omega\right\rangle \text { converges to } 0 \text { or to } 1 .
$$

(2) The convergence law says: whenever $\varphi$ is a f.o. sentence in $\tau$,

$$
\left\langle\operatorname{Prob}\left(\mathcal{M}_{n}=\varphi\right): n\langle\omega\rangle\right. \text { is a convergent sequence. }
$$

(3) The very weak 0-1 law says: whenever $\varphi$ is a f.o. sentence in $\tau$,

$$
\lim _{n}\left[\operatorname{Prob}\left(\mathcal{M}_{n+1}=\varphi\right)-\operatorname{Prob}\left(\mathcal{M}_{n} \models \varphi\right)\right]=0 .
$$

(4) The $h$-very weak 0-1 law for $h: \mathbb{N} \rightarrow \mathbb{N} \backslash\{0\}$ says: whenever $\varphi$ is a f.o. sentence in $\tau$,

$$
0=\lim _{n} \max _{l, k \in[0, h(n)]}\left|\operatorname{Prob}\left(\mathcal{M}_{n+k}=\varphi\right)-\operatorname{Prob}\left(\mathcal{M}_{n+l}=\varphi\right)\right|
$$

Notation 1.3. $f: A \hookrightarrow B$ means: $f$ is an embedding of $A$ into $B$ (in the model theoretic sense, for graphs: isomorphism onto the induced subgraph).

Definition 1.4. (1) Let

$$
\mathcal{K}_{\infty}=\left\{A: A \text { is a finite } \tau \text {-model, } 0<\limsup _{n}\left[\operatorname{Prob}\left((\exists f)\left(f: A \hookrightarrow \mathcal{M}_{n}\right)\right)\right]\right\} .
$$

Recall $(1.1(\mathrm{v}))$ that $\operatorname{Prob}\left((\exists f)\left(f: A \hookrightarrow \mathcal{M}_{n}\right)\right)=\sum\left\{\mu_{n}\left(\mathcal{M}_{n}\right): \mathcal{M} \in \mathcal{K}_{n}\right.$ and there is an embedding $\left.f: A \hookrightarrow \mathcal{M}_{n}\right\}, n<\omega$.

Also let $T_{\infty}:=\{\varphi: \varphi$ is a f.o. sentence in the vocabulary of $\mathcal{K}$ such that every random enough $\mathcal{M}_{n}$ satisfies it $\}$. 
(2) $A \leq B$ means: $A, B \in \mathcal{K}_{\infty}$ and $A$ is a submodel of $B$; of course $A<B$ means $A \leq B$ and $A \neq B$, and similarly for others below.

(3) $A \leq_{i} B$ means: $A \leq B$ and for each $\varepsilon \in \mathbb{R}^{+}$we have:

$$
1=\lim _{n}\left[\operatorname{Prob}\left(\begin{array}{l}
\text { if } f_{0}: A \hookrightarrow \mathcal{M}_{n} \\
\text { then the number of } f_{1} \text { satisfying } \\
f_{0} \subseteq f_{1}: B \hookrightarrow \mathcal{M}_{n} \text { is } \leq n^{\varepsilon} .
\end{array}\right)\right] .
$$

Also let $\operatorname{ex}\left(f_{0}, B, M\right)=\operatorname{ex}\left(f_{0}, A, B, M\right):=\{f: f$ is an embedding of $B$ into $M$ extending $\left.f_{0}\right\}$.

(4) $A \leq_{s} B$ means: $A \leq B$ and there is no $C$ with $A<_{i} C \leq B$.

(5) $A<_{p r} B$ means: $A<_{s} B$ and there is no $C$ with $A<_{s} C<_{s} B$ (pr abbreviates primitive).

(6) $A<_{a} B$ means: $A \leq B$ and, for some $\varepsilon \in \mathbb{R}^{+}$for every random enough $\mathcal{M}_{n}$, for no $f: A \hookrightarrow \mathcal{M}_{n}$ do we have $n^{\varepsilon}$ pairwise disjoint extensions $g$ of $f$ satisfying $g: B \hookrightarrow \mathcal{M}_{n}$.

(7) $A \leq_{m}^{s} B$ means: $A \subseteq B$ are from $\mathcal{K}$ and for every $X \subseteq B$ with $\leq m$ elements, we have $A \uparrow(A \cap X) \leq_{s} B\lceil X$.

(8) $A \leq_{k, m}^{i} B$ means: $A \subseteq B$ are from $\mathcal{K}$ and for every $X \subseteq B$ with $\leq k$ elements there is $Y$ with $X \subseteq Y \subseteq B$ and with $\leq m$ elements such that $A\left\lceil(A \cap Y) \leq_{i} B\lceil Y\right.$.

(9) For $h: \mathbb{N} \times \mathbb{R}^{+} \rightarrow \mathbb{R}^{+}$, we define $A \leq_{i}^{h} B$ as in part (3) replacing $n^{\varepsilon}$ by $h(n, \varepsilon)$, and similarly $A \leq_{a}^{h} B$ (in part (6)), hence $A \leq_{s}^{h} B, A \leq_{p r}^{h} B$, $A<_{a}^{h} B, A \leq_{m}^{s, h} B, A \leq_{k, m}^{i, h} B$.

REMARK 1.5. (1) In these circumstances the original notion of algebraic closure is not well behaved. $A \leq_{i} B$ provides a reasonable substitute for $A \subseteq B \subseteq \operatorname{acl}(A)$.

(2) Note: for $\leq_{i}^{h}$ to be transitive we need: for every $\varepsilon_{1}>0$ for some $\varepsilon_{2}>0$ for every $n$ large enough $h\left(n, \varepsilon_{2}\right) \cdot h\left(n, \varepsilon_{2}\right) \leq h\left(n, \varepsilon_{1}\right)$.

(3) Why do we restrict ourselves to $\mathcal{K}_{\infty}$ (in 1.4(1)-(6))? The relations in 1.4(1)-(6) describe the situation in the limit. So why in 1.4(7), (8) we do not restrict ourselves to $A, B \in \mathcal{K}_{\infty}$ ? Because for $A \in \mathcal{K}_{\infty}$, for quite random $\mathcal{M}_{n}$, and $f: A \hookrightarrow \mathcal{M}_{n}$ the set $\mathrm{cl}^{k}\left(f(A), \mathcal{M}_{n}\right)$ may be quite large, say with $\log n$ elements, so it (more exactly the restriction of $\mathcal{M}_{n}$ to it) is not necessarily in $\mathcal{K}_{\infty}$; this is a major point here.

Let us expand. If $A \in \mathcal{K}$ has a copy in a random enough $\mathcal{M}_{n}$ and we have the 0-1 law then $T_{\infty}$ (see 1.4(1)) says that copies of $A$ occur. But if $\mathcal{M}_{n}$ is random enough, and for example $A=\left\{a_{1}, a_{2}, a_{3}\right\} \leq \mathcal{M}_{n}$, and $B=\mathcal{M}_{n}\left\lceil\mathrm{cl}^{k}\left(\left\{a_{1}, a_{2}, a_{3}\right\}, \mathcal{M}_{n}\right)\right.$ has, say, $\log n$ elements then it does not follow that $T_{\infty}=$ "a copy of $B$ occurs", as $\mathcal{M}_{n}$ may not be random enough 
for $B$. Still for statements like

$$
\left(\exists x_{1}, x_{2}, x_{3}\right)\left(\mathrm{cl}^{k}\left(\left\{x_{1}, x_{2}, x_{3}\right\}\right) \models \varphi\right)
$$

the model $\mathcal{M}_{n}$ may be random enough. The point is that the size of $B$ could be computed only after we have $\mathcal{M}_{n}$.

Another way to look at it: models $M_{\infty}$ of $T_{\infty}$ are very random in a sense, but $\operatorname{cl}\left(\left\{a_{1}, a_{2}, a_{3}\right\}, M_{\infty}\right)$ is infinite, may even be uncountable, so randomness concerning it becomes meaningless.

Definition 1.6. For $A \subseteq M$ and $k<\omega$ define:

(a) $\operatorname{cl}^{k}(A, M)=\bigcup\left\{B: B \subseteq M, B \cap A \leq_{i} B\right.$, and $\left.|B| \leq k\right\}$,

(b) $\mathrm{cl}^{k, 0}(A, M)=A$,

(c) $\mathrm{cl}^{k, m+1}(A, M)=\mathrm{cl}^{k}\left(\mathrm{cl}^{k, m}(A, M), M\right)$.

Observation 1.7. (1) For all $l, k \in \mathbb{N}$ and $\varepsilon \in \mathbb{R}^{+}$we have

$$
1=\lim _{n}\left[\operatorname{Prob}\left(A \subseteq \mathcal{M}_{n},|A| \leq l \Rightarrow\left|\mathrm{cl}^{k}\left(A, \mathcal{M}_{n}\right)\right|<n^{\varepsilon}\right)\right] .
$$

(2) Moreover, for every $k \in \mathbb{N}$ and $\varepsilon \in \mathbb{R}^{+}$for some $\zeta \in \mathbb{R}^{+}$(actually, any $\zeta<\varepsilon /(k+1)$ will do $)$ we have

$$
1=\lim _{n}\left[\operatorname{Prob}\left(|A| \leq \mathcal{M}_{n},|A| \leq n^{\zeta} \Rightarrow\left|\mathrm{cl}^{k}\left(A, \mathcal{M}_{n}\right)\right|<n^{\varepsilon}\right)\right] .
$$

Remark 1.8. True for $\mathrm{cl}^{k, m}$ too, but we can use Claim 1.16 instead.

Definition 1.9. $\mathfrak{K}=\left\langle\mathcal{M}_{n}: n\langle\omega\rangle\right.$ is weakly nice if whenever $A<_{s} B$ (so $A \neq B$ ), there is $\varepsilon \in \mathbb{R}^{+}$with

$$
1=\lim _{n}\left[\operatorname{Prob}\left(\begin{array}{l}
\text { if } f_{0}: A \hookrightarrow \mathcal{M}_{n} \text { then there is } \mathcal{F} \text { with }|\mathcal{F}| \geq n^{\varepsilon} \text { and } \\
\text { (i) } f \in \mathcal{F} \Rightarrow f_{0} \subseteq f: B \hookrightarrow \mathcal{M}_{n}, \\
\text { (ii) } f^{\prime} \neq f^{\prime \prime} \in \mathcal{F} \Rightarrow \operatorname{Rang}\left(f^{\prime}\right) \cap \operatorname{Rang}\left(f^{\prime \prime}\right)=\operatorname{Rang}\left(f_{0}\right)
\end{array}\right)\right] \text {. }
$$

If clause (ii) holds we say the $f \in \mathcal{F}$ are pairwise disjoint over $f_{0}$ or over $A$. In such circumstances we say that $\varepsilon$ witnesses $A<_{s} B$.

REMARK 1.10. Being weakly nice means there is a gap between being pseudo-algebraic and nonpseudo-algebraic (both in our sense), so we have a strong dichotomy.

FACT 1.11. For $A, B, C$ in $\mathcal{K}_{\infty}$ :

(1) $A \leq_{i} A$,

(2) $A \leq_{i} B, B \leq_{i} C \Rightarrow A \leq_{i} C$,

(3) $A \leq_{s} A$,

(4) if $A_{1} \leq B_{1}, A_{2} \leq B_{2}, A_{1} \leq A_{2}, B_{1} \leq B_{2}, B_{1} \backslash A_{1}=B_{2} \backslash A_{2}$ then $A_{2} \leq_{s} B_{2} \Rightarrow A_{1} \leq_{s} B_{1}$ and $A_{1} \leq_{i} B_{1} \Rightarrow A_{2} \leq_{i} B_{2}$,

(5) $A<_{i} B$ iff for every $C$ we have $A \leq C<B \Rightarrow C<_{a} B$

(6) if $A \leq C$ then for some $B, A \leq_{i} B \leq_{s} C$; in fact, it is unique. 
Proof. Easy (e.g. 1.11(5) by the $\Delta$-system argument (for fixed size of the sets and many of them); note $|B|$ is constant). $\mathbf{m}_{1.11}$

Claim 1.12. If $A<_{s} B<_{s} C$ then $A<_{s} C$.

First proof. If not, then for some $B^{\prime}$ we have $A<_{i} B^{\prime} \leq C$. If $B^{\prime} \subseteq B$ we get a contradiction to $A<_{s} B$, so assume $B^{\prime} \nsubseteq B$. By 1.13(1) below we have $B^{\prime} \cap B<_{i} B^{\prime}$ so by $1.11(4)$ we have $B<_{i} B \cup B^{\prime}$, hence we get a contradiction to $B<_{s} C$.

Second proof. (Assuming $\mathfrak{K}$ is weakly nice, i.e. if we define $<_{s}$ by 1.9.) Let $\varepsilon>0$ witness $A<_{s} B$ in Definition 1.9 and let $\zeta>0$ witness $B<_{s} C$ in Definition 1.9. Choose $\xi=\min \{\varepsilon / 2, \zeta / 2\}$ (actually just $\xi<\varepsilon \wedge \xi<\zeta$ suffices). Let $n$ be large enough, in particular $n^{\varepsilon}>|C|$, and let $f_{0}: A \hookrightarrow \mathcal{M}_{n}$. So we have (almost surely) $\left\{f_{1}^{i}: i<i^{*}\right\}$, where $i^{*} \geq n^{\varepsilon}$, and $f_{0} \subseteq f_{1}^{i}$ and $f_{1}^{i}: B \hookrightarrow \mathcal{M}_{n}$ and the $f_{1}^{i}$ 's are pairwise disjoint over $A$.

Now, almost surely for every $i$ we have $\left\{f_{2}^{i, j}: j<j_{i}^{*}\right\}$ with $f_{1}^{i} \subseteq f_{2}^{i, j}$ and $f_{2}^{i, j}: C \hookrightarrow \mathcal{M}_{n}$ and, fixing $i$, the $f_{2}^{i, j}$ 's are pairwise disjoint over $B$ and $j_{i}^{*} \geq n^{\zeta}$.

Clearly (when the above holds) for $l^{*}=n^{\xi}$ we can find $\left\{j_{k}: k \leq l^{*}\right\}$ such that $\left\{f_{2}^{k, j_{k}}: k<l^{*}\right\}$ are pairwise disjoint over $A$ : just choose $j_{k}$ by induction on $k$ such that $\operatorname{Rang}\left(f_{2}^{k, j_{k}} \uparrow(C \backslash B)\right)$ is disjoint from

$$
\bigcup\left\{\operatorname{Rang}\left(f_{1}^{i} \uparrow(B \backslash A)\right): i<l^{*}\right\} \cup \bigcup\left\{\operatorname{Rang}\left(f_{2}^{i, j_{i}} \uparrow(C \backslash B)\right): i<k\right\} ;
$$

at stage $k$, the number of inappropriate $j<n^{\zeta}$ is

$$
\leq|C \backslash B| \cdot k+|B \backslash A| \cdot l^{*} \leq|C| \cdot l^{*}=|C| \cdot n^{\xi} \cdot \mathbf{m}_{1.12}
$$

FACT 1.13. Suppose $A \leq B \leq C$.

(1) If $A \leq_{i} C$ then $B \leq_{i} C$.

(2) If $A \leq_{s} C$ then $A \leq_{s} B$.

(3) If $A<_{p r} C$ and $A \leq_{s} B \leq_{s} C$ then either $B=A$ or $B=C$.

Proof. Reread the definitions.

FACT 1.14. (1) If $A \leq_{s} B$ then there is some $n<\omega$ and a sequence $\left\langle A_{l}: l \leq n\right\rangle$ such that $A=A_{0}<_{p r} A_{1}<_{p r} \ldots<_{p r} A_{n}=B($ possibly $n=0)$.

(2) If $A<_{p r} C$ and $A<B<C$ then $B<{ }_{i} C$.

Proof. To prove (2), choose a maximal $B^{\prime}$ such that $B \leq_{i} B^{\prime} \leq C$; it exists as $C$ is finite (being in $\mathcal{K}_{\infty}$ ), and $B \leq_{i} B$ (by 1.11(1)). It follows that if $B^{\prime}<B^{\prime \prime} \leq C$ then $\neg B^{\prime} \leq_{i} B^{\prime \prime}$ (by 1.11(2)). Hence $B^{\prime} \leq_{s} C$. But $A<_{p r} C$, hence by Definition 1.4(5) we have $A<_{s} C$ so by $1.13(2), A<_{s} B^{\prime}$; so by the definition of $<_{p r}$ we have $B^{\prime}=C$, so $B \leq_{i} B^{\prime}=C$ as required. Part (1) is clear by the definition of $\leq_{p r}$ as $B$ is finite (being in $\mathcal{K}_{\infty}$ ). $\boldsymbol{v}_{1.14}$ 
ClaIm 1.15. $\mathcal{K}$ is weakly nice iff whenever $A<_{p r} B$ there is $\varepsilon \in \mathbb{R}^{+}$such that

$$
1=\lim _{n}\left[\operatorname{Prob}\left(\begin{array}{c}
\text { if } f_{0}: A \hookrightarrow \mathcal{M}_{n} \text { then there is } \mathcal{F} \text { with }|\mathcal{F}| \geq n^{\varepsilon} \text { and } \\
f_{1} \in \mathcal{F} \Rightarrow f_{0} \subseteq f_{1}: B \hookrightarrow \mathcal{M}_{n}
\end{array}\right)\right] .
$$

Proof. $\Rightarrow$ is obvious (as $A<_{p r} B$ implies $A<_{s} B$ ).

Let us prove $\Leftarrow$ : we have $A \leq_{s} B$ and by Fact 1.14(1) there is a sequence $A=A_{0}<_{p r} A_{1}<_{p r} \ldots<_{p r} A_{k}=B$. The proof is by induction on $k$. The induction step for $k>1$ is by the second proof of 1.12 and for $k=0$ it is 1.11(3). So assume $k=1$, hence $A<_{p r} B$. By Fact 1.14(2) if $A<B^{\prime} \leq B$ then $B^{\prime} \leq_{i} B$. Fix $p \in(0,1)_{\mathbb{R}}$. If $n$ is large enough then the probability of having both

(a) for every $f_{0}: A \hookrightarrow \mathcal{M}_{n}$ there are at least $n^{\varepsilon}$ different extensions $f_{1}^{i}$ satisfying $f_{0} \subseteq f_{1}^{i}: B \hookrightarrow \mathcal{M}_{n}$ and

(b) for every $a \in B \backslash A$ and $f_{0}^{+}: A \cup\{a\} \hookrightarrow \mathcal{M}_{n}$ there are at most $n^{\varepsilon / 2}$ different extensions $f_{2}^{i}$ satisfying $f_{0}^{+} \subseteq f_{2}^{i}: B \hookrightarrow \mathcal{M}_{n}$

is $\geq 1-p$ (for clause (b) use $A \cup\{a\}<_{i} B$ for every $a \in B \backslash A$, which holds by $1.14(2))$. Let $f_{0}: A \hookrightarrow \mathcal{M}_{n}$, and let $\left\langle f_{1}^{j}: j<j^{*}\right\rangle$ be a maximal family of pairwise disjoint extensions of $f_{0}$ to an embedding of $B$ into $\mathcal{M}_{n}$. Let $\mathcal{F}=\left\{f: f\right.$ is an embedding of $B$ into $\mathcal{M}_{n}$ extending $\left.f_{0}\right\}$. By (b) we have

$$
n^{\varepsilon} \leq|\mathcal{F}| \leq j^{*} \cdot|B \backslash A| \cdot|B \backslash A| \cdot n^{\varepsilon / 2} \text {. }
$$

Hence if $n$ is large enough, then $j^{*}>n^{\varepsilon / 3}$ (with probability $\geq 1-p$ ), and this is enough. 1.15

Claim 1.16. $\mathrm{cl}^{k, m}(A, M) \subseteq \mathrm{cl}^{k^{*}}(A, M)$ where $k^{*}=k^{m}$.

Proof. Define $k(l)$ by induction on $l \leq m: k(0)=1, k(1)=k$ and for $l<m$ (but $l \geq 1), k(l+1):=k(l) k$. For $l \leq m$ define $A_{l}=\mathrm{cl}^{k, l}(A, M)$. Now if $x \in A_{m}$ then there is some $l<m$ such that $x \in A_{l+1} \backslash A_{l}$. Let us prove by induction on $l \leq m$ that $x \in A_{l} \Rightarrow x \in \mathrm{cl}^{k(l)}(A, M)$. For $l=0$ and $l=1$ this is clear. If $x \in A_{l+1} \backslash A_{l}$ then there is $C$ with $|C| \leq k$ such that $x \in C$ and $C \cap A_{l}<_{i} C$. By the induction hypothesis, for $y \in C \cap A_{l}$ we have $y \in \mathrm{cl}^{k(l)}(A, M)$, hence there is $C_{y}$ with $\left|C_{y}\right| \leq k(l)$ such that $y \in C_{y}$ and $C_{y} \cap A \leq \leq_{i} C_{y}$. Let $C^{0}=\bigcup_{y \in C \cap A_{l}} C_{y} \cap A, C^{1}=\bigcup_{y \in C \cap A_{l}} C_{y}$ and $C^{2}=C^{1} \cup C$. As $|C| \leq k$, we get

$$
\left|C^{2}\right| \leq k(l) \cdot\left|C \cap A_{l}\right|+\left|C \backslash A_{l}\right| \leq k(l) \cdot k \leq k(l+1)
$$

so (as $x \in C^{2}$ ) it suffices to show that $C^{0} \leq_{i} C^{2}$ and by transitivity (i.e. by $1.11(2))$ it suffices to show that $C^{0} \leq_{i} C^{1}$ and that $C^{1} \leq_{i} C^{2}$. Why $C^{1} \leq_{i} C^{2}$ ? Because $C \cap A_{l} \leq_{i} C$ and $C \cap A_{l} \subseteq C^{1} \subseteq A_{l}$ and hence $C^{1} \leq_{i}$ $C^{1} \cup C=C^{2}$ by 1.11(4). Why $C^{0} \leq_{i} C^{1}$ ? Let $C \cap \overline{A_{l}}=\left\{y_{s}: s<r\right\}$. Now $C^{0} \leq_{i} C^{0} \cup C_{y_{0}}$ by $1.11(4)$ because $A \cap C_{y_{0}} \leq_{i} C_{y_{0}}$ and $A \cap C_{y_{0}} \subseteq C^{0}$ and 
similarly by induction

$$
C^{0} \leq_{i} C^{0} \cup C_{y_{0}} \leq_{i} C^{0} \cup C_{y_{0}} \cup C_{y_{1}} \leq_{i} \ldots \leq_{i} C^{0} \cup \bigcup_{s<r} C_{y_{s}}=C^{1} .
$$

So as $\leq_{i}$ is transitive $(1.11(2))$ we are done.

Claim 1.17. For every $\varepsilon \in \mathbb{R}^{+}$and $l, k, m$ we have

$$
1=\lim _{n}\left[\operatorname{Prob}\left(\begin{array}{l}
\text { if } A \in \mathcal{K}_{\infty},|A| \leq l \text { and } f: A \hookrightarrow \mathcal{M}_{n} \\
\text { then }\left|\mathrm{cl}^{k, m}\left(f(A), \mathcal{M}_{n}\right)\right|<n^{\varepsilon}
\end{array}\right)\right] .
$$

Proof. By the previous claim 1.16, we can assume $m=1$. Then this holds by Definitions 1.4(3) and 1.6.

FACT 1.18. (1) For every $A$ and $m, k$, for any $M \in \mathcal{K}$ if $f: A \hookrightarrow M$ then

$(\alpha) \mathrm{cl}^{k, m}(f(A), M) \leq_{1, k}^{i} \mathrm{cl}^{k, m+1}(f(A), M)$,

$(\beta)$ for some $m^{\prime}=m^{\prime}(k, m)$ we have

$$
f(A) \leq_{k, m^{\prime}}^{i} \mathrm{cl}^{k, m}(f(A), M)
$$

(we can get more),

$(\gamma) f(A) \leq_{i} \mathrm{cl}^{k, m}\left(f(A), M_{n}\right)$ or the second is not in $\mathcal{K}_{\infty}$.

(2) For every $m, k, l$ for some $r$ we have: for any $A \in \mathcal{K}_{\infty}$,

$$
1=\lim _{n}\left[\operatorname{Prob}\left(\text { if } f: A \hookrightarrow \mathcal{M}_{n} \text { then } f(A) \leq_{l, r}^{i} \mathrm{cl}^{k, m}\left(f(A), \mathcal{M}_{n}\right)\right)\right] \text {. }
$$

REMARK 1.19. In our main case $\mathcal{K}=\mathcal{K}_{\infty}$.

Recall for $1.18(1)(\gamma)$ that $\mathrm{cl}^{k, m}\left(f(A), \mathcal{M}_{n}\right)$ is in general not necessarily in $\mathcal{K}_{\infty}$.

Proof. (1) We leave the proof of $(\alpha)$ and $(\beta)$ to the reader. To prove clause $(\gamma)$, let $A_{0}=f(A)$ and for $l \leq m$ let $A_{l}=\mathrm{cl}^{k, l}(f(A), M)$, and assume $A_{l} \in \mathcal{K}_{\infty}$. So for $l<m$ we have $A_{l+1}=A_{l} \cup \bigcup_{j<m_{l}} C_{l, j}$ with $\left|C_{l, j}\right| \leq k$ and $A_{l+1} \cap C_{l, j} \leq_{i} C_{l, j}$. It follows by 1.11(4) that $\left\langle A_{l} \cup \bigcup_{i<j} C_{l, i}: j \leq m_{l}\right\rangle$ is $\leq_{i}$-increasing and $A_{l} \leq_{i} A_{l+1}$. By induction we get $A_{0} \leq_{i} A_{m}$, which is the desired conclusion.

(2) Read the proofs of $1.18(1)+1.16$. 1.18

REMARK 1.20. In a more general context the previous conclusion is part of the definition of " $\mathcal{K}$ is nice" and also $U$ of 1.23 below is a basic property (on the latter see [Sh 550]).

FACT 1.21. $\mathcal{K}_{\infty}$ is closed under isomorphisms and taking submodels.

FACT 1.22. For every $l, k, m$ there is a first order formula $\varphi\left(y, x_{0}, \ldots\right.$, $\left.x_{l-1}\right)$ such that for every $M \in \mathcal{K}$ and $b, a_{0}, \ldots, a_{l-1}$ in $M$ for any $f: A \hookrightarrow \mathcal{M}$

$$
M \models \varphi\left(b, a_{0}, \ldots, a_{l-1}\right) \quad \text { iff } \quad b \in \mathrm{cl}^{k, m}\left(\left\{a_{0}, \ldots, a_{l-1}\right\}, M\right) .
$$


Proof. By finiteness of $\tau$ (as $\tau_{\mathcal{K}}$ has no function symbols); or see proof of clause $(\beta)$ of 2.6 .

Definition 1.23. $C_{1} \bigcup_{B}^{D} C_{2}$ means: they are all submodels of $D \in \mathcal{K}$, and $C_{1} \cap C_{2} \subseteq B$ and for every relation symbol $R$ in $\tau$, if $\bar{a} \subseteq C_{1} \cup B \cup C_{2}$ and $R(\bar{a})$ holds then $\bar{a} \subseteq C_{1} \cup B$ or $\bar{a} \subseteq C_{2} \cup B$ (possibly both).

When $D$ is clear from the context we may omit it.

2. Abstract closure context. Here we are inside the $0-1$ context but without the $\leq_{i}$ and $\leq_{s}$ as defined in $\S 1$, however $\mathrm{cl}^{k}$ is given. The main result is a sufficient condition for having the 0-1 law or at least convergence. We have here some amount of freedom, so we give two variants of the main result of this section: $2.16,2.17$; we shall use 2.17 . Thus on a first reading one may skip Definitions 2.8 ("possible"), 2.9 and 2.10, Remark 2.11 and Lemma 2.16 in favour of the alternative development in Definitions 2.12, 2.13 and Lemma 2.17. Lemma 2.15 is needed in both cases and we have made the two independent at the price of some repetition. We want to "eliminate quantifiers" in a restricted sense: in the simple form we quantify only on the closure so each $\varphi(\bar{x})$ is equivalent to some $\psi_{\varphi}$ in which quantifiers are over $\mathrm{cl}^{k, m}(\bar{x})$; all this is for a random enough model where $\mathrm{cl}^{k, m}$ is "small", still it is not necessarily "tiny". The closure does not need to be in $\mathcal{K}_{\infty}$ (though in our application it is). The quantifier elimination result generalizes the result of [BlSh 528]. The chief additional ingredient in the proof here is the use of the addition (= Feferman-Vaught) theorem to analyze a pair of models in stable amalgamation; this is necessary as we do not have an a priori bound on the size of the closure, whereas there we have. Moreover, the argument in [BlSh 528] is simpler because $<_{i}$ is defined concretely from a dimension function and moreover it deals with the "nice" rather than almost nice case.

Note that the "simply" version $(2.20-2.24)$ is used in $\S 7$ (part II).

Note that in this section we could have forgotten about the probability distribution: just deal with elimination of quantifiers. Note that the assumption "cl is f.o. definable" $(2.2(\mathrm{~d}))$ is not serious: if it fails all we have to do is to allow " $y \in \mathrm{cl}^{k}(\bar{x})$ " as atomic formulas in $\psi_{\varphi}$.

Context 2.1. In this context in addition to $\mathfrak{K}$ (defined in 1.1) we have an additional basic operation cl which is a closure operation for $\mathcal{K}$ (see 2.2), so $\mathrm{cl}$ is in general not defined by Definition 1.6 and $\leq_{i}, \leq_{s}, \leq_{a}$ are defined by Definition 2.5 and in general are not the ones defined in Definition 1.4. However, we use $\mathcal{K}_{\infty}$ (from 1.4(1)). Lastly $\bigcup$ is as in 1.23 (can be axiomatized too and moreover generalized to the case of nonuniqueness, as in [Sh 550]). For simplicity assume $\tau_{\mathcal{K}}$ (the vocabulary of $\mathcal{K}$ ) is finite with no function symbols. In later sections $(\S 4-\S 7$ but not $\S 3)$ saying $\mathfrak{K}$ means cl is from $\S 1$. 
Definition 2.2. (1) We say cl is a closure operation for $\mathcal{K}$ if for $M \in \mathcal{K}$ and $k \in \mathbb{N}$ the operation $\mathrm{cl}^{k}(X, M)$ is defined if and only if $X \subseteq M$, and the operation satisfies:

(a) $X \subseteq \operatorname{cl}^{k}(X, M) \subseteq M$, and $X \subseteq Y \subseteq M \Rightarrow \mathrm{cl}^{k}(X, M) \subseteq$ $\mathrm{cl}^{k}(Y, M) \subseteq M$

(b) (i) if $\mathrm{cl}^{k}(X, M) \subseteq N \subseteq M$ then $\mathrm{cl}^{k}(X, N)=\mathrm{cl}^{k}(X, M)$,

(ii) if $X \subseteq N \subseteq M$ then $\operatorname{cl}^{k}(X, N) \subseteq \mathrm{cl}^{k}(X, M)$,

(c) for $k \leq l, \mathrm{cl}^{k}(X, M) \subseteq \mathrm{cl}^{l}(X, M)$,

(d) the relation " $b \in \mathrm{cl}^{k}(A, M)$ " is preserved by isomorphism.

(2) We say that the closure operation cl is f.o. definable if (e) below is true (and we assume this when not said otherwise):

(e) the assertion " $b \in \mathrm{cl}^{k}\left(\left\{a_{0}, \ldots, a_{l-1}\right\}, M\right)$ " is f.o. definable in $\mathcal{K}$, that is, there is a f.o. formula $\psi\left(y, x_{0}, \ldots, x_{l-1}\right)$ such that if $M \in$ $\mathcal{K}$ and $b, a_{0}, \ldots, a_{l-1} \in M$ then $b \in \operatorname{cl}^{k}\left(\left\{a_{0}, \ldots, a_{l-1}\right\}, M\right)$ iff $M \mid=\psi\left[y, x_{0}, \ldots, x_{l-1}\right]$.

(3) We say cl is transitive if for every $k$ for some $m$, for every $X \subseteq M \in \mathcal{K}$ we have $\operatorname{cl}^{k}\left(\mathrm{cl}^{k}(X, M), M\right) \subseteq \mathrm{cl}^{m}(X, M)$.

Definition 2.3. (1) For $X \subseteq M$ and $k, m \in \mathbb{N}$ we define $\mathrm{cl}^{k, m}(X, M)$ by induction on $m$ :

$$
\begin{aligned}
& \mathrm{cl}^{k, 0}(X, M)=X, \quad \mathrm{cl}^{k, 1}(X, M)=\mathrm{cl}^{k}(X, M), \\
& \mathrm{cl}^{k, m+1}(X, M)=\mathrm{cl}^{k, 1}\left(\mathrm{cl}^{k, m}(X, M), M\right)
\end{aligned}
$$

(if we write $\mathrm{cl}^{k, m-1}(X, M)$ and $m=0$ we mean $\mathrm{cl}^{k, 0}(X, M)=X$ ).

(2) We say the closure operation $\mathrm{cl}^{k}$ is $(l, r)$-local when: for $M \in \mathcal{K}$, $X \subseteq M$ and $Z \subseteq M$ if $Z \subseteq \mathrm{cl}^{k}(X, M),|Z| \leq l$ then for some $Y$ we have $Z \subseteq Y,|Y| \leq r$ and $\mathrm{cl}^{k}(Y \cap X, M \uparrow Y)=Y$.

(3) We say the closure operation cl is local if for every $k$, for some $r, \mathrm{cl}^{k}$ is $(1, r)$-local. We say that $\mathrm{cl}$ is simply local if $\mathrm{cl}^{k}$ is $(1, k)$-local for every $k$.

REMARK 2.4. (1) Concerning "possible in $\mathfrak{K}$ " (from Definition 2.8 below), in the main case $\mathcal{M}_{n, \bar{p}}^{0}$, it is degenerate, i.e. if $\bar{a} \subseteq N \in \mathcal{K}_{\infty}, B \subseteq N$ then $(N, B, \bar{a}, k, m)$ is possible. But for the case with the successor relation it has a real role.

(2) Note: if $\mathrm{cl}^{k}$ is $(1, r)$-local and " $y \in \mathrm{cl}^{k}\left(\left\{x_{1}, \ldots, x_{r}\right\}, M\right)$ " is f.o. definable then for every $m, s$ we have: " $y \in \mathrm{cl}^{k, m}\left(\left\{x_{1}, \ldots, x_{s}\right\}, M\right)$ " is f.o. definable.

(3) Clearly $\mathrm{cl}^{k, m_{1}}\left(\mathrm{cl}^{k, m_{2}}(X, M)\right)=\mathrm{cl}^{k, m_{1}+m_{2}}(X, M)$ and $k_{1} \leq k_{2} \wedge$ $m_{1} \leq m_{2} \Rightarrow \mathrm{cl}^{k_{1}, m_{1}}(A, M) \subseteq \mathrm{cl}^{k_{2}, m_{2}}(A, M)$.

(4) Note that if $\mathrm{cl}^{k}$ is $\left(l_{1}, r_{1}\right)$-local and $r_{2} \geq m r_{1}$ and $l_{2} \leq m l_{1}$ then $\mathrm{cl}^{k}$ is $\left(l_{2}, r_{2}\right)$-local. 
Definition 2.5 (For our 0-1 context $(\mathcal{K}, \mathrm{cl}$ ) with cl as a basic operation).

(1) $A \leq_{i} B$ if and only if $A \subseteq B \in \mathcal{K}_{\infty}$ and for some $k, m \in \mathbb{N}$ and every random enough $\mathcal{M}_{n}$ and for every embedding $g: B \hookrightarrow \mathcal{M}_{n}$ we have $g(B) \subseteq \mathrm{cl}^{k, m}\left(f(A), \mathcal{M}_{n}\right)$.

(2) $A<_{s} B$ if and only if $A \subseteq B \in \mathcal{K}_{\infty}$ and for every $k, m \in \mathbb{N}$ and random enough $\mathcal{M}_{n}$ and $f: A \hookrightarrow M_{n}$ there is $g$ such that $f \subseteq g$, and $g: B \hookrightarrow \mathcal{M}_{n}$ with $g(B) \cap \mathrm{cl}^{k, m}\left(f(A), \mathcal{M}_{n}\right)=f(A)$. We define $\leq_{p r}, \leq_{m}^{s}$, $\leq_{k, m}^{i}$ as in 1.4(5), (7), (8) respectively and $A<_{a} B$ means $A<B$ and $\neg\left(A<{ }_{s} B\right)$.

(3) $(\mathfrak{K}, \mathrm{cl})$ is weakly nice if for every $A \subseteq C \in \mathcal{K}_{\infty}$, for some $B$ we have $A \leq_{i} B \leq_{s} C$.

(4) We say $\mathfrak{K}$ (more exactly $(\mathfrak{K}, \mathrm{cl})$ ) is smooth $\left({ }^{1}\right)$ when:

$$
\begin{aligned}
& \text { if } A \subseteq B \subseteq N \in \mathcal{K}_{\infty}, A \subseteq C \subseteq N, B \bigcup_{A}^{N} C, \\
& \text { then } B<_{i} B \cup C \Leftrightarrow A<_{i} C
\end{aligned}
$$

(note that $\Leftarrow$ is always true).

(5) We say that $\mathrm{cl}^{k}$ is $r$-transparent if

$$
A \leq_{i} B \&|B| \leq r \Rightarrow \mathrm{cl}^{k}(A, B)=B .
$$

We say that $\mathrm{cl}$ is transparent if for every $r$ for some $k, \mathrm{cl}^{k}$ is $r$-transparent. We say that $\mathrm{cl}$ is simply transparent if for every $k, \mathrm{cl}^{k}$ is $k$-transparent.

FACT 2.6. Assume $\mathfrak{K}$ is a $0-1$ context (see 1.1) and $\mathrm{cl}$ is defined in 1.6. Then:

$(\alpha)$ cl is a closure operation for $\mathcal{K}_{\infty}$ (see Definitions 1.4(1) and 2.2(1)),

$(\beta) \mathrm{cl}$ is f.o. definable (for $\mathcal{K})$,

$(\gamma) \mathrm{cl}^{k, m}$ as defined in $1.6(\mathrm{c})$ and in 2.3 are equal,

$(\delta)$ cl is transitive,

$(\varepsilon)$ cl is simply local (see Definition 2.3(2),(3)),

$(\zeta) \mathrm{cl}$ is transparent, in fact $\mathrm{cl}^{k}$ is $k$-transparent for every $k$,

$(\eta) \leq_{i}$ as defined in 2.5(1) and in 1.4 are equal,

$(\theta)$ If in $\S 1, \mathfrak{K}$ is weakly nice (see Definition 1.9$)$ then $\left(\mathcal{K}_{\infty}, \mathrm{cl}\right)$ is weakly nice (by Definition 2.5(3)); if so then $\leq_{s}$ as defined in 2.5(2) and 1.4(4) are the same and $<_{a}$ as defined in 2.5(2) and in 1.4(6) are equal.

Proof. $(\alpha)$ We have to show that $\left(\mathcal{K}_{\infty}, \mathrm{cl}\right)$ from $\S 1$ satisfies clauses (a)-(d) from Definition 2.2(1).

(a) By the definition 1.6 of $\left(\mathcal{K}_{\infty}, \mathrm{cl}\right)$ the following holds: trivially $X \subseteq$ $\operatorname{cl}^{k}(X, M) \subseteq M$. Assume $X \subseteq Y \subseteq M$. If $b \in \operatorname{cl}^{k}(X, M)$ then for some $\bar{B}$ with $|B| \leq k$ and $b \in B, X \cap B \leq_{i} B$ by Definition 1.6. As $X \subseteq Y$ and

$\left({ }^{1}\right)$ Smoothness is not used in [Sh 550], but the closure there has an a priori bound, so the definitions there will be problematic here. See more in [Sh:F192]. 
$X \cap B \leq_{i} B$ we obtain $Y \cap B \leq_{i} B$ by Fact 1.13(1). So $B \subseteq \operatorname{cl}^{k}(Y, M)$ witnessing that $b \in \mathrm{cl}^{k}(Y, M)$. Hence $\operatorname{cl}^{k}(X, M) \subseteq \mathrm{cl}^{k}(Y, M)$.

(b) (i) First, let us show $\mathrm{cl}^{k}(X, N) \subseteq \mathrm{cl}^{k}(X, M)$. If $b \in \mathrm{cl}^{k}(X, N)$ then let $B$ witness it and $b \in B, B \subseteq N, B \cap X \leq_{i} B,|B| \leq k$. As $N \subseteq M$ the witness $B$ is in $M, B \cap X \leq_{i} B$ so $b \in \operatorname{cl}^{k}(X, M)$. Second we will show that $\mathrm{cl}^{k}(X, M) \subseteq \mathrm{cl}^{k}(X, N)$. If $b \in \mathrm{cl}^{k}(X, M)$ then there is $B$ witnessing it such that $b \in B \subseteq M, B \cap X \leq_{i} B,|B| \leq k$. Now clearly $B \subseteq \operatorname{cl}^{k}(X, M)$, hence by assumption $B \subseteq N$ so $b \in B \subseteq N, B \cap X \leq_{i} B,|B| \leq k$ and so $B$ witnesses $b \in \mathrm{cl}^{k}(X, N)$. So we get the result.

(ii) Included in the proof of clause (i).

(c) It follows immediately that $(\mathcal{K}, \mathrm{cl})$ holds by Definition 1.6.

(d) Easy.

$(\beta)$ We show that $(\mathcal{K}, \mathrm{cl})$ is f.o. definable. By Definition $2.2(\mathrm{~d})$ this means that for each $l$, there is a formula $\psi\left(y, x_{0}, \ldots, x_{l-1}\right)$ such that if $M \in \mathcal{K}$ and $b, a_{0}, \ldots, a_{l-1} \in M$ then: $b \in \operatorname{cl}^{k}\left(\left\{a_{0}, \ldots, a_{l-1}\right\}, M\right)$ iff $M \models$ $\psi\left(b, a_{0}, \ldots, a_{l-1}\right)$. It suffices to restrict ourselves to the case of $\left\langle a_{0}, \ldots, a_{l-1}\right\rangle$ with no repetition.

Let $\mathfrak{B}=\left\{(B, \bar{b}): B \in \mathcal{K}_{\infty}\right.$ has $\leq k$ elements, $\bar{b}$ is a sequence of length $\leq k$ listing the elements of $B$ without repetitions $\}$. On $\mathfrak{B}$ the relation $\cong$ (isomorphism) is defined. We say $\left(B^{\prime}, \bar{b}^{\prime}\right) \cong\left(B^{\prime \prime}, \bar{b}^{\prime \prime}\right)$ if there is an isomorphism $h$ from $B^{\prime}$ onto $B^{\prime \prime}$ mapping $\bar{b}^{\prime}$ onto $\bar{b}^{\prime \prime}$. Now $\cong$ is an equivalence relation on $\mathfrak{B}$ and $\mathfrak{B} / \cong$ is finite. So let $\left\{\left(B_{i}, \bar{b}_{i}\right): i<i^{*}\right\}$ be a set of representatives. Now $i^{*}$ is finite as $\tau$ is finite (actually locally finite suffices). When $k_{i}+1=\left|B_{i}\right|=\lg \left(\bar{b}_{i}\right)$ let $\varphi_{i}\left(x_{0}, \ldots, x_{k_{i}}\right)=$

$\bigwedge\left\{\theta\left(x_{0}, \ldots, x_{k_{i}}\right): \theta\right.$ is a basic formula (possibly with dummy variables) and $\left.B_{i}=\theta\left[b_{0}, \ldots, b_{k_{i}}\right]\right\}$.

Lastly

$$
\begin{aligned}
& \psi\left(y, x_{0}, \ldots, x_{l-1}\right)=\bigvee_{m<l} y=x_{m} \vee \\
& \bigvee_{i<i^{*} r<k_{i}}\left\{\left(\exists z_{0}, \ldots, z_{k_{i}}\right)\left(\bigwedge_{t<r} \bigvee_{m<l} x_{m}=z_{t} \wedge y=z_{k_{i}} \wedge \varphi_{i}\left(z_{0}, \ldots, z_{k_{i}}\right)\right):\right.
\end{aligned}
$$

$B_{i}$ has exactly $k_{i}+1$ members and $\left.B_{i} \uparrow\left\{b_{t}^{i}: t<r\right\} \leq_{i} B_{i}\right\}$.

$(\gamma)$ Trivial.

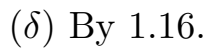

$(\varepsilon)$ Now, we will show that $\left(\mathcal{K}_{\infty}, \mathrm{cl}\right)$ is simply local. For this we have to show that $\mathrm{cl}^{k}$ is $(1, k)$-local for every $k$. Let $X \subseteq M \in \mathcal{K}$ be given and $Z \subseteq \mathrm{cl}^{k}(X, M)$ be such that $|Z| \leq 1$. If $Z=\emptyset$ let $Y=\emptyset$. So assume $Z=\{y\}$. As $y \in Z \subseteq \mathrm{cl}^{k}(X, M)$ there is a witness set $Y$ for $y \in \mathrm{cl}^{k}(X, M)$ 
so $Y \cap X \leq_{i} Y,|Y| \leq k$. As $Y \cap X \leq_{i} Y$, clearly $\operatorname{cl}(X \cap Y, Y)=Y$ and $Z=\{y\} \subseteq Y$ and $|Y| \leq k$ so we are done.

$(\zeta)$ Trivial by the definition of cl (Definition 1.6) and of transparency (Definition 2.5(5)).

$(\eta)$ First assume $A \leq_{i} B$ according to Definition 2.5 and we shall prove that $A \leq_{i} B$ according to Definition 1.4. So for some $k, m$ we have:

(*) for every random enough $\mathcal{M}_{n}$ and embedding $g: B \hookrightarrow \mathcal{M}_{n}$ we have $g(B) \subseteq \mathrm{cl}^{k, m}\left(g(A), \mathcal{M}_{n}\right)$.

Let $\varepsilon>0$. Let $\mathcal{M}_{n}$ be random enough and $f: A \hookrightarrow \mathcal{M}_{n}$. By $(*)$ and 1.16 if $g$ is an embedding of $B$ into $\mathcal{M}_{n}$ extending $f$ then we have $g(B) \subseteq$ $\mathrm{cl}^{k^{m}}\left(g(A), \mathcal{M}_{n}\right)$, hence

$$
\left|\operatorname{ex}\left(f, B, \mathcal{M}_{n}\right)\right| \leq\left|\mathrm{cl}^{k^{m}}\left(g(A), \mathcal{M}_{n}\right)\right|^{|B \backslash A|} .
$$

Let $\zeta=\varepsilon /(|B \backslash A|+1)$; now if $\mathcal{M}_{n}$ is random enough, then by 1.17 for every $g: B \hookrightarrow \mathcal{M}_{n}$ we have $\left|\mathrm{cl}^{k^{m}}\left(g(A), \mathcal{M}_{n}\right)\right|^{|B \backslash A|} \leq n^{\zeta}$, hence $\left|\operatorname{ex}\left(f, B, \mathcal{M}_{n}\right)\right| \leq$ $\left|n^{\zeta}\right| B \backslash A \mid \leq n^{\varepsilon}$. As $\varepsilon>0$ was arbitrary, we have proved that $A \leq_{i} B$ according to Definition 1.4.

Next assume $A \leq_{i} B$ according to Definition 1.4 and we shall prove that $A \leq_{i} B$ in the sense of Definition 2.5. Choose $k=|B|$ and $m=1$, so $\mathrm{cl}^{k, m}=\mathrm{cl}^{k}$. So let $\mathcal{M}_{n}$ be random enough, and $g: B \hookrightarrow \mathcal{M}_{n}$. Recall that $\mathrm{cl}^{k}\left(g(A), \mathcal{M}_{n}\right)=\bigcup\left\{C: C \subseteq \mathcal{M}_{n},|C| \leq k\right.$ and $\left.C \cap A \leq{ }_{i} C\right\}$, so $g(B)$ can serve as such $C$, hence $g(B) \subseteq \operatorname{cl}^{k}\left(g(A), \mathcal{M}_{n}\right)$.

$(\theta)$ We shall use clause $(\eta)$ freely. First assume that $\mathcal{K}$ is weakly nice according to Definition 1.9 and we shall prove that $(\mathcal{K}, \mathrm{cl})$ is weakly nice in the sense of Definition 2.5(3). So assume $A \leq B$. We can find $C$ such that $A \leq_{i} C \leq B$ and for no $C^{\prime}, A \leq_{i} C^{\prime} \leq B, C \subset C^{\prime}$; it exists as $A \leq_{i} A \leq B$ and $B$ is finite. By 1.11(2) for no $C^{\prime}$, do we have $C<_{i} C^{\prime} \leq B$, hence $C \leq_{s} B$ in the sense of Definition 1.4, so it is enough to prove that $C \leq_{s} B$ in the sense of Definition 2.5(2), and we can assume $C \neq B$ so $C<_{s} B$. Let $k, m$ be given. As we are assuming that $\mathcal{K}$ is weakly nice according to Definition 1.9 and $C<_{s} B$ in the sense of Definition 1.4(4) we find that there is an $\varepsilon \in \mathbb{R}^{+}$such that

$1=\lim _{n}\left[\operatorname{Prob}\left(\begin{array}{l}\text { if } f_{0}: C \hookrightarrow \mathcal{M}_{n} \text { then there is } \mathcal{F} \text { with }|\mathcal{F}| \geq n^{\varepsilon} \text { and } \\ \text { (i) } f \in \mathcal{F} \Rightarrow f_{0} \subseteq f: B \hookrightarrow \mathcal{M}_{n}, \\ \text { (ii) } f^{\prime} \neq f^{\prime \prime} \in \mathcal{F} \Rightarrow \operatorname{Rang}\left(f^{\prime}\right) \cap \operatorname{Rang}\left(f^{\prime \prime}\right)=\operatorname{Rang}\left(f_{0}\right)\end{array}\right)\right]$.

As $\mathcal{M}_{n}$ is random enough and $f: C \hookrightarrow \mathcal{M}_{n}$, there is $\mathcal{F}$ as above for $B$ with $|\mathcal{F}| \geq n^{\varepsilon}$; but by 1.16 also

$$
\left|\mathrm{cl}^{k, m}\left(f(C), \mathcal{M}_{n}\right)\right| \leq\left|\mathrm{cl}^{l}\left(f(C), \mathcal{M}_{n}\right)\right|
$$


for $l=k^{m}$ and by 1.7 we have

$$
\left|\mathrm{cl}^{k^{m}}\left(f(C), \mathcal{M}_{n}\right)\right|<n^{\varepsilon}
$$

so $\left|\mathrm{cl}^{k, m}\left(f(C), \mathcal{M}_{n}\right)\right|<n^{\varepsilon}$.

As the sequence $\langle\operatorname{Rang}(g) \backslash \operatorname{Rang}(f): g \in \mathcal{F}\rangle$ lists a family of $\geq n^{\varepsilon}>$ $\left|c l^{k, m}\left(f(C), \mathcal{M}_{n}\right)\right|$ pairwise disjoint subsets of $\mathcal{M}_{n}$, for some $g \in \mathcal{F}$, we have: $\operatorname{Rang}(g) \cap \operatorname{Rang}(f)$ is disjoint from $\mathrm{cl}^{k, m}\left(f(C), \mathcal{M}_{n}\right)$. So $g$ is as required in Definition 2.5(2); so we have proved $C \leq_{s} B$ according to Definition 2.5 , hence we have finished proving $\left(\mathcal{K}_{\infty}, \mathrm{cl}\right)$ is weakly nice according to Definition 2.5(3).

So we have proved the implication between the two versions of weakly nice. Second, assuming $\mathfrak{K}$ is weakly nice according to Definition 1.9 , we still have to say why the two versions of $\leq_{s}$ (by Definition 1.4(4) and 2.5(2)) are equivalent. Now if $C \leq_{s} B$ in the sense of Definition 1.4(4) then $C \leq_{s} B$ according to Definition 2.5(2) has been proved within the proof above that $\mathcal{K}$ is weakly nice. Lastly assume $A \leq_{s} B$ according to Definition 2.5(2); now if $A<_{i} C \leq B$ we get a contradiction directly from Definition 2.5(2): but this confirms $A \leq_{s} B$ according to Definition 1.4(4).

Lastly we leave the statement on $<_{a}$ to the reader. $\mathbf{m}_{2.6}$

REMARK 2.7. (1) Note that the assumption " $\mathfrak{K}$ is weakly nice" is very natural in the applications we have in mind.

(2) Why have we not proved the equivalence of the two versions of weakly nice in 2.6( $\theta)$ ? We can define the following 0-1 context $\mathfrak{K}$ : let $\mathcal{M}_{n}$ be $\mathcal{M}_{n, \bar{p}}^{0}$ if $n$ is even with $p_{n}=1 / n^{\alpha}, \alpha \in(0,1)_{\mathbb{R}}$ irrational (except $p_{1}=1 / 2^{\alpha}$ ), and let $\mathcal{M}_{n}$ be the random graph with probability $1 / 2$ if $n$ is odd. Now in $\S 1$, $\mathcal{K}_{\infty}$ is the family of finite graphs, and $A \leq_{i} B$ iff $A=B$ (using the odd $n$ 's). Hence $\mathrm{cl}^{k}(A, M)=A$ so clearly $A<B \Rightarrow A<_{s} B$ according to 1.4 , hence weak niceness in the sense of $2.5(3)$ holds trivially but weak niceness in the sense of Definition 1.9 fails.

(3) Note that in Definitions 2.8, 2.9, 2.12 below the "universal" demand says about a given situation in random enough $\mathcal{M}_{n}$ whereas the "existential demand" implicit in goodness deals with extensions of an embedding into $\mathcal{M}_{n}$.

(4) We would like to show that for every formula $\varphi(\bar{x})$ (f.o. in the vocabulary $\tau_{\mathcal{K}}$ ) there are (f.o.) $\psi_{\varphi}(\bar{x})$ and $k=k_{\varphi}, m=m_{\varphi}$ such that

$(*)_{\varphi}$ for every random enough $\mathcal{M}_{n}$ and $\bar{a} \in \lg (\bar{x}) \mathcal{M}_{n}$ we have $\mathcal{M}_{n} \mid=\varphi[\bar{a}]$ $\Leftrightarrow \mathcal{M}_{n}\left\lceil\mathrm{cl}^{k, m}\left(\bar{a}, \mathcal{M}_{n}\right) \models \psi_{\varphi}(\bar{x})\right.$.

Naturally enough we shall do it by induction on the quantifier depth of $\varphi$ and the nontrivial case is $\varphi(\bar{x})=(\exists y) \varphi_{1}(\bar{x}, y)$, and we assume $\psi_{\varphi_{1}}(\bar{x}, y)$, $k_{\varphi_{1}}, m_{\varphi_{1}}$ are well defined. So we should analyze the situation: $\mathcal{M}_{n}$ is random enough, $\bar{a} \in \lg (\bar{x})\left(\mathcal{M}_{n}\right), \mathcal{M}_{n} \models \varphi[\bar{a}]$ so there is $b \in \mathcal{M}_{n}$ such that 
$\mathcal{M}_{n}=\varphi_{1}[\bar{a}, b]$, and we split it into two cases according to the satisfaction of a suitable statement on a suitable neighbourhood of $\bar{a}$, i.e., $\mathrm{cl}^{k^{\prime}, m^{\prime}}\left(\bar{a}, \mathcal{M}_{n}\right)$. If $b$ belongs to a small enough neighbourhood of $\bar{a}$ this should be clear. If not we would like to find a suitable situation (really a set of possible situations, with a bound on their number depending just on $\varphi$ ) to guarantee the existence of an element $b$ with $\mathrm{cl}^{k_{\varphi_{1}}, m_{\varphi_{1}}\left(\bar{a} b, \mathcal{M}_{n}\right)}$ satisfying $\psi_{\varphi_{1}}(\bar{a}, b)$. Now in general the $\mathrm{cl}^{k_{\varphi_{1}}, m_{\varphi_{1}}}$ can be of large cardinality (for $\varphi$, i.e. depending on $\left.\mathcal{M}_{n}\right)$. In the nice case we are analyzing, to find such a witness $b$ outside a small neighbourhood of $\bar{a}$ it will suffice to look at $\mathrm{cl}^{k_{\varphi_{1}}, m_{\varphi_{1}}}\left(\bar{a} b, \mathcal{M}_{n}\right)$ essentially with small cardinality. Why only essentially? Because maybe $\mathrm{cl}^{k_{\varphi}, m_{\varphi}}\left(\bar{a}, \mathcal{M}_{n}\right)$ is already large, so what we should have is something like: $\mathrm{cl}^{k_{\varphi_{1}}, m_{\varphi_{1}}}\left(\bar{a} b, \mathcal{M}_{n}\right) \backslash \mathrm{cl}^{k_{\varphi}, m_{\varphi}}\left(\bar{a}, \mathcal{M}_{n}\right)$ can be replaced by a set of small cardinality. For this we need $U$ (the relation of free amalgamation) to hold, possibly replacing $\mathrm{cl}^{k_{\varphi}, m_{\varphi}}\left(\bar{a}, \mathcal{M}_{n}\right)$ by a subset (in $\S 3$ we can make it arbitrary, here quite definable) and the amalgamation base has an a priori bound. By the addition theorem $(2.15)$ we may replace $\left(B^{*}, b\right)_{b \in B}$ by similar enough $\left(B^{\prime}, b\right)_{b \in B}$ (in particular when $B^{*} \in \mathcal{K}_{\infty}$ ) so we need to express in such a situation something like: $B^{*}$ exists over $B$ (we can say such a $B$ exists by clause (b) of 2.8(4) using quantifiers on $\left.\mathrm{cl}^{k, m}\left(\bar{a}, \mathcal{M}_{n}\right)\right)$. Well, $B \leq_{s} B^{*}$ is a good approximation. But this does not say that $\operatorname{cl}\left(\bar{a} b, \mathcal{M}_{n}\right)$ is suitable. So we need to say first that the closure of $\bar{a} b$ in essentially $B^{*} \cup B_{2}$, where $B_{2}=\operatorname{cl}^{k_{\varphi_{1}}, m_{2}}\left(\bar{a}, \mathcal{M}_{n}\right)$, obeys a version of the addition theorem, and secondly that $B^{*}$ sits in $\mathcal{M}_{n}$ in such a way that the closure is right. All this is carried out in Definition 2.8(4) (of "good" saying: we have a tuple in a situation which exists whenever a copy of $B$ as above exists) and 2.9 (when there are $B$ etc. as above). The proof is carried out in 2.16 .

(5) Defining "good", by demanding the existence of the embedding $g$ : $B^{*} \hookrightarrow \mathcal{M}_{n}$ extending $f: B \hookrightarrow \mathcal{M}_{n}$, we demand on $f$ just that it is an embedding. We may impose requirements of the form $\mathrm{cl}^{k_{i}, m_{i}}\left(f\left(B_{i}\right), \mathcal{M}_{n}\right) \subseteq$ $f(B)$ or $\mathrm{cl}^{k_{i}, m_{i}}\left(f\left(B_{i}\right), \mathcal{M}_{n}\right) \cap f(B)=f\left(C_{i}\right)$ for some $B_{i}, C_{i} \subseteq B$. This makes it easier for a tuple to be good, thus giving a version of almost nice covering more cases. In another possible strengthening we do not replace $B^{*}$ by $B^{\prime} \in$ $\mathcal{K}_{\infty}$ of bounded cardinality but look at it as a family of possible ones all similar in the relevant sense. On the other hand we may like simpler versions which are pursued in 2.13, 2.17.

(6) Note that if $\mathrm{cl}^{k}$ is $r$-transparent and $A \subseteq M \in \mathcal{K}$ then $\mathrm{cl}^{k}(A, M) \supseteq$ $\bigcup\left\{C \subseteq M: C \cap A \leq_{i} C\right.$ and $\left.|C| \leq r\right\}$. [Why? if $C \subseteq M, C \cap A \leq_{i} C$ and $|C| \leq r$ then: first $\mathrm{cl}^{k}(C \cap A, C)=C$ as $\mathrm{cl}^{k}$ is $r$-transparent; second $\mathrm{cl}^{k}(C \cap$ $A, C) \subseteq \mathrm{cl}^{k}(C \cap A, M)$ by (b)(ii) of Definition 2.2(1), third $\mathrm{cl}^{k}(C \cap A, M) \subseteq$ $\mathrm{cl}^{k}(A, M)$ as $C \cap A \subseteq M$ by clause (a) of Definition 2.2(1); altogether we are done.] Note that if $\mathrm{cl}^{k}$ is $(1, r)$-local we can prove the other inclusion. So 
obviously if $(\mathfrak{K}, \mathrm{cl})$ is simply local and simply transparent (and $\tau_{\mathcal{K}}$ is finite or at least locally finite of course), then cl is f.o. definable.

Definition 2.8. (1) We say $(N, B, \bar{B}, k)$ is possible for $(\mathfrak{K}, \mathrm{cl})$ if:

(a) $\bar{B}=\left\langle B_{i}: i<\lg (\bar{B})\right\rangle, B_{i} \subseteq N \in \mathcal{K}_{\infty}, B \subseteq N$ and $\mathrm{cl}^{k}\left(B_{i}, N\right) \subseteq$ $B_{i+1}$ for $i<\lg (\bar{B})-1$,

(b) it is not true that: for every random enough $\mathcal{M}_{n}$, for no embed$\operatorname{ding} f: N \hookrightarrow \mathcal{M}_{n}$, do we have: for $i<\lg (\bar{B})-1$,

$$
\mathrm{cl}^{k}\left(f\left(B_{i}\right), \mathcal{M}_{n}\right) \subseteq f\left(\mathrm{cl}^{k}\left(B_{i}\right), N\right) \cup \mathrm{cl}^{k}\left(f(B), \mathcal{M}_{n}\right) .
$$

(2) If we write $(N, C, B, k)$ we mean $\left(N, C,\left\langle B, \operatorname{cl}^{k}(B, N)\right\rangle, k\right)$.

(3) We say $(N, B, \bar{a}, k, m)$ is possible for $\mathfrak{K}$ if $(N, B, \bar{B}, k)$ is possible for $\mathfrak{K}$ where $\bar{B}=\left\langle\mathrm{cl}^{k, i}(\bar{a}, N): i \leq m\right\rangle$.

(4) We say that the tuple $\left(B^{*}, B, B_{0}, B_{1}, k, m_{1}, m_{2}\right)$ is $\operatorname{good}$ for $(\mathfrak{K}, \mathrm{cl})$ if

(a) $B \leq B^{*} \in \mathcal{K}_{\infty}$ and $B_{0} \leq B_{1} \leq B^{*} \in \mathcal{K}_{\infty}$,

(b) for every random enough $\mathcal{M}_{n}$ we have: if $f: B \hookrightarrow \mathcal{M}_{n}$ then there is an extension $g$ of $f$ satisfying $g: B^{*} \hookrightarrow \mathcal{M}_{n}$ and

( $\alpha) g\left(B^{*}\right) \cap \mathrm{cl}^{k, m_{2}}\left(f(B), \mathcal{M}_{n}\right)=f(B)$,

$(\beta) \mathrm{cl}^{k, m_{1}}\left(g\left(B_{0}\right), \mathcal{M}_{n}\right) \subseteq g\left(B_{1}\right) \cup \mathrm{cl}^{k, m_{2}}\left(g(B), \mathcal{M}_{n}\right)$,

$(\gamma) \mathcal{M}_{n}\left\lceil g\left(B^{*}\right) \quad \mathcal{M}_{n} \quad \mathcal{M}_{n}\left\lceil\mathrm{cl}^{k, m_{2}}\left(f(B), \mathcal{M}_{n}\right)\right.\right.$, $\mathcal{M}_{n}\lceil f(B)$

$(\delta) \mathrm{cl}^{k, m}\left(g\left(B_{0}\right), g\left(B^{*}\right)\right)=g\left(B^{*}\right) \cap \mathrm{cl}^{k, m}\left(g\left(B_{0}\right), \mathcal{M}_{n}\right)$ for $m \leq m_{2}$; if we omit $B_{1}$ we mean $\mathrm{cl}^{k, m_{2}}\left(B_{0}, B^{*}\right)$.

Definition 2.9. The 0 -1 context $\mathfrak{K}$ with closure $\mathrm{cl}$ (or the pair $(\mathfrak{K}, \mathrm{cl}$ ) or $\mathfrak{K}$ when $\mathrm{cl}$ is understood) is almost nice if it is weakly nice and:

(A) the universal demand: for every $k, m_{0}$ and $l, l^{\prime}$ there are $m^{*}=$ $m^{*}\left(k, m_{0}, l, l^{\prime}\right)>m_{0}, k^{*}=k^{*}\left(k, m_{0}, l, l^{\prime}\right) \geq k$ and $t=t\left(k, m_{0}, l, l^{\prime}\right)$ such that, for every random enough $\mathcal{M}_{n}$ we have: if $\bar{a} \in{ }^{l}\left|\mathcal{M}_{n}\right|$ and $b \in \mathcal{M}_{n} \mid$ $\mathrm{cl}^{k^{*}, m^{*}}\left(\bar{a}, \mathcal{M}_{n}\right)$ then there are $m_{2} \in\left[m_{0}, m^{*}\right]$ and $m_{1} \leq m^{*}-m_{2}$ and $B \subseteq \operatorname{cl}^{k, m_{1}}\left(\bar{a}, \mathcal{M}_{n}\right)$ and $B^{*} \subseteq \mathcal{M}_{n}$ such that:

$(\alpha)|B| \leq t$ and $\bar{a} \subseteq B$,

( $\beta) B^{*}=\left[\mathrm{cl}^{k, m_{0}}\left(\bar{a} \bar{b}, \mathcal{M}_{n}\right) \backslash \mathrm{cl}^{k, m_{2}}\left(B, \mathcal{M}_{n}\right)\right] \cup B$ so necessarily $b \in B^{*}$ and $\bar{a} \subseteq B^{*}$ (see 2.11 below),

$(\gamma) B<_{s} B^{*}$ or at least:

for every first order formula $\varphi=\varphi\left(\ldots, x_{a}, \ldots\right)_{a \in B}$ of quantifier depth $\leq l^{\prime}$ there is $B^{\prime}$ such that $B<_{s} B^{\prime}\left(\right.$ so $\left.B^{\prime} \in \mathcal{K}_{\infty}\right)$ and

$$
\begin{aligned}
B^{*} & =\varphi(\ldots, a, \ldots)_{a \in B} \quad \text { iff } \quad B^{\prime} \models \varphi(\ldots, a, \ldots)_{a \in B}, \\
(\delta) \mathcal{M}_{n}\left\lceil B^{*}\right. & \bigcup_{\mathcal{M}_{n}\lceil B}^{\mathcal{M}_{n}} \mathcal{M}_{n}\left\lceil\mathrm{cl}^{k, m_{2}}\left(B, \mathcal{M}_{n}\right),\right.
\end{aligned}
$$




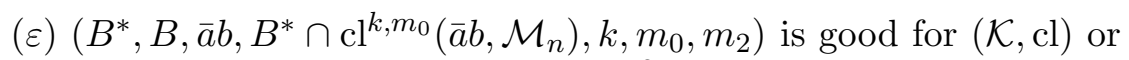
at least for some $B^{\prime}, B^{\prime \prime}$ we have $\left({ }^{2}\right)$ :

(i) $\left(B^{\prime}, B, \bar{a} b, B^{\prime \prime}, k, m_{1}, m_{2}\right)$ is good for $(\mathcal{K}, \mathrm{cl})$,

(ii) $\left.\left(B^{*}, \mathrm{cl}^{k, m_{0}}\left(\bar{a} b, \mathcal{M}_{n}\right) \cap B^{*}\right), b, c\right)_{c \in B} \equiv_{l^{\prime}}\left(B^{\prime}, B^{\prime \prime}, b, c\right)_{c \in B}$,

$(\zeta)$ for $m \leq m_{2}$ we have

$$
\mathrm{cl}^{k, m}\left(\bar{a} b, B^{*}\right)=B^{*} \cap \mathrm{cl}^{k_{\varphi_{1}}, m}\left(\bar{a} b, \mathcal{M}_{n}\right) .
$$

[So by clause $(\zeta)$, in clause $(\varepsilon)$ we can omit $B^{*} \cap \mathrm{cl}^{k, m_{0}}\left(\bar{a} b, \mathcal{M}_{n}\right)$ and $B^{\prime \prime}$, at the expense of increasing $l^{\prime}$.]

Definition 2.10. If in Definition 2.9 above, $k^{*}=k$ in clause $(\mathrm{A})$ then we say: $\mathfrak{K}$ is almost nice $k$-preserving.

REMARK 2.11. (1) Note that if $\mathcal{K}=\mathcal{K}_{\infty}$ and $\mathrm{cl}$ is local (or just $\mathrm{cl}^{k}$ is $\left(l_{k}, r_{k}\right)$-local for each $k$ ) (which holds in the cases we are interested in) then in clauses $(\gamma),(\varepsilon)$ of $(\mathrm{A})$ in Definition 2.9 above the two possibilities are close.

(2) Why in $2.9(\mathrm{~A})(\beta)$ do we have "necessarily $b \in B^{*}$ "? Because

$$
b \in \operatorname{Rang}(\bar{a} b) \subseteq \mathrm{cl}^{k, m_{0}}\left(\bar{a} b, \mathcal{M}_{n}\right)
$$

and

$$
\begin{aligned}
\operatorname{cl}^{k, m_{2}}\left(B, \mathcal{M}_{n}\right) & \subseteq \mathrm{cl}^{k, m_{2}}\left(\mathrm{cl}^{k, m_{1}}\left(\bar{a}, \mathcal{M}_{n}\right)\right) \subseteq \mathrm{cl}^{k, m_{1}+m_{2}}\left(\bar{a}, \mathcal{M}_{n}\right) \\
& \subseteq \mathrm{cl}^{k, m^{*}}\left(\bar{a}, \mathcal{M}_{n}\right) \subseteq \mathrm{cl}^{k^{*}, m^{*}}\left(\bar{a}, \mathcal{M}_{n}\right)
\end{aligned}
$$

and $b$ does not belong to the latter.

(3) Why do we use $\mathrm{cl}^{k, m_{2}}\left(B, \mathcal{M}_{n}\right)$ ? Part of our needs is that this set is definable from $B$ without $b$.

(4) In Definition $2.9(\mathrm{~A})(\gamma)$, there is one $B^{\prime}$ for all such $\varphi$ (why? because the set of f.o. formulas of quantifier depth $l$ is closed under Boolean combinations) so for some $B^{\prime} \in \mathcal{K}_{\infty}$ we have $B \leq_{s} B^{\prime}$, and $\left(B^{\prime}, c\right)_{c \in B} \equiv_{l}\left(B^{*}, c\right)_{c \in B}$. So we could have phrased clause (ii) of $(\mathrm{A})(\varepsilon)$ in the same way as clause $(\gamma)$.

In our main case, also the following variant of the property applies (see 2.18 below).

Definition 2.12. (1) We say that the quadruple $\left(N, B,\left\langle B_{0}, B_{1}\right\rangle, k\right)$ is simply good for $(\mathfrak{K}, \mathrm{cl})$ if $\left(B, B_{0}, B_{1} \leq N \in \mathcal{K}_{\infty}\right.$ and $)$ for every random enough $\mathcal{M}_{n}$, for every embedding $f: B \hookrightarrow \mathcal{M}_{n}$ there is an extension $g$ of $f$ satisfying $g: N \hookrightarrow \mathcal{M}_{n}$ such that:

$$
\begin{aligned}
& \text { (i) } g(N) \cap \mathrm{cl}^{k}\left(f(B), \mathcal{M}_{n}\right)=f(B), \\
& \text { (ii) } g(N) \underset{f(B)}{\cup} \operatorname{cl}^{k}\left(f(B), \mathcal{M}_{n}\right) \\
& \text { (iii) } \operatorname{cl}^{k}\left(g\left(B_{0}\right), \mathcal{M}_{n}\right) \subseteq g\left(B_{1}\right) \cup \mathrm{cl}^{k}\left(g(B), \mathcal{M}_{n}\right)
\end{aligned}
$$

$\left(^{2}\right) M_{1} \equiv_{l^{\prime}} M_{2}$ means: $M_{1}, M_{2}$ satisfy the same f.o. sentences of quantifier depth $\leq l^{\prime}$. 
(natural but not used is $\left.\mathrm{cl}^{k}\left(g\left(B_{0}\right), \mathcal{M}_{n}\right) \cap g(N)=g\left(\mathrm{cl}^{k}\left(B_{0}, N\right)\right)\right)$. If we write $B_{0}$ instead $\left\langle B_{0}, B_{1}\right\rangle$, we mean $B_{1}=N$.

(2) We say that $\left(N, B,\left\langle B_{0}, B_{1}\right\rangle, k, k^{\prime}\right)$ is simply good if part (1) holds with (iii) replaced by

$(\text { iii) })^{\prime} \mathrm{cl}^{k}\left(g\left(B_{0}\right), \mathcal{M}_{n}\right) \subseteq g\left(B_{1}\right) \cup \mathrm{cl}^{k^{\prime}}\left(g(B), \mathcal{M}_{n}\right)$.

Definition 2.13. (1) The 0-1 context with closure $(\mathfrak{K}, \mathrm{cl})$ is simply almost nice if it is weakly nice and:

(A) the universal demand: for every $k$ and $l, l^{\prime}$ there are $m^{*}=m^{*}\left(k, l, l^{\prime}\right)$, $k^{*}=k^{*}\left(k, l, l^{\prime}\right) \geq k$ and $t=t\left(k, l, l^{\prime}\right)$ such that for every random enough $\mathcal{M}_{n}$ we have: if $\bar{a} \in{ }^{l}\left|\mathcal{M}_{n}\right|$ and $b \in \mathcal{M}_{n} \backslash \mathrm{cl}^{k^{*}, m^{*}}\left(\bar{a}, \mathcal{M}_{n}\right)$ then there are $B \subseteq \mathrm{cl}^{k^{*}, m^{*}}\left(\bar{a}, \mathcal{M}_{n}\right)$ and $B^{*} \subseteq \mathcal{M}_{n}$ such that:

$(\alpha)|B| \leq t$ and $\bar{a} \subseteq B$ and $\operatorname{cl}^{k}\left(B, \mathcal{M}_{n}\right) \subseteq \operatorname{cl}^{k^{*}, m^{*}}\left(\bar{a}, \mathcal{M}_{n}\right)$,

( $\beta) B^{*}=\left[\operatorname{cl}^{k}\left(\bar{a} b, \overline{\mathcal{M}}_{n}\right) \backslash \operatorname{cl}^{k}\left(B, \mathcal{M}_{n}\right)\right] \cup B$ (or at least $B^{*} \supseteq\left[\mathrm{cl}^{k}\left(\bar{a} b, \mathcal{M}_{n}\right)\right.$ $\left.\left.\backslash \mathrm{cl}^{k}\left(B, \mathcal{M}_{n}\right)\right] \cup B\right)$,

( $\gamma) B<_{s} B^{*}\left(\right.$ so $\left.B^{*} \in \mathcal{K}_{\infty}\right)$ or at least for every first order formula $\varphi=\varphi\left(x_{b}, \ldots, x_{a}, \ldots\right)_{a \in B}$ of quantifier depth $\leq l^{\prime}$ there is $B^{\prime}$ such that $B<_{s} B^{\prime}\left(\right.$ so $\left.B^{\prime} \in \mathcal{K}_{\infty}\right)$ and

$$
B^{*} \models \varphi(b, \ldots, c, \ldots)_{c \in B} \quad \text { iff } \quad B^{\prime} \models \varphi(b, \ldots, c, \ldots)_{c \in B},
$$

or even, but actually equivalently,

$$
\left(B^{*}, b, \ldots, c, \ldots\right)_{c \in B} \equiv_{l^{\prime}}\left(B^{\prime}, b, \ldots, c, \ldots\right)_{c \in B}
$$

$(\delta) \mathcal{M}_{n}\left\lceil B^{*} \stackrel{\mathcal{M}_{n}}{\bigcup} \mathcal{M}_{n}\left\lceil\mathrm{cl}^{k}\left(B, \mathcal{M}_{n}\right)\right.\right.$

$$
\mathcal{M}_{n} \uparrow B
$$

( $\varepsilon) B^{*} \in \mathcal{K}_{\infty}$ and $\left(\mathcal{M}_{n}\left\lceil B^{*}, B, \bar{a} b, k\right)\right.$ is simply good for $(\mathfrak{K}, \mathrm{cl})$ or at least for some $B^{\prime}, b^{\prime}$ we have:

(i) $\left(B^{\prime}, B, \bar{a} b^{\prime}, k\right)$ is simply good for $(\mathfrak{K}, \mathrm{cl})$,

(ii) $\left(B^{*}, b, \ldots, c, \ldots\right)_{c \in B} \equiv_{l^{\prime}}\left(B^{\prime}, b^{\prime}, \ldots, c, \ldots\right)_{c \in B}$.

(2) If above always $k^{*}=k$ we say: $\mathfrak{K}$ is simply almost nice depth preserving.

(3) We say that $(\mathfrak{K}, \mathrm{cl})$ is simply nice (i.e. without "almost") if 2.13(1) holds but we omit clause $(\varepsilon)$ and add:

(B) If $B<_{s} B^{*}$ and $k \in \mathbb{N}$ then $\left(B^{*}, B, B^{*}, k\right)$ is simply good.

(C) $\mathcal{K}_{\infty}=\mathcal{K}$ (or at least if $A \in \mathcal{K}_{\infty}$ and $k, m \in \mathbb{N}$ then for any random enough $\mathcal{M}_{n}$ for any $f: A \hookrightarrow \mathcal{M}_{n}, \mathrm{cl}^{k, m}\left(A, \mathcal{M}_{n}\right) \in \mathcal{K}_{\infty}$.

Similarly in Definition 2.9 for "nice".

REMARK 2.14. (1) In 2.13(1) we can weaken the demands (and call $(\mathcal{K}, \mathrm{cl})$ simply $^{\otimes}$ almost nice $)$ : get also $k^{\otimes}=k^{\otimes}\left(k, l, l^{\prime}\right) \in \mathbb{N}$, replace in clause $(\beta) \mathrm{cl}^{k}\left(B, \mathcal{M}_{n}\right)$ by $\mathrm{cl}^{k^{\otimes}}\left(b, \mathcal{M}_{n}\right)$ and replace $(\varepsilon)$ by 
$\left(\varepsilon^{\prime}\right)\left(B^{\prime}, B, \bar{a} b, k, k^{\otimes}\right)$ is simply good for $(\mathfrak{K}, \mathrm{cl})($ see $2.12(2))$ or at least for some $B^{\prime}, b^{\prime}$ we have:

(i) $\left(B^{\prime}, B, \bar{a} b^{\prime}, k, k^{\otimes}\right)$ is simply ${ }^{\otimes} \operatorname{good}$,

(ii) $\left(B^{*}, b, \ldots, c, \ldots\right)_{c \in B} \equiv_{l^{\prime}}\left(B^{\prime}, b^{\prime}, \ldots, c, \ldots\right)_{c \in B}$.

The parallel change in $2.13(2)$ (that is, defining simply ${ }^{\otimes} \operatorname{good}$ ) is

(B)' for every $k, l \in \mathbb{N}$ for some $k^{\otimes}=k^{\otimes}(k, l) \in \mathbb{N}$ we have: if $B<_{s} B^{*}$ and $|B| \leq l$, then $\left(B^{*}, B, B^{*}, k, k^{\otimes}\right)$ is simply good.

This does not change the conclusions, i.e. 2.13, 2.17, 2.18, 2.19.

(2) We can change Definition 2.9 as we have changed Definition 2.13(1) in $2.13(3)$ and/or in 2.14(1).

(3) We can demand in 2.13(1)(A) that $m^{*}\left(k, l, l^{\prime}\right)=1$ at the expense of increasing $k^{*}$, because if $\mathrm{cl}^{k^{* *}}(\bar{a}, M) \supseteq \mathrm{cl}^{k^{*}, m^{*}}(\bar{a}, M)$ whenever $\bar{a} \in{ }^{l}|M|$, $M \in \mathcal{K}$ then $k^{* *}$ will do.

(4) In Definition 2.13 we can omit $m^{*}$ if $\mathrm{cl}$ is transparent by increasing $k^{*}$ (that is, $m^{*}=1$ ).

Lemma 2.15 below (the addition theorem, see $[\mathrm{CK}]$ or $[\mathrm{Gu}]$ and see more [Sh 463]) is an immediate corollary of the well known addition theorem; this is the point where $U$ is used.

LEMMA 2.15. For a finite vocabulary $\tau$ and f.o. formula $\psi\left(\bar{z}, \bar{z}^{1}, \bar{z}^{2}\right)$ (in $\tau), \bar{z}=\left\langle z_{1}, \ldots, z_{s}\right\rangle$, there are $i^{*} \in \mathbb{N}$ and $\tau$-formulas $\theta_{i}^{1}\left(\bar{z}, \bar{z}^{1}\right)=\theta_{i, \psi}^{1}\left(\bar{z}, \bar{z}^{1}\right)$, $\theta_{i}^{2}\left(\bar{z}, \bar{z}^{2}\right)=\theta_{i, \psi}^{2}\left(\bar{z}, \bar{z}^{2}\right)$ for $i<i^{*}$, each of quantifier depth at most that of $\psi$, such that: if $N$ is a $\tau$-model,

$$
N_{1} \underset{N_{0}}{N} N_{2}, \quad N_{1} \cap N_{2}=N_{0}, \quad N_{1} \cup N_{2}=N
$$

and the set of elements of $N_{0}$ is $\left\{c_{1}, \ldots, c_{s}\right\}, \bar{c}=\left\langle c_{1}, \ldots, c_{s}\right\rangle$ with $s=\lg (\bar{z})$ and $\bar{c}^{1} \in \lg \left(\bar{z}^{1}\right)\left(N_{1}\right)$ and $\bar{c}^{2} \in \lg \left(\bar{z}^{2}\right)\left(N_{2}\right)$ then

$N=\psi\left[\bar{c}, \bar{c}^{1}, \bar{c}^{2}\right] \quad$ iff $\quad$ for some $i<i^{*}, N_{1}=\theta_{i}^{1}\left[\bar{c}, \bar{c}^{1}\right]$ and $N_{2}=\theta_{i}^{2}\left[\bar{c}, \bar{c}^{2}\right]$.

Main Lemma 2.16 (Context as above). Assume ( $\mathfrak{K}, \mathrm{cl}$ ) is almost nice and $\mathrm{cl}$ is f.o. definable.

(1) Let $\varphi(\bar{x})$ be a f.o. formula in the vocabulary $\tau_{\mathcal{K}}$. Then for some $m_{\varphi} \in$ $\mathbb{N}$ and $k=k_{\varphi} \geq \lg (\bar{x})+$ q.d. $(\varphi(\bar{x}))\left({ }^{3}\right)$ and for some f.o. $\psi_{\varphi}(\bar{x})$ we have:

$(*)_{\varphi}$ for every random enough $\mathcal{M}_{n}$ and $\bar{a} \in \lg (\bar{x})\left|\mathcal{M}_{n}\right|$ we have

$$
(* *) \mathcal{M}_{n}=\varphi(\bar{a}) \text { if and only if } \mathcal{M}_{n}\left\lceil\mathrm{cl}^{k_{\varphi}, m_{\varphi}}\left(\bar{a}, \mathcal{M}_{n}\right) \models \psi_{\varphi}(\bar{a})\right. \text {. }
$$

(2) Moreover, if for simplicity we will consider " $y \in \mathrm{cl}^{k, m}(\bar{x}, M)$ " as an atomic formula when computing the q.d. of $\psi_{\varphi}$ (and so can omit the assump-

$\left({ }^{3}\right)$ q.d. stands for quantifier depth. 
tion "cl is f.o. definable") then we can demand: the number of alternations of quantifiers of $\psi_{\varphi}$ is $\leq$ those of $\varphi$, more precisely if $\varphi$ is $\Pi_{n}\left(\right.$ or $\left.\Sigma_{n}\right)$ then so is $\psi_{\varphi}$.

Proof. We shall ignore (2) (which is not used and is obvious if we understand the proof below). We prove the statement in (1) by induction on $r=$ q.d. $(\varphi(\bar{x}))$ and first note (by clause (e) of Definition 2.2 as " $y \in \mathrm{cl}^{k, m}(\bar{x})$ " is f.o. definable in $\mathcal{K})$ that $(*)_{\varphi}$ implies

$(*)_{\varphi}^{+}$in $(*)_{\varphi}$, possibly changing $\psi_{\varphi}$, one can replace $\mathcal{M}_{n}\left\lceil\mathrm{cl}^{k_{\varphi}, m_{\varphi}}\left(\bar{a}, \mathcal{M}_{n}\right)\right.$ by any $N$ with $\mathrm{cl}^{k_{\varphi}, m_{\varphi}}\left(\bar{a}, \mathcal{M}_{n}\right) \subseteq N \subseteq \mathcal{M}_{n}$.

Case 1: $\varphi$ atomic. Trivial [Proof: If $\varphi(\bar{x})$ is an atomic formula we let $m_{\varphi}=0, k_{\varphi}=0$ or whatever. So $\mathrm{cl}^{k_{\varphi}, m_{\varphi}}\left(\bar{a}, \mathcal{M}_{n}\right)=\bar{a}$ for our $k_{\varphi}, m_{\varphi}$. Assume $\mathcal{M}_{n}=\varphi(\bar{a})$ and let $\psi_{\varphi}=\varphi$. Now as $\bar{a} \subseteq \mathcal{M}_{n}\left\lceil\operatorname{cl}^{k_{\varphi}, m_{\varphi}}\left(\bar{a}, \mathcal{M}_{n}\right) \subseteq \mathcal{M}_{n}\right.$ we have $\mathcal{M}_{n} \models \varphi(\bar{a})$ iff $\mathrm{cl}^{k_{\varphi}, m_{\varphi}}\left(\bar{a}, \mathcal{M}_{n}\right) \models \psi_{\varphi}(\bar{a})$ as required.]

Case 2: $\varphi$ a Boolean combination of atomic formulas and formulas of the form $(\exists x) \varphi^{\prime}(x, \bar{y})$ with q.d. $\left(\varphi^{\prime}\right)<r$. Clearly follows by cases 3 and 1 .

Case 3: $r>0$ and $\varphi(\bar{x})=(\exists y) \varphi_{1}(\bar{x}, y)$. Let

$$
\begin{aligned}
& (*)_{1} m^{*}=m^{*}\left(k_{\varphi_{1}}, m_{\varphi_{1}}, \lg (\bar{x}), l^{\prime}\right), \quad k_{\varphi}=k^{*}\left(k_{\varphi_{1}}, m_{\varphi_{1}}, \lg (\bar{x}), l^{\prime}\right), \\
& \quad t=t\left(k_{\varphi_{1}}, m_{\varphi_{1}}, \lg (\bar{x}), l^{\prime}\right)
\end{aligned}
$$

be as guaranteed in Definition 2.9 with $l^{\prime}$ suitable (see its use below $\left({ }^{4}\right)$ ) and let $m_{\varphi}:=m^{*}+m_{\varphi_{1}}$. Let $\psi_{\varphi_{1}}^{1}$ witness $(*)_{\varphi_{1}}$, and let $\psi_{\varphi_{1}}^{2}$ witness $(*)_{\varphi_{1}}$.

So it is enough to prove the following two statements:

Statement 1. There is $\psi_{\varphi}^{1}(\bar{x})$ (f.o.) such that:

$(\otimes)_{1}$ for every random enough $\mathcal{M}_{n}$ and for every $\bar{a} \in \lg (\bar{x})\left|\mathcal{M}_{n}\right|$ we have $(\alpha)_{1} \Leftrightarrow(\beta)_{1}$ where:

$(\alpha)_{1} \mathcal{M}_{n}\left\lceil\mathrm{cl}^{k_{\varphi}, m_{\varphi}}\left(\bar{a}, \mathcal{M}_{n}\right) \models \psi_{\varphi}^{1}(\bar{a})\right.$,

$(\beta)_{1} \mathcal{M}_{n}=$ "there is $b \in \mathrm{cl}^{k_{\varphi}, m^{*}}\left(\bar{a}, \mathcal{M}_{n}\right)$ such that $\varphi_{1}(\bar{a}, b)$ holds"

(i.e. $b$ belongs to a small enough neighbourhood of $\bar{a})$.

STATEMENT 2. There is $\psi_{\varphi}^{2}(\bar{x})$ (f.o.) such that:

$(\bigotimes)_{2}$ for every random enough $\mathcal{M}_{n}$ and for every $\bar{a} \in \lg (\bar{x})\left|\mathcal{M}_{n}\right|$ we have $(\alpha)_{2} \Leftrightarrow(\beta)_{2}$ where:

$(\alpha)_{2} \mathcal{M}_{n}\left\lceil\mathrm{cl}^{k, m^{*}}\left(\bar{a}, \mathcal{M}_{n}\right) \models \psi_{\varphi}^{2}(\bar{a})\right.$,

$(\beta)_{2} \mathcal{M}_{n} \models$ "there is $b \in \mathcal{M}_{n} \backslash \operatorname{cl}^{k_{\varphi}, m^{*}}\left(\bar{a}, \mathcal{M}_{n}\right)$ such that $\varphi_{1}(\bar{a}, b)$ holds" (i.e. $b$ is far from $\bar{a})$.

(Note: $(\beta)_{1},(\beta)_{2}$ are complementary, but it is enough that always at least one of them holds.)

$\left({ }^{4}\right)$ Recall that $l^{\prime}$ should be larger than the quantifier depth of the formulas used, not their length, so the use of $t$ is O.K. 
Note that as " $y \in \mathrm{cl}^{k_{\varphi}, m^{*}}(\bar{x})$ " is f.o. definable and $m_{\varphi}=m^{*}+m_{\varphi_{1}} \geq m^{*}$, by clause (e) from 2.2 , we can in $(\alpha)_{2}$ replace $m^{*}$ by $m_{\varphi}$, changing $\psi_{\varphi}^{2}$ to $\psi_{\varphi}^{2.5}$.

Clearly these two statements are enough and $\psi_{\varphi}^{1}(\bar{x}) \vee \psi_{\varphi}^{2.5}(\bar{x})$ is as required.

Proof of Statement 1. Easy, recalling that $k_{\varphi} \geq k_{\varphi_{1}}$ by clause (A) of Definition 2.9, by the induction hypothesis as

$$
\mathrm{cl}^{k_{\varphi_{1}}, m_{\varphi_{1}}}\left(\bar{a} b, M_{n}\right) \subseteq \mathrm{cl}^{k_{\varphi}, m^{*}+m_{\varphi_{1}}}\left(\bar{a}, \mathcal{M}_{n}\right)=\operatorname{cl}^{k_{\varphi}, m_{\varphi}}\left(\bar{a}, \mathcal{M}_{n}\right)
$$

and by the fact that the closure is sufficiently definable.

Proof of Statement 2. We will use a series of equivalent statements $\otimes_{l}$.

$\otimes_{1}$ is $(\beta)_{2}$.

$\otimes_{2}$ There are $m_{2} \in\left[m_{\varphi_{1}}, m^{*}\right], m_{1} \leq m^{*}-m_{2}, b, B$ and $B^{*}, B^{\prime}$ such that:
( $\alpha) b \in \mathcal{M}_{n}, b \notin \mathrm{cl}^{k_{\varphi}, m^{*}}\left(\bar{a}, \mathcal{M}_{n}\right), \bar{a} \subseteq B \subseteq \operatorname{cl}^{k_{\varphi_{1}}, m_{1}}\left(\bar{a}, \mathcal{M}_{n}\right),|B| \leq t$,
( $\beta) B^{*}=B \cup\left[\mathrm{cl}^{k_{\varphi_{1}}, m_{\varphi_{1}}}\left(\bar{a} b, \mathcal{M}_{n}\right) \backslash \mathrm{cl}^{k_{\varphi_{1}}, m_{2}}\left(B, \mathcal{M}_{n}\right)\right]$ [hence $B=B^{*} \cap$ $\left.\mathrm{cl}^{k_{\varphi_{1}}, m_{2}}\left(B, \mathcal{M}_{n}\right)\right]$
( $\gamma) B \leq_{s} \quad B^{\prime} \in \mathcal{K}_{\infty}$ and $B^{\prime}=B^{*}$ or at least $\left(B^{*}, b, c\right)_{c \in B} \equiv_{l^{\prime}}$ $\left(B^{\prime}, b, c\right)_{c \in B}($ see $2.11(4))$,

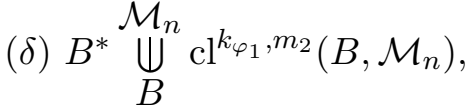
(ع) $\left(B^{\prime}, B, \bar{a} b, k, m_{0}, m_{2}\right)$ is good,

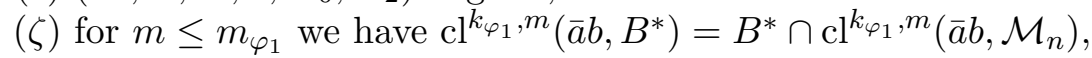
$\oplus_{2} \mathcal{M}_{n}=\varphi_{1}(\bar{a}, b)$.

Then we have

$$
(*)_{2} \otimes_{1} \Leftrightarrow \otimes_{2} .
$$

Why? The implication $\Leftarrow$ is trivial as $\oplus_{1}$ is included in $\otimes_{2}$, the implication $\Rightarrow$ holds by clause (A) in the definition of almost nice 2.9, except $b \notin \mathrm{cl}^{k_{\varphi}, m^{*}}\left(\bar{a}, \mathcal{M}_{n}\right)$ which is explicitly demanded in $(\beta)_{2}$.

Next, let

$\otimes_{3}$ like $\otimes_{2}$ but with $\oplus_{2}$ replaced by

$$
\oplus_{3} \mathcal{M}_{n}\left\lceil\mathrm{cl}^{k_{\varphi_{1}}, m_{\varphi_{1}}\left(\bar{a} b, \mathcal{M}_{n}\right) \models} \psi_{\varphi_{1}}^{1}(\bar{a}, b) .\right.
$$

Then

$$
(*)_{3} \otimes_{2} \Leftrightarrow \otimes_{3} .
$$

Why? By the induction hypothesis.

Next, let

$\otimes_{4}$ like $\otimes_{3}$ but with $\oplus_{3}$ replaced by

$$
\oplus_{4} \mathcal{M}_{n} \uparrow\left[B^{*} \cup \mathrm{cl}^{k_{\varphi_{1}}, m_{2}}\left(B, \mathcal{M}_{n}\right)\right] \models \psi_{\varphi_{1}}^{2}(\bar{a}, b) .
$$


Again, we have

$$
(*)_{4} \otimes_{3} \Leftrightarrow \otimes_{4} .
$$

Why? By $(*)_{\varphi_{1}}^{+}$at the beginning of the proof, the definition of $B^{*}$ and the choice of $\psi_{\varphi_{1}}^{2}$. (Let $\otimes_{3}$ be true. Since by the choice of $B^{*}$ and $B$ above, $\operatorname{cl}^{k_{\varphi_{1}}, m_{\varphi_{1}}}\left(\bar{a} b, \mathcal{M}_{n}\right) \subseteq \operatorname{cl}^{k_{\varphi_{1}}, m_{\varphi_{1}}}\left(\bar{a} b, \mathcal{M}_{n}\right) \cup \operatorname{cl}^{k_{\varphi_{1}}, m_{2}}\left(B, \mathcal{M}_{n}\right)=B^{*} \cup$ $\mathrm{cl}^{k_{\varphi_{1}}, m_{2}}\left(B, \mathcal{M}_{n}\right) \subseteq \mathcal{M}_{n}$ we have $\mathcal{M}_{n} \models \varphi_{1}(\bar{a}, b)$ iff $B^{*} \cup \operatorname{cl}^{k_{\varphi_{1}}, m_{2}}\left(B, \mathcal{M}_{n}\right) \models$ $\psi_{\varphi_{1}}^{2}(\bar{a} b)$ by $(*)_{\varphi_{1}}^{+}$. So $(*)_{4}$ holds. $)$

For notational simplicity we assume $B \neq \emptyset$, and similarly assume $\bar{a}$ is with no repetition; we shall apply Lemma 2.15 several times.

First for $m \leq m_{\varphi_{1}}$ we apply 2.15 to the case $s=t, \bar{z}=\left\langle z_{1}, \ldots, z_{t}\right\rangle, \bar{z}^{1}=$ $\left\langle z_{1}^{1}, z_{2}^{1}\right\rangle, \bar{z}^{2}$ empty and the formula " $z_{2}^{1} \in \mathrm{cl}^{k_{\varphi_{1}}, m}\left(\bar{z}, z_{1}^{1}\right)$ " and get $i_{1, m}^{*} \in \mathbb{N}$ and formulas $\theta_{1, m, i}^{1}\left(\bar{z}, z_{1}^{1}, z_{2}^{1}\right)$ and $\theta_{1, m, i}^{2}(\bar{z})$ for $i<i_{1, m}^{*}$. Let

$$
u_{1}^{*}=\left\{(m, i): m \leq m_{\varphi_{1}}, i<i_{1, m}^{*}\right\} .
$$

Second for $m \leq m_{\varphi_{1}}$ we apply 2.15 to the case $s=t, \bar{z}^{2}=\left\langle z_{1}^{2}\right\rangle, \bar{z}^{1}=\left\langle z_{1}^{1}\right\rangle$, $\bar{z}=\left\langle z_{1}, \ldots, z_{t}\right\rangle$ and the formula " $z_{1}^{2} \in \mathrm{cl}^{k_{\varphi_{1}}, m}\left(\bar{z}, z_{1}^{1}\right)$ " and get $i_{2, m}^{*} \in \mathbb{N}$ and formulas $\theta_{2, m, i}^{1}\left(\bar{z}, \bar{z}^{1}\right)$ and $\theta_{2, m, i}^{2}\left(\bar{z}, z_{1}^{2}\right)$ for $i<i_{2, m}^{*}$.

Let $\tau^{\prime}=\tau_{\mathcal{K}} \cup\left\{P_{1}, P_{2}\right\}$, with $P_{1}, P_{2}$ new unary predicates: for $\theta \in \mathcal{L}\left[\tau_{\mathcal{K}}^{\prime}\right]$ let $\theta^{\left[P_{l}\right]}$ be $\theta$ with the quantifiers restricted to $P_{l}$. Let $\psi^{*}=\psi_{1}^{*} \wedge \psi_{2}^{*} \wedge \psi_{3}^{*}$ where

$$
\begin{aligned}
& \psi_{1}^{*}:=\psi_{\varphi_{1}}^{2}\left(z_{1}, \ldots, z_{\lg (\bar{x})}, z_{1}^{1}\right) \\
& \psi_{2}^{*}:=\bigwedge_{m \leq m_{\varphi_{1}}}(\forall y)\left[y \in \mathrm{cl}^{k_{\varphi_{1}}, m}\left(\left\{z_{1}, \ldots, z_{\lg (\bar{x})}, z_{1}^{1}\right\}\right)\right. \\
&\left.\quad \equiv\left(\psi_{2, m}^{*, 1}\left(z_{1}, \ldots, z_{t}, z_{1}^{1}, y\right) \vee \psi_{2, m}^{*, 2}\left(z_{1}, \ldots, z_{t}, z_{1}^{1}, y\right)\right)\right],
\end{aligned}
$$

where

$$
\begin{aligned}
\psi_{2, m}^{*, 1}\left(z_{1}, \ldots, z_{t}, z_{1}^{1}, y\right) & \\
& :=\bigvee_{i<i_{1, m}^{*}}\left(\theta_{1, m, i}^{1}\left(z_{1}, \ldots, z_{t}, z_{1}^{1}, y\right)^{\left[P_{1}\right]} \wedge \theta_{1, m, i}^{2}\left(z_{1}, \ldots, z_{t}\right)^{\left[P_{2}\right]}\right), \\
\psi_{2, m}^{*, 2}\left(z_{1}, \ldots, z_{t}, z_{1}^{1}, y\right) & :=\bigvee_{i<i_{2, m}^{*}}\left(\theta_{2, m, i}^{1}\left(z_{1}, \ldots, z_{t}, z_{1}^{1}\right)^{\left[P_{1}\right]} \wedge \theta_{2, m, i}^{2}\left(z_{1}, \ldots, z_{t}, y\right)^{\left[P_{2}\right]}\right),
\end{aligned}
$$

and let

$$
\begin{aligned}
\psi_{3}^{*}:=(\forall y)\left(P _ { 1 } ( y ) \equiv \left[\bigvee_{l=1}^{t} y=z_{l} \vee\right.\right. \\
\left.\quad\left(y \in \mathrm{cl}^{k_{\varphi_{1}}, m_{\varphi_{1}}}\left(\left\{z_{1}, \ldots, z_{\lg (\bar{x})}, z_{1}^{1}\right\} \wedge y \notin \operatorname{cl}^{k_{\varphi_{1}}, m_{2}}\left\{z_{1}, \ldots, z_{\lg (\bar{x})}\right\}\right)\right]\right) .
\end{aligned}
$$

So we have defined $\psi^{*}$. Now we apply 2.15 the third time, with the vocabulary $\tau_{\mathcal{K}} \cup\left\{P_{1}, P_{2}\right\}$, to the case $s=t, \bar{z}^{2}$ empty, $\bar{z}^{1}=\left\langle z_{1}^{1}\right\rangle, \bar{z}=$ 
$\left\langle z_{1}, \ldots, z_{\lg (\bar{x})}\right\rangle$, and $\psi\left(\bar{z}, \bar{z}^{1}, \bar{z}^{2}\right)=\psi\left(\bar{z}, z_{1}^{1}\right)=\psi^{*}\left(\left\langle z_{1}, \ldots, z_{\lg (\bar{x})}\right\rangle, z_{1}^{1}\right)$ and get $i^{*}, \theta_{3, i}^{1}\left(\bar{z}, \bar{z}^{1}\right)$ and $\theta_{3, i}^{2}\left(\bar{z}, \bar{z}^{2}\right)$ for $i<i^{*}$ as there. Let

$\otimes_{5}$ like $\otimes_{4}$ but with $\oplus_{4}$ replaced by

$\oplus_{5}$ letting $c_{1}, \ldots, c_{t}$ list $B$ possibly with repetitions but such that $\left\langle c_{1}, \ldots, c_{\lg (\bar{x})}\right\rangle=\bar{a}$ and letting

$$
P_{1}^{*}=B^{*} \quad \text { and } \quad P_{2}^{*}=\operatorname{cl}^{k_{\varphi_{1}}, m_{2}}\left(\left\{c_{1}, \ldots, c_{t}\right\}, \mathcal{M}_{n}\right)
$$

we have

(*) $\left(\mathcal{M}_{n}\left\lceil\left(P_{1}^{*} \cup P_{2}^{*}\right), P_{1}^{*}, P_{2}^{*}\right) \models \psi^{*}\left[c_{1}, \ldots, c_{t}, b\right]\right.$ (the model is a $\tau^{\prime}$-model).

Now

$(*)_{5} \otimes_{4} \Leftrightarrow \otimes_{5}$.

Why? Look at what the statements mean recalling

$$
\mathcal{M}_{n}\left\lceilP _ { 1 } ^ { * } \underset { B } { \bigcup _ { B } ^ { \mathcal { M } } } \mathcal { M } _ { n } \left\lceil P_{2}^{*} .\right.\right.
$$

Next let

$\otimes_{6}$ like $\otimes_{5}$ but with $\oplus_{5}$ replaced by

$\oplus_{6}$ letting $c_{1}, \ldots, c_{t}$ list $B$ possibly with repetitions but such that $\left\langle c_{1}, \ldots, c_{\lg (\bar{x})}\right\rangle=\bar{a}$ and letting

$$
P_{1}^{*}=B^{*} \quad \text { and } \quad P_{2}^{*}=\mathrm{cl}^{k_{\varphi_{1}}, m_{2}}\left(\left\{c_{1}, \ldots, c_{t}\right\}, \mathcal{M}_{n}\right)
$$

there is $i<i^{*}$ such that:

(i) $\left(\mathcal{M}_{n}\left\lceil P_{1}^{*}, P_{1}^{*}, P_{2}^{*} \cap P_{1}^{*}\right) \models \theta_{3, i}^{1}\left[\left\langle c_{1}, \ldots, c_{t}\right\rangle, b\right]\right.$,

(ii) $\left(\mathcal{M}_{n}\left\lceil P_{2}^{*}, P_{1}^{*} \cap P_{2}^{*}, P_{2}^{*}\right) \models \theta_{3, i}^{2}\left[\left\langle c_{1}, \ldots, c_{t}\right\rangle\right]\right.$.

Now

$$
(*)_{6} \otimes_{5} \Leftrightarrow \otimes_{6} .
$$

Why? By the choice of $\theta_{3, i}^{1}, \theta_{3, i}^{2}\left(i<i^{*}\right)$.

However in the two $\tau^{\prime}$-models appearing in $\oplus_{6}$, the predicates $P_{1}, P_{2}$ are interpreted in a trivial way: as the whole universe of the model or as $\left\{c_{1}, \ldots, c_{t}\right\}$.

So let:

(a) $\theta_{4, i}^{1}\left(z_{1}, \ldots, z_{t}, y\right)$ be $\theta_{3, i}^{1}\left(z_{1}, \ldots, z_{t}, y\right)$ with each atomic formula of the form $P_{1}(\sigma)$ or $P_{2}(\sigma)$ replaced by $\sigma=\sigma$ or $\bigvee_{r=1}^{t} \sigma=z_{r}$ respectively,

(b) $\theta_{4, i}^{2}\left(z_{1}, \ldots, z_{t}\right)$ be $\theta_{3, i}^{2}\left(z_{1}, \ldots, z_{t}\right)$ with each atomic formula of the form $P_{1}(\sigma)$ or $P_{2}(\sigma)$ replaced by $\bigvee_{r=1}^{t} \sigma=z_{r}$ or $\sigma=\sigma$ respectively. 
Then let (recall $B^{\prime}$ is mentioned in $\otimes_{2}$, a "replacement" for $B^{*}$ )

$\otimes_{7}$ like $\otimes_{6}$ but with $\oplus_{6}$ replaced by

$\oplus_{7}$ letting $c_{1}, \ldots, c_{t}$ list $B$ possibly with repetitions but such that $\left\langle c_{1}, \ldots, c_{\lg (\bar{x})}\right\rangle=\bar{a}$, there is $i<i^{*}$ such that

(i) $\mathcal{M}_{n}\left\lceil B^{\prime} \models \theta_{4, i}^{1}\left[\left\langle c_{1}, \ldots, c_{t}\right\rangle, b\right]\right.$ and

(ii) $\mathcal{M}_{n}\left\lceil\mathrm{cl}^{k_{\varphi_{1}}, m_{2}}\left(\left\langle c_{1}, \ldots, c_{t}\right\rangle, \mathcal{M}_{n}\right) \models \theta_{4, i}^{2}\left(\left\langle c_{1}, \ldots, c_{t}\right\rangle\right)\right.$.

We have

$$
(*)_{7} \otimes_{6} \Leftrightarrow \otimes_{7} .
$$

Why? By the choice of the $\theta_{4, i}^{1}, \theta_{4, i}^{2}$ and the property of $B^{\prime}$ (stated in $\otimes_{2}$ ).

Let $\mathcal{P}=\left\{\left(N, c_{1}, \ldots, c_{t}\right): N \in \mathcal{K}_{\infty}\right.$, with the set of elements $\left.\left\{c_{1}, \ldots, c_{t}\right\}\right\}$. Let $\left\{\left(N_{j}, c_{1}^{j}, \ldots, c_{t}^{j}\right): j<j^{*}\right\}$ list the members of $\mathcal{P}$ up to isomorphism, so with no two isomorphic. For every $j<j^{*}$ and $i<i^{*}$ choose if possible $\left(N_{j, i}, c_{1}^{j}, \ldots, c_{t}^{j}, b_{i}^{j}\right)$ such that:

(i) $N_{j} \leq_{s} N_{j, i}\left(\right.$ in $\left.\mathcal{K}_{\infty}\right)$,

(ii) $b_{i}^{j} \in N_{j, i} \backslash N_{j}$,

(iii) $N_{j, i}=\theta_{4, i}^{1}\left(\left\langle c_{1}^{j}, \ldots, c_{t}^{j}\right\rangle, b_{i}^{j}\right)$,

(iv) $\left(N_{j, i}, B,\left\{c_{i}^{j}: i=1, \ldots, \lg (\bar{x})\right\} \cup\left\{b_{i}^{j}\right\}, k, m_{0}, m_{2}\right)$ is good for $\mathfrak{K}$.

Let

$w=\left\{(i, j): i<i^{*}, j<j^{*}\right.$ and $\left(N_{j, i}, c_{1}^{j}, \ldots, c_{t}^{j}, b_{i}^{j}\right)$ is well defined $\}$.

Let

$\otimes_{8}$ There are $m_{2} \leq m^{*}, m_{1} \leq m^{*}-m_{2}$ such that $m_{2} \geq m_{\varphi_{1}}$, and there are $b, B$ such that:

$$
\bar{a} \subseteq B \subseteq \mathrm{cl}^{k^{*}, m_{2}}\left(\bar{a}, \mathcal{M}_{n}\right),|B| \leq t\left(k_{\varphi_{1}}, m_{\varphi_{1}}, \lg (\bar{x})\right), b \notin \mathrm{cl}^{k^{*}, m^{*}}\left(\bar{a}, \mathcal{M}_{n}\right),
$$

$b \in \mathcal{M}_{n}$, and

$\oplus_{8}$ for some $c_{1}, \ldots, c_{t}$ listing $B$ such that $\bar{a}=\left\langle c_{1}, \ldots, c_{\lg (\bar{x})}\right\rangle$ there are $i<i^{*}, j<j^{*}$ such that $(i, j) \in w$ and:

(i) $\left(\mathcal{M}_{n}\left\lceil B, c_{1}, \ldots, c_{t}\right) \cong\left(N_{j}, c_{1}^{j}, \ldots, c_{t}^{j}\right)\right.$, i.e. the mapping $c_{1}^{j} \mapsto c_{1}$, $c_{2}^{j} \mapsto c_{2}$ embeds $N_{j}$ into $\mathcal{M}_{n}$

(ii) $\mathcal{M}_{n}\left\lceil\mathrm{cl}^{k_{\varphi_{1}}, m_{2}}\left(B, \mathcal{M}_{n}\right) \models \theta_{4, i}^{2}\left(\left\langle c_{1}, \ldots, c_{t}\right\rangle\right)\right.$.

Then

$$
(*)_{8} \otimes_{7} \Leftrightarrow \otimes_{8} .
$$

Why? To prove $\otimes_{7} \Rightarrow \otimes_{8}$ let $c_{1}, \ldots, c_{t}$ as well as $i<i^{*}$ be as in $\oplus_{7}$, and let $j<j^{*}$ be such that $\left(\mathcal{M}_{n} \uparrow B, c_{1}, \ldots, c_{t}\right) \cong\left(N_{j}, c_{1}^{j}, \ldots, c_{t}^{j}\right)$. The main point is that $B^{\prime}$ exemplifies that $(i, j) \in w$. 
To prove $\otimes_{8} \Rightarrow \otimes_{7}$ use the definition of goodness in clause $(\varepsilon)$ (see $\otimes_{2}$ ) and Definition 2.8(4).

We have now finished as $\otimes_{8}$ can be expressed as a f.o. formula straightforwardly. So we have carried out the induction step on the quantifier depth, thus finishing the proof.

Lemma 2.17. (1) Assume $(\mathfrak{K}, \mathrm{cl})$ is simply almost nice and $\mathrm{cl}$ is f.o. definable. Let $\varphi(\bar{x})$ be a f.o. formula in the vocabulary $\tau_{\mathcal{K}}$. Then for some $k=k_{\varphi}$ and f.o. formula $\psi_{\varphi}(\bar{x})$ we have:

(*) for every random enough $\mathcal{M}_{n}$ and $\bar{a} \in{ }^{\lg (\bar{x})}\left|\mathcal{M}_{n}\right|$, $(* *) \mathcal{M}_{n} \models \varphi(\bar{a})$ if and only if $\mathcal{M}_{n}\left\lceil\mathrm{cl}^{k_{\varphi}}\left(\bar{a}, \mathcal{M}_{n}\right) \models \psi_{\varphi}(\bar{a})\right.$.

(2) The number of alternations of quantifiers of $\psi_{\varphi}$ in (1) is $\leq$ the number of alternations of quantifiers of $\varphi$ if we consider " $y \in \mathrm{cl}^{k, m}(\bar{x}, M)$ " as atomic (so we can omit the assumption "cl is f.o. definable"). More precisely, if $\varphi$ is $\Pi_{n}\left(\right.$ or $\left.\Sigma_{n}\right)$ then so is $\psi_{\varphi}$.

REMARK 2.18. (1) Of course we do not need to assume that the closure operation is definable, it is enough if there is a variant $\mathrm{cl}_{*}$ which is definable and for every $k, m$ there are $k^{1}, m^{1}, k^{2}, m^{2}$ such that always

$$
\mathrm{cl}^{k, m}(A, M) \subseteq \mathrm{cl}_{*}^{k^{1}, m^{1}}(A, M) \subseteq \mathrm{cl}^{k^{2}, m^{2}}(A, M) .
$$

(2) Similarly in 2.16 (using Definition 2.10).

(3) We can weaken "simply almost nice" as in Definition 2.14(1) and still part (1) is true, with essentially the same proof.

(4) The proof of 2.17 is somewhat simpler than the proof of 2.16 .

Proof. (1) We prove the statement by induction on $r=$ q.d. $(\varphi(\bar{x}))$. First note (by clause (e) of 2.2)

$(*)_{\varphi}^{+}$in $(*)$ (of 2.17) one can replace $\mathcal{M}_{n}\left\lceil\operatorname{cl}^{k_{\varphi}}\left(\bar{a}, \mathcal{M}_{n}\right)\right.$ by any $N$ with

$$
\operatorname{cl}^{k_{\varphi}}\left(\bar{a}, \mathcal{M}_{n}\right) \subseteq N \subseteq \mathcal{M}_{n}
$$

(possibly changing $\psi_{\varphi}$ ).

Case 1: $\varphi$ atomic. Trivial.

Case 2: $\varphi$ a Boolean combination of atomic formulas and formulas of quantifier depth $\leq r$. Clearly follows by cases 3 and 1 . Trivial.

Case 3: $r>0$ and $\varphi(\bar{x})=(\exists y) \varphi_{1}(\bar{x}, y)$. Let (the functions are from $2.13(1))$

$$
m^{*}=m^{*}\left(k_{\varphi_{1}}, \lg (\bar{x}), l^{\prime}\right), \quad k^{*}=k^{*}\left(k_{\varphi_{1}}, \lg (\bar{x}), l^{\prime}\right), \quad t=t\left(k_{\varphi_{1}}, \lg (\bar{x}), l^{\prime}\right)
$$

with $l^{\prime}$ suitable (just the quantifier depth of $\psi_{\varphi_{1}}^{2}$ defined below) and let $k_{\varphi}$ 
be $\left({ }^{5}\right)$ such that:

$(*)_{1}|A| \leq \lg (\bar{x})+1 \& A \subseteq N \in \mathcal{K} \Rightarrow \operatorname{cl}^{k_{\varphi_{1}}}\left(\mathrm{cl}^{k^{*}, m^{*}}(A, N), N\right) \subseteq \operatorname{cl}^{k_{\varphi}}(A, N)$. Let $\psi_{\varphi_{1}}^{1}(\bar{x}, y)$ witness $(*)_{\varphi_{1}}$, and let $\psi_{\varphi_{1}}^{2}(\bar{x}, y)$ witness $(*)_{\varphi_{1}}^{+}$.

It is enough to prove the following two statements (see below):

Statement 1. There is $\psi_{\varphi}^{1}(\bar{x})$ (f.o.) such that:

$(\bigotimes)_{1}$ for every random enough $\mathcal{M}_{n}$ and for every $\bar{a} \in \lg (\bar{x})\left|\mathcal{M}_{n}\right|$ we have $(\alpha)_{1} \Leftrightarrow(\beta)_{1}$ where:

$(\alpha)_{1} \mathcal{M}_{n}\left\lceil\operatorname{cl}^{k_{\varphi}}\left(\bar{a}, \mathcal{M}_{n}\right) \models \psi_{\varphi}^{1}(\bar{a})\right.$,

$(\beta)_{1} \mathcal{M}_{n}=$ "there is $b \in \mathrm{cl}^{k^{*}, m^{*}}\left(\bar{a}, \mathcal{M}_{n}\right)$ such that $\varphi_{1}(\bar{a}, b)$ holds."

Statement 2. There is $\psi_{\varphi}^{2}(\bar{x})$ (f.o.) such that:

$(\bigotimes)_{2}$ for every random enough $\mathcal{M}_{n}$ and for every $\bar{a} \in \lg (\bar{x})\left|\mathcal{M}_{n}\right|$ we have $(\alpha)_{2} \Leftrightarrow(\beta)_{2}$ where:

$(\alpha)_{2} \mathcal{M}_{n}\left\lceil\mathrm{cl}^{k^{*}, m^{*}}\left(\bar{a}, \mathcal{M}_{n}\right) \models \psi_{\varphi}^{2}(\bar{a})\right.$,

$(\beta)_{2} \mathcal{M}_{n} \models$ "there is $b \in \mathcal{M}_{n} \backslash \mathrm{cl}^{k^{*}, m^{*}}\left(\bar{a}, \mathcal{M}_{n}\right)$ such that $\varphi_{1}(\bar{a}, b)$ holds".

(Note: $(\beta)_{1},(\beta)_{2}$ are complementary, but it is enough that always at least one holds.)

Note that as " $y \in \mathrm{cl}^{k^{*}, m^{*}}(\bar{x})$ " is f.o. definable, by $2.2(\mathrm{e})$ and the choice of $k_{\varphi}$ we can in $(\alpha)_{2}$ replace $\mathrm{cl}^{k^{*}, m^{*}}$ by $\mathrm{cl}^{k_{\varphi}}$, changing $\psi_{\varphi}^{2}$ to $\psi_{\varphi}^{2.5}$ (just as from $(*)_{\psi}$ we have deduced $\left.(*)_{\varphi}^{+}\right)$.

Clearly these two statements are enough because if $\psi_{\varphi}^{1.5}$ expresses $(\alpha)_{1}$ then $\psi_{\varphi}^{1.5}(\bar{x}) \vee \psi_{\varphi}^{2.5}(\bar{x})$ is as required.

Proof of Statement 1. Easy, by the induction hypothesis as

$$
\mathrm{cl}^{k_{\varphi_{1}}}\left(\bar{a} b, M_{n}\right) \subseteq \operatorname{cl}^{k_{\varphi_{1}}}\left(\mathrm{cl}^{k^{*}, m^{*}}\left(\bar{a}, \mathcal{M}_{n}\right), \mathcal{M}_{n}\right) \subseteq \mathrm{cl}^{k_{\varphi}}\left(\bar{a}, \mathcal{M}_{n}\right)
$$

and by the fact that the closure is sufficiently definable. So in this case $\psi_{\varphi}^{1}(\bar{x})$ can be chosen as $(\exists y)\left[\psi_{\varphi_{1}}^{2}(\bar{x}, y) \wedge y \in \mathrm{cl}^{k^{*}, m^{*}}(\bar{x})\right]$.

Proof of Statement 2. We will use a series of equivalent statements $\otimes_{l}$.

$\otimes_{1}$ is $(\beta)_{2}$.

$\otimes_{2}$ There are $b, B$ and $B^{*}, B^{\prime}$ such that:

$\left({ }^{5}\right)$ If we change clause (A) of 2.13(1) a little, $k_{\varphi_{*}}=k^{*}$ will be O.K.: instead of assuming $b \notin \mathrm{cl}^{k^{*}, m^{*}}\left(\bar{a}, \mathcal{M}_{n}\right)$ assume just $\mathrm{cl}^{k}\left(\bar{a} b, \mathcal{M}_{n}\right) \nsubseteq \mathrm{cl}^{k^{*}, m^{*}}\left(\bar{a}, \mathcal{M}_{n}\right)$. Allowing $m^{*}$ to increase, the two versions are equivalent. Set $m^{* *}=m^{* *}\left(k, l, l^{\prime}\right)=m^{*}\left(k, l, l^{\prime}\right)+k$. Now by $2.4(3)$ we have $b \in \mathrm{cl}^{k^{*}, m^{*}}\left(\bar{a}, \mathcal{M}_{n}\right)$ and $c \in \operatorname{cl}^{k}\left(\bar{a} b, \mathcal{M}_{n}\right) \Rightarrow c \in \operatorname{cl}^{m^{*}+k}\left(\bar{a}, \mathcal{M}_{n}\right)=\operatorname{cl}^{m^{* *}}\left(\bar{a}, \mathcal{M}_{n}\right)$, hence $b \in \operatorname{cl}^{k^{*}, m^{*}}\left(\bar{a}, \mathcal{M}_{n}\right) \Rightarrow \operatorname{cl}^{k}\left(\bar{a} b, \mathcal{M}_{n}\right) \subseteq \operatorname{cl}^{k, m^{* *}}\left(\bar{a}, \mathcal{M}_{n}\right)$, hence $\operatorname{cl}^{k}\left(\bar{a} b, \mathcal{M}_{n}\right) \nsubseteq$ $\mathrm{cl}^{k, m^{* *}}\left(\bar{a}, \mathcal{M}_{n}\right) \Rightarrow b \notin \mathrm{cl}^{k^{*}, m^{*}}\left(\bar{a}, \mathcal{M}_{n}\right)$, so our new assumption for $m^{* *}$ implies our old one for $m^{*}$. Of course our new assumption for $m^{*}$ implies the old one for $m^{*}$. See Section 3 where this is done. 
( $\alpha) b \in \mathcal{M}_{n}, b \notin \mathrm{cl}^{k^{*}, m^{*}}\left(\bar{a}, \mathcal{M}_{n}\right)$

( $\beta) \bar{a} \subseteq B \subseteq \mathrm{cl}^{k^{*}, m^{*}}\left(\bar{a}, \mathcal{M}_{n}\right)$, moreover $\mathrm{cl}^{k_{\varphi_{1}}}\left(B, \mathcal{M}_{n}\right) \subseteq \mathrm{cl}^{k^{*}, m^{*}}\left(\bar{a}, \mathcal{M}_{n}\right)$, and $|B| \leq t$,

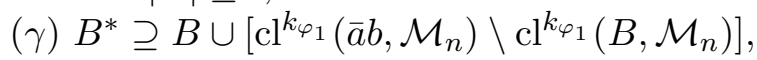

( $\delta) B \leq_{s} B^{\prime} \in \mathcal{K}_{\infty}$ and: $B^{\prime}=B$ or just $\left(B^{*}, b, c\right)_{c \in B} \equiv_{l^{\prime}}\left(B^{\prime}, b, c\right)_{c \in B}$ (see 2.11(4)),

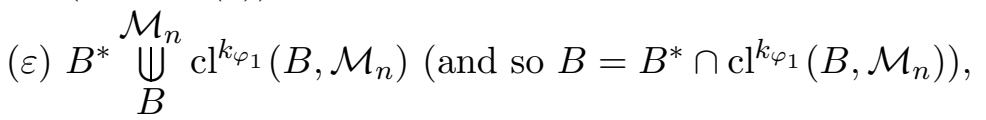

( $\zeta)\left(B^{\prime}, B, \bar{a} b, k\right)$ is simply good,

$(\eta) \operatorname{cl}^{k}\left(\bar{a} b, B^{*}\right) \backslash B=B^{*} \cap \operatorname{cl}^{k}\left(\bar{a} b, \mathcal{M}_{n}\right) \backslash \operatorname{cl}^{k}\left(B, \mathcal{M}_{n}\right)$, actually this follows from clauses $(\varepsilon),(\beta)$ if $\mathrm{cl}$ is "reasonable" $\left({ }^{6}\right)$,

$\oplus_{2} \mathcal{M}_{n}=\varphi_{1}(\bar{a}, b)$.

We have

$$
(*)_{2} \otimes_{1} \Leftrightarrow \otimes_{2} .
$$

Why? The implication $\Leftarrow$ is trivial as $\oplus_{1}$ is included in $\otimes_{2}$, the implication $\Rightarrow$ holds by clause (A) in Definition 2.13 of simply almost nice.

Next, let

$\otimes_{3}$ like $\otimes_{2}$ but with $\oplus_{2}$ replaced by

$$
\oplus_{3} \mathcal{M}_{n}\left\lceil\mathrm{cl}^{k_{\varphi_{1}}}\left(\bar{a} b, M_{n}\right) \models \psi_{\varphi_{1}}^{1}(\bar{a}, b) .\right.
$$

Then

$$
(*)_{3} \otimes_{2} \Leftrightarrow \otimes_{3} .
$$

Why? By the induction hypothesis and our choices.

Next, let

$\otimes_{4}$ like $\otimes_{3}$ but with $\oplus_{3}$ replaced by

$$
\oplus_{4} \mathcal{M}_{n} \uparrow\left[B^{*} \cup \mathrm{cl}^{k_{\varphi_{1}}}\left(B, \mathcal{M}_{n}\right)\right] \models \psi_{\varphi_{1}}^{2}(\bar{a}, b) .
$$

Then

$$
(*)_{4} \otimes_{3} \Leftrightarrow \otimes_{4} .
$$

Why? By $(*)_{\varphi_{1}}^{+}$at the beginning of the proof, the requirements on $B^{*}$ and the choice of $\psi_{\varphi_{1}}^{2}$.

For notational simplicity we assume $B \neq \emptyset$, and similarly assume $\bar{a}$ has no repetitions and apply Lemma 2.15 with the vocabulary $\tau_{\mathfrak{K}}$ to the case $s=t, \bar{z}^{2}$ empty, $\bar{z}^{1}=\left\langle z_{1}^{1}\right\rangle, \bar{z}=\left\langle z_{1}, \ldots, z_{t}\right\rangle$, and $\psi\left(\bar{z}, \bar{z}^{1}, \bar{z}^{2}\right)=\psi\left(\bar{z}, z_{1}^{1}\right)=$ $\psi_{\varphi_{1}}^{2}\left(\left\langle z_{1}, \ldots, z_{\lg (\bar{x})}\right\rangle, z_{1}^{1}\right)$ and get $i^{*}, \theta_{i}^{1}\left(\bar{z}, \bar{z}^{1}\right)$ and $\theta_{i}^{2}(\bar{z})$ for $i<i^{*}$ as there; in

\footnotetext{
$\left({ }^{6}\right)$ Which means: if $A \subseteq B \subseteq D, B \bigcup_{A}^{D} C, B^{\prime} \subseteq B$, then $\operatorname{cl}^{k}\left(B^{\prime}, B \cup C\right) \cap B=\operatorname{cl}^{k}\left(B^{\prime}, B\right)$.
} 
particular the quantifier depth of $\theta_{i}^{1}, \theta_{i}^{2}$ for $i<i^{*}$ is at most the quantifier depth of $\psi_{\varphi_{1}}^{2}$.

Next, let

$\otimes_{5}$ like $\otimes_{4}$ but with $\oplus_{4}$ replaced by

$\oplus_{5}$ letting $c_{1}, \ldots, c_{t}$ list $B$ possibly with repetitions but such that $\left\langle c_{1}, \ldots, c_{\lg (\bar{x})}\right\rangle=\bar{a}$, there is $i<i^{*}$ such that:

(i) $B^{*} \models \theta_{i}^{1}\left[\left\langle c_{1}, \ldots, c_{t}\right\rangle, b\right]$,

(ii) $\operatorname{cl}^{k}\left(B, \mathcal{M}_{n}\right) \models \theta_{i}^{2}\left[\left\langle c_{1}, \ldots, c_{t}\right\rangle\right]$.

Now

$(*)_{5} \otimes_{4} \Leftrightarrow \otimes_{5}$.

Why? By the choice of $\theta_{i}^{1}, \theta_{i}^{2}$ for $i<i^{*}$, so by Lemma 2.15.

Let $\mathcal{P}=\left\{\left(N, c_{1}, \ldots, c_{t}\right): N \in \mathcal{K}_{\infty}\right.$, with the set of elements $\left.\left\{c_{1}, \ldots, c_{t}\right\}\right\}$. Let $\left\{\left(N_{j}, c_{1}^{j}, \ldots, c_{t}^{j}\right): j<j^{*}\right\}$ list the members of $\mathcal{P}$ up to isomorphism, so with no two isomorphic. For every $j<j^{*}$ and $i<i^{*}$ choose if possible $\left(N_{j, i}, c_{1}^{j}, \ldots, c_{t}^{j}, b_{i}^{j}\right)$ such that:

(i) $N_{j} \leq_{s} N_{j, i}\left(\right.$ in $\left.\mathcal{K}_{\infty}\right)$,

(ii) $b_{i}^{j} \in N_{j, i} \backslash N_{j}$,

(iii) $N_{j, i} \mid=\theta_{i}^{1}\left(\left\langle c_{1}^{j}, \ldots, c_{t}^{j}\right\rangle, b_{i}^{j}\right)$,

(iv) $\left(N_{j, i},\left\{c_{1}^{j}, \ldots, c_{t}^{j}\right\},\left\{c_{1}^{j}, \ldots, c_{\lg (\bar{x})}^{j}, b_{i}^{j}\right\}, k\right)$ is simply good for $\mathfrak{K}$.

Let

$w=\left\{(i, j): i<i^{*}, j<j^{*}\right.$ and $\left(N_{j, i}, c_{1}^{j}, \ldots, c_{t}^{j}, b_{i}^{j}\right)$ is well defined $\}$.

Let

$\otimes_{6}$ like $\otimes_{5}$ but with $\oplus_{5}$ replaced by

$\oplus_{6}$ like $\oplus_{5}$ adding

(iii) for some $j,(i, j) \in w$ and $\left(B^{*}, c_{1}, \ldots, c_{t}\right) \cong\left(N_{j, i}, c_{1}^{j}, \ldots, c_{t}^{j}\right)$

Then we have

$(*)_{6} \otimes_{5} \Leftrightarrow \otimes_{6}$.

Why? By the definition of $w$.

Let

$\otimes_{7}$ there is $B$ such that: $b \in \mathcal{M}_{n}, \bar{a} \subseteq B \subseteq \mathrm{cl}^{k^{*}, m^{*}}\left(\bar{a}, \mathcal{M}_{n}\right), \operatorname{cl}^{k_{\varphi_{1}}}\left(B, \mathcal{M}_{n}\right)$ $\subseteq \mathrm{cl}^{k^{*}, m^{*}}\left(\bar{a}, \mathcal{M}_{n}\right),|B| \leq t$, and

$\oplus_{7}$ for some $c_{1}, \ldots, c_{t}$ listing $B$ such that $\bar{a}=\left\langle c_{1}, \ldots, c_{\lg (\bar{x})}\right\rangle$ there are $i<i^{*}, j<j^{*}$ such that $(i, j) \in w$ and:

(i) $\left(\mathcal{M}_{n}\left\lceil B, c_{1}, \ldots, c_{t}\right) \cong\left(N_{j}, c_{1}^{j}, \ldots, c_{t}^{j}\right)\right.$, i.e. the mapping $c_{1}^{j} \mapsto c_{1}$, $c_{2}^{j} \mapsto c_{2}$ embeds $N_{j}$ into $\mathcal{M}_{n}$

(ii) $\mathcal{M}_{n}\left\lceil\operatorname{cl}^{k_{\varphi_{1}}}\left(B, \mathcal{M}_{n}\right) \models \theta_{i}^{2}\left(\left\langle c_{1}, \ldots, c_{t}\right\rangle\right)\right.$. 
Now

$$
(*)_{7} \otimes_{6} \Leftrightarrow \otimes_{7}
$$

Why? To prove $\otimes_{6} \Rightarrow \otimes_{7}$ let $c_{1}, \ldots, c_{t}$ as well as $i<i^{*}, j<j^{*}$ be as in $\oplus_{6}$, and let $j<j^{*}$ be such that $\left(\mathcal{M}_{n}\left\lceil B, c_{1}, \ldots, c_{t}\right) \cong\left(N_{j}, c_{1}^{j}, \ldots, c_{t}^{j}\right)\right.$. The main point is that $B^{\prime}$ exemplifies that $(i, j) \in w$ (remember: $B^{\prime}$ is from $\otimes_{2}$, and if $B^{*} \in \mathcal{K}_{\infty}$, we normally could have chosen $\left.B^{\prime}=B^{*}\right)$.

To prove $\otimes_{7} \Rightarrow \otimes_{6}$ use the definition of simply good tuples in Definition $2.12(1)$.

We have now finished as $\otimes_{7}$ can be expressed as a f.o. formula straightforwardly. So we have carried out the induction step on the quantifier depth, thus finishing the proof.

(2) Similar. $\mathbf{m}_{2.17}$

CONCLUSION 2.19. (1) Assume $(\mathfrak{K}, \mathrm{cl})$ is almost nice or simply almost nice and $\mathrm{cl}$ is f.o. definable. Then: $\mathfrak{K}$ satisfies the 0-1 law iff for any $k, m$ we have

$$
(*)_{k, m}\left\langle\mathcal{M}_{n}\left\lceil\mathrm{cl}^{k, m}(\emptyset): n<\omega\right\rangle \text { satisfies the } 0-1 \text { law }\left({ }^{7}\right)\right. \text {. }
$$

(2) Similarly for the convergence law and the very weak 0-1 law.

Proof. (1) We first prove the "only if". There is a f.o. formula $\theta(x)$ such that for every random enough $\mathcal{M}_{n}, \theta(x)$ defines $\mathrm{cl}^{k, m}\left(\emptyset, \mathcal{M}_{n}\right)$. Hence for every f.o. sentence $\varphi$ there is a f.o. sentence $\psi_{\varphi}$ which is the relativization of $\varphi$ to $\theta(x)$, call it $\psi_{\varphi}$; hence, for every model $M \in \mathcal{K}, M \models \psi_{\varphi} \Leftrightarrow M \uparrow\{a$ : $M \models \theta[a]\} \mid=\varphi$. Now for every random enough $\mathcal{M}_{n}$ we have $a \in \mathcal{M}_{n} \Rightarrow$ $\mathcal{M}_{n}=\theta[a] \Leftrightarrow a \in \mathrm{cl}^{k, m}\left(\emptyset, \mathcal{M}_{n}\right)$, hence together

$$
\mathcal{M}_{n} \mid=\psi_{\varphi} \Leftrightarrow \mathcal{M}_{n}\left\lceil\mathrm{cl}^{k, m}\left(\emptyset, \mathcal{M}_{n}\right) \models \varphi .\right.
$$

As we are assuming that $\mathfrak{K}$ satisfies the 0 -1 law, for some truth value $\mathfrak{t}$ for every random enough $\mathcal{M}_{n}$,

hence (as required)

$$
\mathcal{M}_{n} \equiv " \psi_{\varphi} \equiv \mathfrak{t}
$$

$$
\mathcal{M}_{n}\left\lceil\mathrm{cl}^{k, m}\left(\emptyset, \mathcal{M}_{n}\right) \models " \varphi=\mathfrak{t} "\right.
$$

The other direction is similar by the main lemma 2.16 when $(\mathcal{K}, \mathrm{cl})$ is almost nice, 2.17 when $(\mathcal{K}, \mathrm{cl})$ is simply almost nice.

(2) Similar, so left to the reader. $\mathbf{- 2 . 1 9}$

Definition 2.20. (1) The tuple $\left(N, \bar{b}, \psi(\bar{x}),\left\langle B_{0}, B_{1}\right\rangle, k, k_{1}\right)$ is simply* good for $(\mathfrak{K}, \mathrm{cl})$ if: $B_{0}, B_{1} \leq N \in \mathcal{K}_{\infty}, \operatorname{cl}^{k}\left(B_{0}, N\right) \subseteq B_{1}, \bar{b} \in \lg (\bar{x}) N, \psi(\bar{x})$ a f.o. formula and $k, k_{1} \in \mathbb{N}$ and for every random enough $\mathcal{M}_{n}$, for every $\bar{b}^{\prime} \in$ $\lg (\bar{x})\left(\mathcal{M}_{n}\right)$ such that $\mathcal{M}_{n}\left\lceil\operatorname{cl}^{k_{1}}\left(\bar{b}^{\prime}, \mathcal{M}_{n}\right) \models \psi\left(\bar{b}^{\prime}\right)\right.$, letting $B^{\prime}=\mathcal{M}_{n}\left\lceil\operatorname{Rang}\left(\bar{b}^{\prime}\right)\right.$, there is an embedding $g$ of $N$ into $\mathcal{M}_{n}$ such that:

$\left({ }^{7}\right)$ Here we even allow empty models. 
(i) $g(\bar{b})=\bar{b}^{\prime}$,

(ii) $g(N) \cap \mathrm{cl}^{k_{1}}\left(\bar{b}^{\prime}, \mathcal{M}_{n}\right)=B^{\prime}$,

(iii) $g(N) \bigcup_{B^{\prime}} \mathrm{cl}^{k_{1}}\left(\bar{b}^{\prime}, \mathcal{M}_{n}\right)$,

(iv) $\operatorname{cl}^{k}\left(g\left(B_{0}\right), \mathcal{M}_{n}\right) \subseteq g\left(B_{1}\right) \cup \operatorname{cl}^{k_{1}}\left(B^{\prime}, \mathcal{M}_{n}\right)$.

(2) We may write $B_{0}$ instead of $\left\langle B_{0}, B_{1}\right\rangle$ if $B_{1}=N$.

(3) We say normally simply* good if (iv) is replaced by (iv) ${ }^{\prime} \mathrm{cl}^{k_{1}}\left(B^{\prime}, \mathcal{M}_{n}\right)=g\left(\mathrm{cl}^{k}\left(B_{0}, N\right)\right) \backslash B$.

Definition 2.21. The $0-1$ context with closure $(\mathfrak{K}, \mathrm{cl})$ is (normally) simply* almost nice if:

(A) for every $k, l, l^{\prime}$ there are $m^{*}=m^{*}\left(k, l, l^{\prime}\right), k^{*}=k^{*}\left(k, l, l^{\prime}\right), t=$ $t\left(k, l, l^{\prime}\right), k_{0}=k_{0}\left(k, l, l^{\prime}\right), k_{1}=k_{1}\left(k, l, l^{\prime}\right)$ such that for every random enough $\mathcal{M}_{n}$ we have: if $\bar{a} \in{ }^{l}\left|\mathcal{M}_{n}\right|$ and $b \in \mathcal{M}_{n} \backslash \mathrm{cl}^{k^{*}, m^{*}}\left(\bar{a}, \mathcal{M}_{n}\right)$ then there are $B \subseteq \mathrm{cl}^{k^{*}, m^{*}}\left(\bar{a}, \mathcal{M}_{n}\right)$ and $B^{*} \subseteq \mathcal{M}_{n}$ such that:

$(\alpha)|B| \leq t, \bar{a} \subseteq B, \mathrm{cl}^{k_{1}}\left(B, \mathcal{M}_{n}\right) \subseteq \mathrm{cl}^{k^{*}, m^{*}}\left(\bar{a}, \mathcal{M}_{n}\right)$,

( $\beta) B^{*} \supseteq B \cup\left[\mathrm{cl}^{k}\left(\bar{a} b, \mathcal{M}_{n}\right) \backslash \mathrm{cl}^{k_{1}}\left(B, \mathcal{M}_{n}\right)\right]$,

$(\gamma) B<_{s} B^{*}\left(\right.$ so $\left.B^{*} \in \mathcal{K}_{\infty}\right)$ or at least there is $B^{\prime}$ such that $B<_{s} B^{\prime}$, $\left(B^{\prime}, b, \bar{c}\right) \equiv_{l^{\prime}}\left(B^{*}, b, \bar{c}\right)$,

$(\delta) \mathcal{M}_{n}\left\lceil B^{*} \bigcup_{B^{*}}^{\mathcal{M}_{n}} \mathcal{M}_{n}\left\lceil\mathrm{cl}^{k_{1}}\left(B, \mathcal{M}_{n}\right)\right.\right.$

$(\varepsilon)$ letting $\bar{c}$ list the elements of $B$ and $\psi(\bar{x})=\bigwedge\left\{\varphi(\bar{x}): \mathcal{M}_{n}\left\lceil\mathrm{cl}^{k_{1}}\left(\bar{c}, \mathcal{M}_{n}\right) \models \varphi(\bar{x})\right.\right.$ and q.d. $\left.(\varphi(\bar{x})) \leq k_{0}\right\}$ we have: $\left(\mathcal{M}_{n}\left\lceil B^{*}, \bar{c}, \psi(\bar{x}), \bar{a} b, k, k_{1}\right)\right.$ is (normally) simply* good or at least for some $B^{\prime}, b^{\prime}$ we have:

(i) $\left(B^{\prime}, \bar{c}, \psi(\bar{x}), \bar{a} b, k, k_{1}\right)$ is (normally) simply* good,

(ii) $\left(B^{*}, b, \bar{c}\right) \equiv_{l^{\prime}}\left(B^{\prime}, b, \bar{c}\right)$.

REMARK 2.22. We may restrict $\psi$, e.g. demand that it is $\Pi_{1}$ (most natural in the cases we have).

Claim 2.23. In 2.17 we can replace simply by simply*, i.e.:

(1) Assume that $(\mathfrak{K}, \mathrm{cl})$ is simply* almost nice. Let $\varphi(\bar{x})$ be a f.o. formula. Then for some $\psi_{\varphi}(\bar{x})$ we have: for every random enough $\mathcal{M}_{n}$ and $\bar{a} \in \lg (\bar{x})\left|\mathcal{M}_{n}\right|$,

(*) $\mathcal{M}_{n} \models \varphi(\bar{a})$ if and only if $\mathcal{M}_{n}\left\lceil\mathrm{cl}^{k_{\varphi}}\left(\bar{a}, \mathcal{M}_{n}\right) \models \psi_{\varphi}(\bar{a})\right.$.

(2) We have $\left[\varphi \in \Pi_{n} \Rightarrow \psi_{\varphi} \in \Pi_{n}\right]$, $\left[\varphi \in \Sigma_{n} \Rightarrow \psi_{\varphi} \in \Sigma_{n}\right]$.

CONCLUSion 2.24. (1) Assume that $(\mathfrak{K}, \mathrm{cl})$ is (normally) simply* almost nice. Then $\mathfrak{K}$ satisfies the $0-1$ law iff $\left\langle\mathcal{M}_{n}\left\lceil\mathrm{cl}^{k, m}(\emptyset): n<\omega\right\rangle\right.$ satisfies the $0-1$ law for any $k, m$. 
(2) Assume $(\mathfrak{K}, \mathrm{cl})$ is simply* almost nice. Then $\mathfrak{K}$ satisfies the convergence law and the very weak 0-1 law iff for every $k, m,\left\langle\mathcal{M}_{n}\left\lceil\mathrm{cl}^{k, m}(\emptyset): n<\omega\right\rangle\right.$ satisfies the convergence law and the very weak 0-1 law.

3. Further abstract closure context. The context below is not used later so it can be skipped but it seems natural. In this section we are led to deal with the 0-1 law holding for monadic second order logic (i.e. we quantify over the sets). For this purpose we will use similar tools to those in $\S 2$. Looking again at Definition 2.9 or $2.12(2)(\mathrm{A})$, we note that there is an asymmetry: we try to represent $\mathrm{cl}^{k, m}\left(\bar{a} b, \mathcal{M}_{n}\right)$ and some $C \subseteq \mathrm{cl}^{k^{*}, m^{*}}\left(\bar{a}, \mathcal{M}_{n}\right)$ as free amalgamation over some $B$, small enough (with a priori bound depending on $\lg (\bar{a})$ and $k$ only, there $\left.C=\operatorname{cl}^{k}\left(B, \mathcal{M}_{n}\right)\right)$. Now this basis, $B$, of free amalgamation is included in $\mathrm{cl}^{k^{*}, m^{*}}\left(\bar{a}, \mathcal{M}_{n}\right)$ so it is without elements from $\mathrm{cl}^{k, m}\left(\bar{a} b, \mathcal{M}_{n}\right) \backslash \mathrm{cl}^{k^{*}, m^{*}}\left(\bar{a}, \mathcal{M}_{n}\right)$. Suppose we allow this and first we deal with the case where $\mathcal{M}_{n}$ is a graph. Hence a member $d$ of $\operatorname{cl}^{k, m}\left(\bar{a} b, \mathcal{M}_{n}\right)$ may code a subset of $\mathrm{cl}^{k^{*}, m^{*}}\left(\bar{a}, \mathcal{M}_{n}\right)$ : the set

$$
\left\{c \in \mathrm{cl}^{k^{*}, m^{*}}\left(\bar{a}, \mathcal{M}_{n}\right): \text { the pair }\{c, d\} \text { is an edge }\right\} .
$$

So though we are interested in f.o. formulas $\varphi(\bar{x})$ saying about $\mathcal{M}_{n}$, we are drawn into having $\psi_{\varphi}(\bar{x})$, a formula saying about $\mathrm{cl}^{k_{\varphi}, m_{\varphi}}(\bar{x})$, which is a monadic formula. Once we allow also three-place relations and more, we have to use second order logic (but still we can say which quantifiers we need because witnesses for the elimination will come from extensions of the $\left.\mathrm{cl}^{k, m}\left(\bar{a}, \mathcal{M}_{n}\right)\right)$. For this elimination, thinking of an $\mathcal{M}_{n}$, we need that any possible kind of extension of $\mathrm{cl}^{k, m}\left(\bar{a}, \mathcal{M}_{n}\right)$ occurs; so in the most natural cases, $\left|\mathrm{cl}^{k, m+1}\left(\bar{a}, \mathcal{M}_{n}\right)\right|$ may be with $2^{\left|\mathrm{cc}^{k, m}\left(\bar{a}, \mathcal{M}_{n}\right)\right|}$ elements, so in the natural case which we expect to be able to understand the situation is when there $\left|\mathrm{cl}^{k, m}\left(\bar{a}, \mathcal{M}_{n}\right)\right|<\log _{*}\left(\left|\mathcal{M}_{n}\right|\right)$. Still possibly $\mathrm{cl}^{k, m+1}\left(\bar{a}, \mathcal{M}_{n}\right)$ is not larger than $\mathrm{cl}^{k, m}\left(\bar{a}, \mathcal{M}_{n}\right)$.

However there is a big difference between the monadic case (e.g. graph where the relations coded on $\mathrm{cl}^{k^{*}, m^{*}}\left(\bar{a}, \mathcal{M}_{n}\right)$ by members of $\mathrm{cl}^{k}\left(\bar{a} b, \mathcal{M}_{n}\right)$ are monadic) and the more general case. For monadic logic addition theorems like 2.15 are known, but those are false for second order logic.

So we have good enough reason to separate the two cases. For readability we choose here to generalize the "simply almost nice with $\mathcal{K}=\mathcal{K}_{\infty}$ " case only.

Context 3.1. As in $\S 2$ for $(\mathfrak{K}, \mathrm{cl})$.

Definition 3.2. (1) The $0-1$ context with a closure operation, $(\mathfrak{K}, \mathrm{cl})$, is s.m.a. (simply monadically almost) nice if $\mathcal{K}=\mathcal{K}_{\infty}$, cl is transitive smooth local transparent (see Definitions 2.3(3), 2.5(2),(3) and 2.9(4),(5)) and:

(A) for every $k$ and $l$, there are $r=r(k, l), k^{*}=k^{*}(k, l)$ and $t_{1}=$ $t_{1}(k, l), t_{2}=t_{2}(k, l)$ such that for every $\mathcal{M}_{n}$ random enough we 
have: if $\bar{a} \in{ }^{l}\left(\mathcal{M}_{n}\right), b \in \mathcal{M}_{n}, \operatorname{cl}^{k}\left(\bar{a} b, \mathcal{M}_{n}\right) \nsubseteq \operatorname{cl}^{k^{*}}\left(\bar{a}, \mathcal{M}_{n}\right)$ then there are $B^{*}, B^{1}, B^{2}$ such that:

$(\alpha) \bar{a} \subseteq B^{1}, \operatorname{cl}^{r}\left(B^{1}, \mathcal{M}_{n}\right) \subseteq \mathrm{cl}^{k^{*}}\left(\bar{a}, \mathcal{M}_{n}\right)$ and $\left|B^{1}\right| \leq t_{1},\left|B^{2} \backslash B^{1}\right|$ $\leq t_{2}-t_{1}$

( $\beta) \bar{B}^{1} \subseteq B^{2}, B^{2} \cap \operatorname{cl}^{r}\left(B^{1}, \mathcal{M}_{n}\right)=B^{1},\left|B^{2}\right| \leq t_{2}, b \in B^{2}$,

$(\gamma) B^{*} \supseteq\left[\operatorname{cl}^{k}\left(\bar{a} b, \mathcal{M}_{n}\right) \backslash \operatorname{cl}^{r}\left(B^{1}, \mathcal{M}_{n}\right)\right] \cup B^{2}$ and $\operatorname{cl}^{k}\left(\bar{a} b, \mathcal{M}_{n}\right) \backslash$ $\operatorname{cl}^{2}\left(B^{1}, \mathcal{M}_{n}\right) \subseteq B^{*}\left(\right.$ hence $\operatorname{cl}^{k}\left(\bar{a} b, B^{*} \cup \operatorname{cl}^{2}\left(B^{1}, \mathcal{M}_{n}\right)\right)=$ $\left.\operatorname{cl}^{k}\left(\bar{a} b, \mathcal{M}_{n}\right)\right)$,

$(\delta) \mathcal{M}_{n}\left\lceil B^{*} \stackrel{\mathcal{M}_{n}}{\bigcup} \mathcal{M}_{n} \uparrow\left(B^{2} \cup \operatorname{cl}^{r}\left(B^{1}, \mathcal{M}_{n}\right)\right)\right.$ $\mathcal{M}_{n}\left\lceil B^{2}\right.$

(also here $U$ is the relation of being in free amalgamation),

$(\varepsilon)$ if $Q$ is a predicate from $\tau_{\mathcal{K}}$ and $\mathcal{M}_{n} \models Q(\bar{c}), \bar{c} \subseteq \mathrm{cl}^{r}\left(B^{1}, \mathcal{M}_{n}\right)$ $\cup B^{2}$ then $\operatorname{Rang}(\bar{c}) \cap B^{2} \subseteq B^{1}$ or $\operatorname{Rang}(\bar{c}) \backslash B^{2}$ has at most one member; if this holds we say $B^{2}$ is monadic over $\operatorname{cl}^{r}\left(B^{1}, \mathcal{M}_{n}\right)$ inside $\mathcal{M}_{n}$,

$(\zeta)\left(B^{*}, B^{1}, B^{2}, \bar{a}, b, k, r\right)$ is m.good (see below, m stands for monadically), so clearly $B^{*} \in \mathcal{K}_{\infty}$.

(2) We say $\left(B^{*}, B^{1}, B^{2}, \bar{a}, b, k\right)$ is m.good when: $B^{*}, B^{1}, B^{2} \in \mathcal{K}_{\infty}$ and $B^{1} \leq B^{2} \leq B^{*}, \bar{a} \subseteq B^{1}, b \in B^{2}$ and for every random enough $\mathcal{M}_{n}$, and $f: B^{1} \hookrightarrow \mathcal{M}_{n}$, and $C^{1} \in \mathcal{K}$ such that $\mathcal{M}_{n}\left\lceil\operatorname{cl}^{r}\left(f\left(B^{1}\right), \mathcal{M}_{n}\right) \subseteq C^{1}\right.$, and $f^{+}: B^{2} \hookrightarrow C^{1}$ extending $f$ such that $C^{1}=f^{+}\left(B^{2}\right) \cup \mathrm{cl}^{r}\left(f\left(B^{1}\right), \mathcal{M}_{n}\right)$ and $f^{+}\left(B^{2}\right)$ is monadic over $\mathrm{cl}^{r}\left(f\left(B^{1}\right), \mathcal{M}_{n}\right)$ inside $C^{1}$ (see above, but not necessarily $C^{1} \subseteq \mathcal{M}_{n}$ ) there are $g^{+}: C^{1} \hookrightarrow \mathcal{M}_{n}$ and $g: B^{*} \hookrightarrow \mathcal{M}_{n}$ such that $g\left\lceil B^{2}=\left(g^{+} \circ f^{+}\right)\left\lceil B^{2}\right.\right.$ and

$$
g\left(B^{*}\right) \underset{g\left(B^{2}\right)}{\bigcup^{+}} g^{+}\left(C^{1}\right) \quad \text { and } \quad \operatorname{cl}^{k}\left(g(\bar{a} b), \mathcal{M}_{n}\right) \subseteq g\left(B^{*}\right) \cup \operatorname{cl}^{r}\left(g\left(B^{1}\right), \mathcal{M}_{n}\right) .
$$

(3) Assume $\mathbf{E} \subseteq\left\{\left(C, B^{1}, B^{2}\right): B^{1} \leq B^{2} \leq C \in \mathcal{K}\right\}$ is closed under isomorphism. We say $B^{2}$ is E-over $D$ inside $N$ if $B^{2} \leq N \in \mathcal{K}, D \leq N$ and $\left(N \uparrow\left(B^{2} \cup D\right), B^{2} \cap D, B^{2}\right) \in \mathbf{E}$.

(4) We say $\left(B^{*}, B^{1}, B^{2}, \bar{a}, b, k, r\right)$ is $\mathbf{E}$ - $\operatorname{good}$ when $B^{*}, B^{1}, B^{2} \in \mathcal{K}_{\infty}$ and $B^{1} \leq B^{2} \leq B^{*}, \bar{a} \subseteq B^{1}, b \in B^{2}$ and for every random enough $\mathcal{M}_{n}$ and $f: B^{1} \hookrightarrow C^{1} \in \mathcal{K}$ extending $f$ such that $C^{1}=f^{+}\left(B^{2}\right) \cup \mathrm{cl}^{r}\left(f\left(B^{1}\right), \mathcal{M}_{n}\right)$ and $f^{+}\left(B^{2}\right)$ is E-over $\operatorname{cl}^{r}\left(f\left(B^{1}\right), \mathcal{M}_{n}\right)$ inside $C^{1}$ (see above but not necessarily $\left.C^{1} \subseteq \mathcal{M}_{n}\right)$ there are $g^{+}: C^{1} \hookrightarrow \mathcal{M}_{n}$ and $g: B^{*} \hookrightarrow \mathcal{M}_{n}$ such that $g\left\lceil B^{2}=\left(g^{+} \circ f^{+}\right)\left\lceil B^{2}\right.\right.$ and $g\left(B^{*}\right) \quad \cup g^{+}\left(C^{1}\right)$ and $\operatorname{cl}^{k}\left(g(\bar{a} b), \mathcal{M}_{n}\right) \subseteq$ $g\left(B^{*}\right) \cup \mathrm{cl}^{r}\left(g\left(B^{1}\right), \mathcal{M}_{n}\right)$. $g\left(B^{2}\right)$

(5) We say $\mathfrak{K}$ is $s . \mathbf{E} . a$. nice if in 3.2(1) we replace clauses $(\varepsilon),(\zeta)$ by

$(\varepsilon)^{\prime} B^{2}$ is $\mathbf{E}$-over $\operatorname{cl}^{r}\left(B^{1}, \mathcal{M}_{n}\right)$ inside $\mathcal{M}_{n}$,

$(\zeta)^{\prime}\left(B^{*}, B^{1}, B^{2}, \bar{a}, b, k, r\right)$ is $\mathbf{E}$-good. 
(6) We say $\mathbf{E}$ is monadic if it is as in part (3) and $\left(C, B^{1}, B^{2}\right) \in \mathbf{E}$ implies

$$
\bar{a} \in Q^{C} \Rightarrow\left(\operatorname{Rang}(\bar{a}) \cap B^{2} \subseteq B^{1}\right) \vee\left(\left|\operatorname{Rang}(\bar{a}) \backslash B^{2}\right| \leq 1\right) .
$$

(7) We say $\mathbf{E}$ as in 3.2(3) is simply monadic if it is monadic and for any $B^{1} \leq B^{2} \in \mathcal{K}$, letting $\Gamma_{B^{2}}=\left\{\theta(y, \bar{b}): \bar{b} \subseteq B^{2}\right.$ is with no repetition, $\theta(y, \bar{x})$ is a basic formula, each variable actually appearing $\}$

we have: the class $\left\{\left(D, R_{\theta(y, \bar{b})}, c\right)_{\theta(y, \bar{b}) \in \Gamma_{B^{2}}, c \in B^{1}}: D \in \mathcal{K}, B^{1} \leq D, R_{\theta(y, \bar{b})}\right.$ is a subset of $D \backslash B^{1}$, there are $C^{1}, f$ such that: $\left(C^{1}, f\left(B^{1}\right), f\left(B^{2}\right)\right)$ $\in \mathbf{E}, D \leq C^{1} \in \mathcal{K}, f: B^{2} \hookrightarrow C^{1}, f\left(B^{2}\right) \cap D=$ $B^{1}, f\left\lceil B^{1}=\operatorname{id}_{B^{1}}\right.$, and for $\theta(y, \bar{b}) \in \Gamma_{B^{2}}$ we have $\left.R_{\theta(y, \bar{b})}=\left\{d \in D \backslash B^{1}: C^{1}=\theta[d, f(\bar{b})]\right\}\right\}$

is definable by a monadic formula $\left({ }^{8}\right)$.

(8) We say that cl is monadically definable for $\mathcal{K}$ if for each $k$, letting $\bar{x}=$ $\left\langle x_{l}: l<k\right\rangle$, for some monadic formula $\Theta_{k}(y, x)$ we have: $y \in \operatorname{cl}^{k}\left(\bar{x}, \mathcal{M}_{n}\right) \Leftrightarrow$ $\mathcal{M}_{n}\left\lceil\operatorname{cl}^{k}\left(\bar{x}, \mathcal{M}_{n}\right) \models \Theta_{k}(\bar{x}, y)\right.$ holds for every random enough $\mathcal{M}_{n}$.

(9) We say that $\mathbf{E}$ is trivial if it is $\left\{\left(C, B^{1}, B^{2}\right): C \cup B^{2}, B^{1} \leq B^{2} \leq C\right\}$. $B^{1}$

Lemma 3.3. Assume $(\mathfrak{K}, \mathrm{cl})$ is s.E.a. nice and $\mathbf{E}$ is simply monadic and $\mathrm{cl}$ is f.o. definable or at least monadically definable (see 3.2(7)). Then for every f.o. formula $\varphi(\bar{x})$ there are $k$ and a monadic formula $\psi_{\varphi}(\bar{x})$ such that:

$(*)_{\varphi(\bar{x})}$ for every random enough $\mathcal{M}_{n}$, for every $\bar{a} \in \lg (\bar{x})\left|\mathcal{M}_{n}\right|$ we have

$$
\mathcal{M}_{n}=\varphi(\bar{a}) \Leftrightarrow \mathcal{M}_{n}\left\lceil\operatorname{cl}^{k}\left(\bar{a}, \mathcal{M}_{n}\right) \models \psi_{\varphi}(\bar{a})\right.
$$

Discussion 3.4. Some of the assumptions of 3.3 are open to manipulations; others are essential.

(1) As said above, the "monadic" is needed in order to use an addition theorem (see 3.5), the price of removing it is high: essentially above we need that after finding the copy $g\left(B^{2}\right)$ realizing the required type over $\mathrm{cl}^{k}\left(B^{1}, \mathcal{M}_{n}\right)$, we need to find $g\left(B^{*}\right)$, or a replacement like $B^{\prime}$ in the proofs in $\S 2$ but only the validity of some formula $\varphi(\ldots, b, \ldots)_{b \in B^{2}}$ in $B^{*}$ is important. Now what if the requirements on the type of $g\left(B^{2}\right)$ over $\mathrm{cl}^{r}\left(B^{1}, \mathcal{M}_{n}\right)$ are not coded by some subsets of $\mathrm{cl}^{r}\left(B^{1}, \mathcal{M}_{n}\right)$ but e.g. by two-place relations on $\mathrm{cl}^{r}\left(B^{1}, \mathcal{M}_{n}\right)$ ? So naturally we allow quantification over two-place relations in the formulas $\psi_{\varphi}(\bar{x})$. But then even though

$\left({ }^{8}\right)$ We can restrict ourselves to the cases $C=\mathrm{cl}^{k}(B, C)$. 


$$
B^{*} \bigcup_{B^{2}} \operatorname{cl}^{r}\left(B^{1}, \mathcal{M}_{n}\right) \cup B^{2}
$$

not only the small formulas satisfied by $\left(B^{2}, b\right)_{b \in B^{1}}$ are important but also e.g. the answer to $B^{*} \cong \mathrm{cl}^{r}\left(B^{1}, \mathcal{M}_{n}\right)$.

It is natural to demand that all possibilities for the set of small formulas in second order logic satisfied by $B^{*} \cup \mathrm{cl}^{r}\left(B^{1}, \mathcal{M}_{n}\right)$ occur so this may include cases where $B^{*}$ has to be of cardinality much larger than $\mathrm{cl}^{k}\left(\bar{a}, \mathcal{M}_{n}\right)$. So we do not formulate such a lemma. Of course some specific information may help to control the situation. We may however consider adding (in 3.2) the demand:

$\bigotimes_{s}$ if $Y \subseteq B^{*} \cup \mathrm{cl}^{r}\left(B^{1}, \mathcal{M}_{n}\right)$ and $Y \cap B^{*} \nsubseteq B^{2}, Y \cap \mathrm{cl}^{r}\left(B^{1}, \mathcal{M}_{n}\right) \nsubseteq B^{1}$, then $Y$ is not $s$-connected, that is, for some $Y_{1}, Y_{2}$, we have $Y=$ $Y_{1} \cup Y_{2},\left|Y_{1} \cap Y_{2}\right| \leq s$ and

$$
Y_{1} \underset{Y_{1} \cap Y_{2}}{\bigcup_{2}} \quad \text { (i.e. } \mathcal{M}_{n}\left\lceil Y_{1} \underset{\mathcal{M}_{n}\left\lceil Y_{1} \cap Y_{2}\right.}{\bigcup} \mathcal{M}_{n}\left\lceil Y_{2}\right) .\right.
$$

In this case we can allow e.g. quantification on two-place relations $R$ such that $\mathcal{M}_{n}\lceil\operatorname{Dom}(R)$ is not $s$-connected.

(2) If $\mathbf{E}$ is monadic but not simply monadic, not much is changed: we should allow new quantifiers in $\psi_{\varphi}$. Let $C^{1}<_{B}^{\mathbf{E}} C^{2}$ if $B \leq C^{1} \leq$ $C^{2}$ and $\left(C^{2}, B, B \cup\left(C^{2} \backslash C^{1}\right)\right) \in \mathbf{E}$. We want the quantifier to say for $\left(C^{1}, R_{\theta(y, \bar{b})}, c\right)_{\theta(\bar{y}, \bar{b}) \in \Gamma, c \in B}$ that it codes $C^{2}$ with $C^{1} \leq \underset{B}{\mathbf{E}} C^{2}$ where $\Gamma=$ $\Gamma_{B \cup\left(C^{2} \backslash C^{1}\right)}$, but then the logic should be defined such that we would be able to iterate.

The situation is similar to the case where in $\S 2$, we have: cl is definable or at least monadically definable.

(3) In 3.3 we essentially demand

(*) for each $t$, for random enough $\mathcal{M}_{n}$, for every $B \subseteq \mathcal{M}_{n}$ with $|B|$ $\leq t$, if $\mathcal{M}_{n}\left\lceil\operatorname{cl}^{k}\left(B, \mathcal{M}_{n}\right)<_{B}^{\mathbf{E}} C\right.$ then $C$ is embeddable into $\mathcal{M}_{n}$ over $\operatorname{cl}^{k}\left(B, \mathcal{M}_{n}\right)$.

Of course we need this just for a dense set of such $C^{\prime}$ 's, dense in the sense that a monadic sentence is satisfied, just like the use of $B^{\prime}$ in 2.12. That is, we may replace clause (3) of Definition 3.2(1)(A) by

$(\zeta)^{\prime}$ there is $B^{\prime}$ such that $\left(B^{\prime}, c, b\right)_{c \in B_{2}} \equiv_{l}^{\prime}\left(B^{*}, c, b\right)_{c \in B_{2}}$ and $\left(B^{\prime}, B^{1}, B^{2}\right.$, $\bar{a}, k$ ) is m. good (and $l^{\prime}$ large enough, e.g. quantifier depth of $\psi_{\varphi_{1}}$ in the main case).

(4) As we have done in 2.16(2), 2.17(2), we can add that the number of alternations of quantifiers of $\varphi$ and the number of (possible) alternations of monadic quantifiers of $\psi_{\varphi}$ are equal as long as the depth of the formulas from "simply monadic" is not counted. (Always we can trivially increase 
the q.d. so we may ask about $\psi_{\varphi}$ with minimal number.) But for a specific $\left\langle\mathcal{M}_{n}: n<\omega\right\rangle$ we may get better. We can though look at minimal q.d. on all cases then it should be trivial.

(5) Can we find a reasonable context where the situation from 3.3 and 3.4(1) above holds? Suppose we draw edges as here in $\mathcal{M}_{n}^{0}$ and redraw in the neighbourhood of each edge. Let us describe the drawing fully, for a model on $[n]$. For each $i<j$ from $[n]$ we flip a coin $\mathcal{E}_{i, j}$ on whether we have $(i, j)$ as a pre-edge, with probability $p_{i, j}^{n}$. If we succeed for $\mathcal{E}_{i, j}$ then for any pair $\left(i^{\prime}, j^{\prime}\right)$ from $[n]$ we flip a coin $\mathcal{E}_{i, j, i^{\prime}, j^{\prime}}$ with probability $p_{i, j, i^{\prime}, j^{\prime}}^{n}$. The flippings are independent and finally for $i^{\prime}<j^{\prime},\left(i^{\prime}, j^{\prime}\right)$ is an edge if and only if for some $i<j,(i, j)$ is a pre-edge, that is, we succeed in $\mathcal{E}_{i, j}$ and we also succeed in $\mathcal{E}_{i, j, i^{\prime}, j^{\prime}}$. For our case let $\left(\alpha \in(0,1)_{\mathbb{R}}\right.$ is irrational):

\section{Distribution 1.}

$$
p_{i, j}^{n}=p_{|i-j|}= \begin{cases}1 /|i-j|^{\alpha} & \text { when }|i-j|>1, \\ 1 / 2^{\alpha} & \text { if }|i-j|=1\end{cases}
$$

and $p_{i, j, i^{\prime}, j^{\prime}}^{n}=1 / 2^{\left|i-i^{\prime}\right|+\left|j-j^{\prime}\right|}$.

Distribution 2. $p_{i, j}^{n}$ is as above and

$$
p_{i, j, i^{\prime}, j^{\prime}}^{n}= \begin{cases}1 / 2^{\left|i-i^{\prime}\right|+\left|j-j^{\prime}\right|} & \text { if } i=i^{\prime} \vee j=j^{\prime}, \\ 0 & \text { otherwise. }\end{cases}
$$

Now distribution 2 seems to give us an example as in Lemma 3.3, distribution 1 fits the nonmonadic case. Distribution 1 will give us, for some pre-edges $(i, j)$, a lot of edges in their neighbourhood; of course for the average pre-edge there will be few. This gives us a lot of $\leq_{i}$ extensions in that neighbourhood. We may wonder whether actually the $0-1$ law holds. It seems to me intuitively clear that for distribution 2 the answer is "yes", for distribution 1 the answer is "no".

(6) Why we think that in distribution 1 from (5) the 0-1 law should fail (in fact fails badly)? It seems to me that for distribution 1 we can find $A \subseteq B$ such that for every random enough $\mathcal{M}_{n}$, for some $f: A \hookrightarrow \mathcal{M}_{n}$, the number of $g: B \hookrightarrow \mathcal{M}_{n}$ extending $f$ is quite large, and on the set of such $g$ we can interpret an initial segment $N_{f}$ of arithmetic even with $f(A)$ a segment, $N_{f}$ in its neighbourhood. The problem is to compare such $N_{f_{1}}, N_{f_{2}}$ with possibly distinct parameters, which can be done using a path of pre-edges from $f_{1}(A)$ to $f_{2}(A)$. But this requires further thoughts.

The case of distribution 2 should be similar to this paper.

We intend to return to this.

(7) If $\mathbf{E}$ is trivial, then the claim above becomes (a variant of) the main claim in Section 2 (the variant fulfils promises there). 
Proof of 3.3. This proof is similar to that of Lemmas 2.16 and 2.17. We say in the claim that $\psi_{\varphi}(\bar{x})$ or $\psi_{\varphi}(\bar{x}), k_{\varphi}$ witness $(*)_{\varphi(\bar{x})}$. We prove the statement by induction on q.d. $(\varphi(\bar{x}))$ and first note (by clause (d) of Definition 2.2) that $(*)_{\varphi(\bar{x})} \Rightarrow(*)_{\varphi(\bar{x})}^{+}$where $\psi_{\varphi}(\bar{x})$ will be monadic logic and

$(*)_{\varphi(\bar{x})}^{+}$for every random enough $\mathcal{M}_{n}$, for every $\bar{a} \in \lg (\bar{x})\left(\mathcal{M}_{n}\right)$ and $N$, if $\mathcal{M}_{n}\left\lceil\operatorname{cl}^{k \varphi}\left(\bar{a}, \mathcal{M}_{n}\right) \subseteq N \subseteq \mathcal{M}_{n}\right.$ then $\mathcal{M}_{n}=\varphi[\bar{a}] \Leftrightarrow N \models \psi_{\varphi}[\bar{a}]$.

Case 1: $\varphi(\bar{x})$ an atomic formula. Trivial.

Case 2: $\varphi(\bar{x})$ a Boolean combination of atomic formulas and formulas $\varphi(\bar{x})$ of the form $(\exists y) \varphi^{\prime}(\bar{x}, y), \varphi^{\prime}$ of quantifier depth $<r$, such that $(*)_{(\exists y) \varphi^{\prime}(\bar{x}, y)}$ holds. Clearly follows by cases 3 and 1 .

Case 3: $\varphi(\bar{x})=(\exists y) \varphi_{1}(\bar{x}, y)$. Let $k_{\varphi_{1}}, \psi_{\varphi_{1}}$ witness $(*)_{\varphi(\bar{x})}$ of 3.3 and let $k_{\varphi_{1}} \psi_{\varphi_{1}}^{2}$ witness that $(*)_{\varphi_{1}(\bar{x})}^{+}$holds for it (for $\left.\varphi_{1}\right)$. Let $r=r\left(k_{\varphi_{1}}, \lg (\bar{x})\right)$, $k^{*}=k^{*}\left(k_{\varphi_{1}}, \lg (\bar{x})\right), t_{1}=t_{1}(k, \lg (\bar{x}))$ and $t_{2}=t_{2}(k, \lg (\bar{x}))$ be as in Definition $3.2(1)(\mathrm{A})$, more exactly its $3.2(3)$ variant. Let $k_{\varphi}$ be $k^{*}$.

It is enough to prove the following two statements:

StATEMENT 1. There is a monadic formula $\psi_{\varphi}^{1}(\bar{x})$ such that:

$(*)_{1}$ for every random enough $\mathcal{M}_{n}$ and for every $\bar{a} \in \lg (\bar{x})\left|\mathcal{M}_{n}\right|$ we have $(\alpha)_{1} \Leftrightarrow(\beta)_{1}$ where:

$(\alpha)_{1} \mathcal{M}_{n}\left\lceil\mathrm{Cl}^{k^{*}}\left(\bar{a}, \mathcal{M}_{n}\right) \models \psi_{\varphi}^{1}(\bar{a})\right.$,

$(\beta)_{1} \mathcal{M}_{n} \models$ "there is b satisfying $\mathrm{cl}^{k_{\varphi_{1}}}\left(\bar{a} b, \mathcal{M}_{n}\right) \subseteq \mathrm{cl}^{k^{*}}\left(\bar{a}, \mathcal{M}_{n}\right)$ such that $\varphi_{1}(\bar{a}, b)$ holds."

Statement 2. There is a monadic formula $\psi_{\varphi}^{2}(\bar{x})$ such that:

$(*)_{2}$ for every random enough $\mathcal{M}_{n}$ and for every $\bar{a} \in \lg (\bar{x})\left|\mathcal{M}_{n}\right|$ we have $(\alpha)_{2} \Leftrightarrow(\beta)_{2}$ where:

$(\alpha)_{2} \mathcal{M}_{n}\left\lceil\mathrm{cl}^{k^{*}}\left(\bar{a}, \mathcal{M}_{n}\right) \models \psi_{\varphi}^{2}(\bar{a})\right.$,

$(\beta)_{2} \mathcal{M}_{n} \models$ "there is b satisfying $\mathrm{cl}^{k_{\varphi_{1}}}\left(\bar{a} b, \mathcal{M}_{n}\right) \nsubseteq \mathrm{cl}^{k^{*}}\left(\bar{a}, \mathcal{M}_{n}\right)$ such that $\varphi_{1}(\bar{a}, b)$ holds."

(Note: $(\beta)_{1},(\beta)_{2}$ are complementary, but it is enough that always at least one holds.)

Note that as " $y \in \mathrm{cl}^{k^{*}}(\bar{x})$ " is monadically definable, by 3.2(7)(d) and by the choice of $k_{\varphi}$ we can in $(\alpha)_{2}$ replace $\mathrm{cl}^{k^{*}}$ by $\mathrm{cl}^{k_{\varphi}}$, changing $\psi_{\varphi}^{2}$ to $\psi_{\varphi}^{2.5}$, and similarly in $(\alpha)_{1}$ replace $\mathrm{cl}^{k^{*}}$ by $\mathrm{cl}^{k_{\varphi}^{*}}$ changing $\psi_{\varphi}^{1}$ to $\psi_{\varphi}^{1.5}$. quired.

Clearly these two statements are enough and $\psi_{\varphi}^{1.5}(\bar{x}) \vee \psi_{\varphi}^{2.5}(\bar{x})$ is as re-

Proof of Statement 1. Easy, by the induction hypothesis and by the fact that the closure is sufficiently definable. 
Proof of Statement 2. We will use a series of equivalent statements $\otimes_{l}$.

$\otimes_{1}$ is $(\beta)_{2}$.

$\otimes_{2}$ There are $b, B$ and $B^{*}, B_{1}, B_{2}$ such that: $b \in \mathcal{M}_{n}, \operatorname{cl}^{k_{\varphi_{1}}}\left(\bar{a} b, \mathcal{M}_{n}\right) \nsubseteq$ $\mathrm{cl}^{k^{*}}\left(\bar{a}, \mathcal{M}_{n}\right), \bar{a} \subseteq B_{1} \subseteq \mathrm{cl}^{k^{*}}\left(\bar{a}, \mathcal{M}_{n}\right), \operatorname{cl}^{r}\left(B_{1}, \mathcal{M}_{n}\right) \subseteq \mathrm{cl}^{k^{*}}\left(\bar{a}, \mathcal{M}_{n}\right)$, $\left|B_{1}\right| \leq t_{1},\left|B_{2}\right| \leq t_{2},\left|B_{2} \backslash B_{1}\right| \leq t_{2}-t_{1}, B_{1} \leq B_{2} \leq B^{*}, b \in B_{2}$, $B^{*} \backslash B_{1}$ is disjoint from $\operatorname{cl}^{r}\left(B_{1}, \mathcal{M}_{n}\right)$ and $\left({ }^{9}\right) B_{1} \leq_{s} B^{*} \in \mathcal{K}_{\infty}$ and

$$
B^{*} \bigcup_{B_{2}}^{\mathcal{M}_{n}} B_{2} \cup \mathrm{cl}^{r}\left(B_{1}, \mathcal{M}_{n}\right)
$$

and $\operatorname{cl}^{k_{\varphi_{1}}}\left(\bar{a} b, \mathcal{M}_{n}\right) \backslash \mathrm{cl}^{2}\left(B_{1}, \mathcal{M}_{n}\right) \subseteq B^{*}$ (therefore we have $\operatorname{cl}^{k_{\varphi_{1}}}(\bar{a} b$, $\left.\left.B^{*} \cup \operatorname{cl}^{2}\left(B_{1}, \mathcal{M}_{n}\right)\right)=\operatorname{cl}^{k_{\varphi_{1}}}\left(\bar{a} b, \mathcal{M}_{n}\right)\right)$ and $\left(B^{*}, B_{1}, B_{2}, \bar{a}, b, k, r\right)$ is Egood, and

$\oplus_{2} \mathcal{M}_{n} \models \varphi_{1}(\bar{a}, b)$.

We have

$$
(*)_{2} \otimes_{1} \Leftrightarrow \otimes_{2} .
$$

Why? The implication $\Leftarrow$ is trivial, the implication $\Rightarrow$ holds by clause (A) in Definition 3.2.

Next, let

$\otimes_{3}$ like $\otimes_{2}$ but with $\oplus_{2}$ replaced by

$$
\oplus_{3} \mathcal{M}_{n}\left\lceil\mathrm{cl}^{k_{\varphi_{1}}}\left(\bar{a} b, M_{n}\right) \models \psi_{\varphi_{1}}(\bar{a}, b) .\right.
$$

Then

$$
(*)_{3} \otimes_{2} \Leftrightarrow \otimes_{3} .
$$

Why? By the induction hypothesis, i.e. choice of $k_{\varphi_{1}}, \psi_{\varphi_{1}}$.

Next, let

$\otimes_{4}$ like $\otimes_{3}$ but with $\oplus_{3}$ replaced by

$$
\oplus_{4} \mathcal{M}_{n} \uparrow\left[B^{*} \cup \operatorname{cl}^{r}\left(B_{1}, \mathcal{M}_{n}\right)\right] \models \psi_{\varphi_{1}}^{2}(\bar{a}, b) .
$$

Then

$$
(*)_{4} \otimes_{3} \Leftrightarrow \otimes_{4} .
$$

Why? By $(*)_{\varphi_{1}}^{+}$being witnessed by $\psi_{\varphi_{1}}^{2}, k_{\varphi_{1}}$ (see the beginning of the proof), the definition of $B^{*}$ and the choice of $\psi_{\varphi_{1}}^{2}$.

For notational simplicity we assume $B \neq \emptyset$, and similarly assume $\bar{a}$ is with no repetition and apply Lemma 3.5 below with the vocabulary $\tau_{\mathcal{K}}$ to the case $s=t_{2}, \bar{z}^{2}$ empty, $\bar{z}^{1}=\left\langle z_{1}^{1}\right\rangle, \bar{z}=\left\langle z_{1}, \ldots, z_{t_{2}}\right\rangle$, and $\psi\left(\bar{z}, \bar{z}^{1}, \bar{z}^{2}\right)=$ $\psi\left(\bar{z}, z_{1}^{1}\right)=\psi_{\varphi}^{2}\left(\left\langle z_{1}, \ldots, z_{\lg (\bar{x})}\right\rangle, z_{1}^{1}\right)$ and get $i^{*}, \theta_{i}^{1}\left(\bar{z}, \bar{z}^{1}\right)$ and $\theta_{i}^{2}(\bar{z})$ for $i<i^{*}$ as there.

$\left({ }^{9}\right)$ The $B^{\prime}$ does not appear for simplicity of exposition only. 
Next, let

$\otimes_{5}$ like $\otimes_{4}$ but with $\oplus_{4}$ replaced by

$\oplus_{5}$ letting $c_{1}, \ldots, c_{t_{2}}$ list $B_{2}$ possibly with repetitions but such that $\left\{c_{1}, \ldots, c_{t_{1}}\right\}=B_{1}$ and $\left\langle c_{1}, \ldots, c_{\lg (\bar{x})}\right\rangle=\bar{a}$, there is $i<i^{*}$ such that:

(i) $B^{*} \models \theta_{i}^{1}\left[\left\langle c_{1}, \ldots, c_{t_{2}}\right\rangle, b\right]$,

(ii) $\mathcal{M}_{n} \uparrow\left(B_{2} \cup \mathrm{cl}^{k}\left(B_{1}, \mathcal{M}_{n}\right)\right) \models \theta_{i}^{2}\left[\left\langle c_{1}, \ldots, c_{t_{2}}\right\rangle\right]$.

Now

$(*)_{5} \otimes_{4} \Leftrightarrow \otimes_{5}$.

Why? By the choice of $\theta_{i}^{1}, \theta_{i}^{2}\left(i<i^{*}\right)$.

Let $\mathcal{P}=\left\{\left(N, c_{1}, \ldots, c_{t_{2}}\right): N \in \mathcal{K}_{\infty}\right.$, with the set of elements $\left.\left\{c_{1}, \ldots, c_{t_{2}}\right\}\right\}$. Let $\left\{\left(N_{j}, c_{1}^{j}, \ldots, c_{t_{2}}^{j}\right): j<j^{*}\right\}$ list the members of $\mathcal{P}$ up to isomorphism, so with no two isomorphic. For every $j<j^{*}$ and $i<i^{*}$ choose if possible $\left(N_{j, i}, c_{1}^{j}, \ldots, c_{t_{2}}^{j}, b_{i}^{j}\right)$ such that:

(i) $N_{j} \leq_{s} N_{j, i}\left(\right.$ in $\left.\mathcal{K}_{\infty}\right)$,

(ii) $b_{i}^{j} \in N_{j, i} \backslash N_{j}$,

(iii) $N_{j, i}=\theta_{i}^{1}\left(\left\langle c_{1}^{j}, \ldots, c_{t_{2}}^{j}\right\rangle, b_{i}^{j}\right)$,

(iv) $\left(N_{j, i},\left\{c_{1}^{j}, \ldots, c_{t_{1}}^{j}\right\},\left\{c_{1}^{j}, \ldots, c_{t_{2}}^{j}\right\},\left\{c_{1}^{j}, \ldots, c_{\lg (\bar{x})}^{j}, b_{i}^{j}\right\}, k\right)$ is E-good.

Let

$$
w=\left\{(i, j): i<i^{*}, j<j^{*} \text { and }\left(N_{j, i}, c_{1}^{j}, \ldots, c_{t}^{j}, b_{i}^{j}\right) \text { is well defined }\right\} .
$$

Let $\Gamma=\left\{\theta(y, \bar{x}): \theta\right.$ is a basic formula, $\left.\bar{x} \subseteq\left\{x_{1}, \ldots, x_{t_{2}}\right\}\right\}$.

As $\mathbf{E}$ is simply monadic (see Definition 3.2(7)) we have: for some monadic formula $\theta_{i}^{3}$, if $\left\{d_{1}, \ldots, d_{t_{1}}\right\} \leq C \in \mathcal{K}$ and $\Gamma:=\left\{\theta\left(y, \ldots, x_{i(l)}, \ldots\right)_{l<l(*)}: \theta\right.$ an atomic formula for $\tau_{\mathcal{K}}$, every variable actually appears and $\left.i(l) \in\left\{1, \ldots, t_{2}\right\}\right\}$, then the following are equivalent:

(a) There are subsets $R_{\theta}$ of $C$ for $\theta \in \Gamma$ and there are $C_{1}, d_{t}\left(t=t_{1}+1\right.$, $\left.\ldots, t_{2}\right)$ satisfying $R_{\varphi(y, \bar{x})} \subseteq C$ and $C \leq C_{1} \in \mathcal{K}, C_{1} \backslash C=\left\{d_{t_{1}+1}, \ldots, d_{t_{2}}\right\}$, and

$$
R_{\theta\left(y, \ldots, x_{i}, \ldots\right)}=\left\{e \in C: C_{1} \models \theta\left[e, \ldots, d_{i}, \ldots\right]\right\} \quad \text { for } \theta\left(y, \ldots, x_{i}, \ldots\right) \in \Gamma
$$

and $C_{1}=\theta_{i}^{2}\left[d_{1}, \ldots, d_{t_{2}}\right]$.

(b) $C \models \theta_{i}^{3}\left[d_{1}, \ldots, d_{t_{1}}\right]$.

Let

$\otimes_{6}$ There are $b, B_{1}$ such that: $b \in \mathcal{M}_{n}, \operatorname{cl}^{k_{\varphi_{1}}}\left(\bar{a} b, \mathcal{M}_{n}\right) \nsubseteq \operatorname{cl}^{k^{*}}\left(\bar{a}, \mathcal{M}_{n}\right), \bar{a} \subseteq$ $B_{1} \subseteq \operatorname{cl}^{k^{*}}\left(\bar{a}, \mathcal{M}_{n}\right), \operatorname{cl}^{r}\left(B_{1}, \mathcal{M}_{n}\right) \subseteq \operatorname{cl}^{k^{*}}\left(\bar{a}, \mathcal{M}_{n}\right),|B| \leq t_{1}\left(k_{\varphi_{1}}, \lg (\bar{x})\right)$, and 
$\otimes_{6}$ for some $c_{1}, \ldots, c_{t_{1}}$ listing $B_{1}$ such that $\bar{a}=\left\langle c_{1}, \ldots, c_{\lg (\bar{x})}\right\rangle$ there are $i<i^{*}, j<j^{*}$ such that:

(i) $\left(\mathcal{M}_{n}\left\lceil B^{1}, c_{1}, \ldots, c_{t_{1}}\right) \cong\left(N_{j}, c_{1}^{j}, \ldots, c_{t_{1}}^{j}\right)\right.$, i.e. the mapping $c_{1}^{j} \mapsto c_{1}, c_{2}^{j} \mapsto c_{2}$ embeds $N_{j}$ into $\mathcal{M}_{n}$

(ii) $\mathcal{M}_{n}\left\lceil\mathrm{cl}^{r}\left(B^{1}, \mathcal{M}_{n}\right) \models \theta_{i}^{3}\left(\left\langle c_{1}, \ldots, c_{t_{1}}\right\rangle\right)\right.$.

Then

$$
(*)_{6} \otimes_{5} \Leftrightarrow \otimes_{6} .
$$

Why? To prove $\otimes_{5} \Rightarrow \otimes_{6}$ let $c_{1}, \ldots, c_{t_{2}}$ as well as $i<i^{*}$ be as in $\oplus_{5}$, and let $j<j^{*}$ be such that $\left(\mathcal{M}_{n}\left\lceil B^{1}, c_{1}, \ldots, c_{t_{1}}\right) \cong\left(N_{j}, c_{1}^{j}, \ldots, c_{t_{1}}^{j}\right)\right.$. A main point is that $B^{*}$ exemplifies that $(i, j) \in w$.

To prove $\otimes_{6} \Rightarrow \otimes_{5}$ use part (B) of Definition 2.9.

Now we have finished as $\otimes_{6}$ can be expressed as a monadic formula straightforwardly. So we have carried out the induction step on the quantifier depth, thus finishing the proof. $\mathbf{- 3 . 3}$

The following is the parallel of 2.15 for monadic logic (see Gurevich [Gu], more [Sh 463]).

LEMMA 3.5. For a finite vocabulary $\tau$ and a monadic formula (in the vocabulary $\tau) \psi\left(\bar{z}, \bar{z}^{1}, \bar{z}^{2}\right), \bar{z}=\left\langle z_{1}, \ldots, z_{s}\right\rangle$, there are $i^{*} \in \mathbb{N}$ and monadic $\tau$-formulas $\theta_{i}^{1}\left(\bar{z}, \bar{z}^{1}\right)=\theta_{i, \psi}^{1}\left(\bar{z}, \bar{z}^{1}\right), \theta_{i}^{2}(\bar{x}, \bar{z})=\theta_{i, \psi}^{2}\left(\bar{z}, \bar{z}^{2}\right)$ for $i<i^{*}$, each of quantifier depth at most that of $\psi$, such that if

$$
N_{1} \underset{N_{0}}{N} N_{2}, \quad N_{1} \cap N_{2}=N_{0}, \quad N_{1} \cup N_{2}=N
$$

and the set of elements of $N_{0}$ is $\left\{c_{1}, \ldots, c_{s}\right\}, \bar{c}=\left\langle c_{1}, \ldots, c_{s}\right\rangle$ and $\bar{c}^{1} \in$ $\lg \left(\bar{z}^{1}\right)\left(N_{1}\right)$ and $\bar{c}^{2} \in \lg \left(\bar{z}^{2}\right)\left(N_{2}\right)$, then

$$
N \models \psi\left[\bar{c}, \bar{c}^{1}, \bar{c}^{2}\right] \quad \text { iff } \quad \text { for some } i<i^{*}, N_{1} \models \theta_{i}^{1}\left[\bar{c}, \bar{c}^{1}\right] \text { and } N_{2}=\theta_{i}^{2}\left[\bar{c}, \bar{c}^{2}\right] \text {. }
$$

\section{References}

[B196] J. T. Baldwin, Near model completeness and 0-1 laws, preprint, 1996.

[BlSh 528] J. Baldwin and S. Shelah, Randomness and semigenericity, Trans. Amer. Math. Soc. 349 (1997), 1359-1376; math.LO/9607226 $\left({ }^{10}\right)$.

[BoSp] R. B. Boppana and J. Spencer, Smoothness laws for random ordered graphs, in: Logic and Random Structures (New Brunswick, NJ, 1995), DIMACS Ser. Discrete Math. Theoret. Comput. Sci. 33, Amer. Math. Soc., Providence, RI, 1997, 15-32.

[CK] C. C. Chang and J. H. Keisler, Model Theory, Stud. Logic Found. Math. 73, North-Holland, Amsterdam, 1973.

$\left({ }^{10}\right)$ References of the form math.XX/... refer to the xxx.lanl.gov archive. 
[Gu] Y. Gurevich, Monadic second-order theories, in: J. Barwise and S. Feferman (eds.), Model Theoretic Logics, Chap. XIII, Springer, New York, 1985, 479506.

[LuSh 435] T. Łuczak and S. Shelah, Convergence in homogeneous random graphs, Random Structures Algorithms 6 (1995), 371-391; math.LO/9501221.

[Sh 463] S. Shelah, On the very weak 0-1 law for random graphs with orders, J. Logic Comput. 6 (1996), 137-159; math.LO/9507221.

[Sh 517] - Zero-one laws for graphs with edge probabilities decaying with distance. Part II, Fund. Math., submitted.

[Sh 548] - Very weak zero one law for random graphs with order and random binary functions, Random Structures Algorithms 9 (1996), 351-358; math.LO/ 9606230.

[Sh 550] -, 0-1 laws, preprint; math.LO/9804154.

[Sh 581] - When 0-1 law holds for $G_{n, \bar{p}}, \bar{p}$ monotonic, in preparation.

[Sh 637] -, 0-1 laws: Putting together two contexts randomly, in preparation.

[Sh:F192] - Lecture notes on 0-1 laws, October'95, Rutgers.

[ShSp 304] S. Shelah and J. Spencer, Zero-one laws for sparse random graphs, J. Amer. Math. Soc. 1 (1988), 97-115.

[Sp] J. Spencer, Zero-one laws with variable probability, J. Symbolic Logic 58 (1993), 1-14.

Institute of Mathematics

The Hebrew University of Jerusalem 91904 Jerusalem, Israel

E-mail: shelah@math.huji.ac.il
Department of Mathematics Rutgers University New Brunswick, NJ 08854, U.S.A. URL: http://www.math.rutgers.edu/ shelah

Received 19 July 1996;

in revised form 26 June 2002 